\title{
Hydrodynamic Modeling of Towed Buoyant Submarine Antenna's in Multidirectional Seas
}

\author{
by \\ Sam R. Geiger \\ B.S., Louisiana Tech University, 1993 \\ Submitted to the Department of Ocean Engineering, MIT \\ and to the Department of Applied Ocean Physics and Engineering, WHOI \\ in partial fulfillment of the requirements for the degree of \\ Master of Science in Oceanographic Engineering \\ at the \\ MASSACHUSETTS INSTITUTE OF TECHNOLOGY \\ and the \\ WOODS HOLE OCEANOGRAPHIC INSTITUTION
}

September 2000

(C) 2000 Sam R. Geiger

All rights reserved.

The author hereby grants to MIT and WHOI permission to reproduce paper and electronic copies of this thesis in whole or in part and to distribute them publicly.

Signature of Author

Joint Program in Oceanographic Engineering

Massachusetts Institute of Technology

and Woods Hole Oceanographic Institution

August 4, 2000

Certified by

Jerome H. Milgram

Professor, Ocean Engineering

Thesis Supervisor

Accepted by

Professor MichaeY S. Triantafyllou

Chair, Joint Committee for Oceanographic Engineering

Massachusetts Institute of Technology and Woods Hole Oceanographic Institution 


\title{
Hydrodynamic Modeling of Towed Buoyant Submarine Antenna's in Multidirectional Seas \\ by
}

Sam R. Geiger

\begin{abstract}
Submitted to the Department of Ocean Engineering, MIT and to the Department of Applied Ocean Physics and Engineering, WHOI on August 4, 2000 in partial fulfillment of the requirements for the degree of Master of Science in Oceanographic Engineering
\end{abstract}

\begin{abstract}
A finite difference computer model is developed to simulate the exposure statistics of a radio frequency buoyant antenna as it is towed in a three-dimensional random seaway. The model allows the user to prescribe antenna properties (length, diameter, density, etc.), sea conditions (significant wave height, development of sea), tow angle, and tow speed. The model then simulates the antenna-sea interaction for the desired duration to collect statistics relating to antenna performance. The model provides design engineers with a tool to predict antenna performance trends, and to conduct design tradeoff studies. The floating antenna envisioned is for use by a submarine operating at modest speed and depth.
\end{abstract}

Thesis Supervisor: Jerome H. Milgram

Title: Professor, Ocean Engineering 


\section{Contents}

1 Introduction $\quad 9$

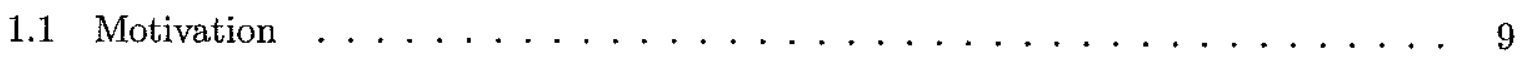

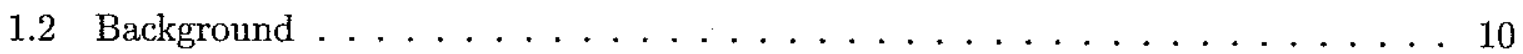

1.3 Antenna Model . . . . . . . . . . . . . . . . . . . 11

2 Wave Field Model Development $\quad 12$

2.1 Linear, Random, Deep Water Wave Theory . . . . . . . . . . . . . . 12

2.2 Energy Density Spectra Contained in the Model . . . . . . . . . . . . 16

2.2.1 Bretschneider Two Parameter Spectrum . . . . . . . . . . . . 18

2.2 .2 Ochi Six Parameter Spectrum . . . . . . . . . . . . . 20

2.2 .3 Square Wave Spectrum . . . . . . . . . . . . . . 22

2.2 .4 Single Sine Wave Spectrum . . . . . . . . . . . . . . 22

2.3 Unidirectional Modeling Methods . . . . . . . . . . . . . . 23

2.3.1 Sine Wave Superposition (SWS) Method . . . . . . . . . . . . . 24

2.3.2 Fast Fourier Transform (FFT) Method . . . . . . . . . . . . . 25

2.4 Multidirectional Modeling Methods . . . . . . . . . . . . . . . 28

2.4.1 Complete Wave Field (CWF) Method . . . . . . . . . . . . . . 29

2.4 .2 Partial Wave Field (PWF) Method . . . . . . . . . . . . . . . . 34

2.5 Verification of Model Seas . . . . . . . . . . . . . . . 43

2.5.1 Significant Wave Height . . . . . . . . . . . . . . 43

2.5 .2 Encounter Frequency . . . . . . . . . . . . . . 45

2.5 .3 Spatial Spectral Analysis . . . . . . . . . . . . . . 46 
2.5.4 Temporal Spectral Analysis . . . . . . . . . . . . . . . 49

3 Antenna Model Development $\quad 51$

3.1 Coordinate System Description . . . . . . . . . . . . . . 51

3.2 Governing Vertical Equation of Motion . . . . . . . . . . . . 53

3.3 Antenna Modeling Methods . . . . . . . . . . . . . . . . 55

3.3 .1 Discretization . . . . . . . . . . . . . . . . 55

3.3 .2 Integration Method $\ldots \ldots \ldots \ldots \ldots \ldots$

3.3 .3 Boundary Conditions $\ldots \ldots \ldots \ldots \ldots$

3.3 .4 Initial Conditions . . . . . . . . . . . . . . . . 59

4 Modeling Results $\quad 60$

4.1 Exposure Statistics . . . . . . . . . . . . . . . . 6 60

4.1 Definition of Exposure . . . . . . . . . . . . 60

4.1.2 Statistics Calculated by the Model . . . . . . . . . . . . . 61

4.2 Results . . . . . . . . . . . . . . . . . . . 63

4.2.1 Series 1: Statistics vs. Number of Propagation Directions . . . . . . . 63

4.2 .2 Series 2: Statistics vs. Tow Angle . . . . . . . . . . . . 65

4.2 .3 Series 3: Statistics vs. Sea Severity . . . . . . . . . . . . 67

4.2 .4 Series 4: Statistics vs. Tow Speed . . . . . . . . . . . . . . . 69

4.2.5 Series 5: Statistics vs. Antenna Length . . . . . . . . . . . . 71

4.2.6 Series 6: Statistics vs. Antenna Diameter . . . . . . . . . . . . 73

4.2.7 Series 7: Statistics vs. Antenna Density . . . . . . . . . . . . . 75

$\begin{array}{lll}5 & \text { Future Efforts } & 77\end{array}$

$\begin{array}{ll}\text { A User's Guide to Proteus } & 78\end{array}$

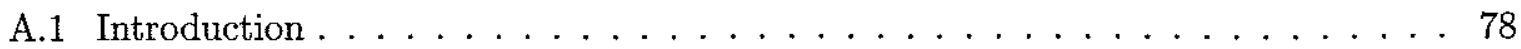

A.1.1 Contents of the User's Guide . . . . . . . . . . . . . 78

A.1.2 Analysis Procedure . . . . . . . . . . . . . 78

A.2 Proteus Input File . . . . . . . . . . . . . . . . 81

A.2.1 Description of Features and Limitations . . . . . . . . . . . 81 
A.2.2 Sample Input File $\ldots \ldots \ldots \ldots$. . . . . . . . . . . . . . .

A.3 Output Files $\ldots \ldots \ldots \ldots \ldots \ldots \ldots \ldots \ldots \ldots \ldots \ldots \ldots \ldots \ldots$

A.3.1 Snapshot Output File $\left(r u n_{-} i d / /{ }^{\prime}\right.$ mat'$\left.^{\prime}\right) \ldots \ldots \ldots$. . . . . . . 91

A.3.2 Simulation Parameters and Results File (run_id//'_info.txt') . . . . . 92

A.3.3 Exposure Data Matrix File (run_id//'_edm') . . . . . . . . . . 95

A.3.4 Temporal Wave Data File $\left(r u n_{-} i d / /{ }^{\prime}{ }_{\text {mat2' }}\right) \ldots \ldots \ldots$. . . . . . 95

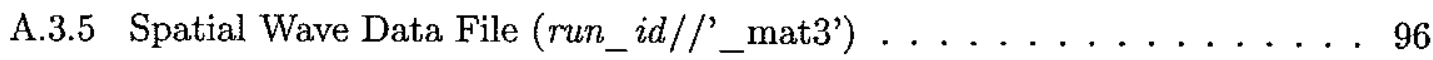

$\begin{array}{ll}\text { B Sea State Correlation Data } & 98\end{array}$ 


\section{List of Figures}

2-1 Typical Wave Energy Density Spectrum . . . . . . . . . . . . . . . . 14

2-2 Determination of Component Amplitudes $a_{i}(\omega) \ldots \ldots \ldots \ldots$

2-3 Bretschneider Spectrum for Varying Sea Severity . . . . . . . . . . . 18

2-4 Bretschneider Spectrum for Varying Degrees of Development $\ldots \ldots \ldots \ldots$

2-5 Ochi Six Parameter Single Most Probable Spectrum . . . . . . . . . . . . . . 21

$2-6$ Square Wave Spectrum . . . . . . . . . . . . . . . . . 22

$2-7$ Single Sine Wave Spectrum . . . . . . . . . . . . . 23

2-8 Complete Wave Field 3-D Energy Spectrum (quadrants 1 and 4 shown) . . . . 31

2-9 Wave Field Generated by CWF Method . . . . . . . . . . . . . . . . . 34

2-10 PWF Unidirectional Wave Summation . . . . . . . . . . . . . 35

2-11 Partial Wave Field 3-D Energy Spectrum - No Directional Scaling (quadrants 1

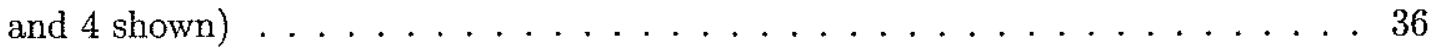

$2-12$ Cosine Square Directional Spreading Function . . . . . . . . . . 38

2-13 Cosine Square Directional Spreading Function for the Five Directions Example Problem . . . . . . . . . . . . . . . . . . . . 38

2-14 Partial Wave Field 3-D Energy Spectrum - With Directional Scaling (quadrants

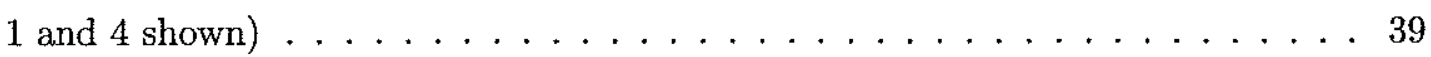

$2-15$ Geometry of the Wave Summation . . . . . . . . . . . . . . 41

2-16 Encounter Frequency for Varying Tow Speeds . . . . . . . . . . . . . . . 45

2-17 Spectral Analysis of Spatial Wave Data - One Propagation Direction . . . . . . 46

2-18 Spectral Analysis of Spatial Wave Data - Three Propagation Direcţions . . . . 47

2-19 Spectral Analysis of Spatial Wave Data - Five Propagation Directions . . . . . 48 
2-20 Spatial Frequency Stretching . . . . . . . . . . . . . . 49

2-21 Spectral Analysis of Temporal Wave Data . . . . . . . . . . . . . 50

3-1 Coordinate System: X-Z Plane . . . . . . . . . . . . . . . 52

$3-2$ Coordinate System: X-Y Plane . . . . . . . . . . . . . . . . 52

3-3 Antenna and Sea Surface Elevation Variables . . . . . . . . . . . . 54

3-4 Antenna Element Description . . . . . . . . . . . . . . . . . . 55

3-5 Antenna and Antenna Element Description . . . . . . . . . . . 56

4-1 Average Exposure vs. Number of Propagation Directions . . . . . . . . . . . 64

$4-2$ Average Exposure vs. Tow Angle . . . . . . . . . . . . . . . . . 66

43 Average Exposure vs. Sea Severity . . . . . . . . . . . . . . . 68

$4-4$ Average Exposure vs. Tow Speed . . . . . . . . . . . . . . . . 70

$4-5$ Average Exposure vs. Antenna Length . . . . . . . . . . . . . . . . . 72

$4-6$ Average Exposure vs. Antenna Diameter . . . . . . . . . . . . . . . 74

$4-7$ Average Exposure vs. Antenna Density . . . . . . . . . . . . . . 76

A-1 Sample Threshold Antenna Exposure of $0.04 \ldots \ldots$. . . . . . . . . 89

A-2 Sample Output of Snapshot Output File . . . . . . . . . . . . . . . 92

A-3 Sample Output of Temporal Wave Data Analysis . . . . . . . . . . . . . 96

A-4 Sample Output of Spatial Wave Data Analysis . . . . . . . . . . . . . 97 


\section{List of Tables}

2.1 Linear Theory Deep Water Wave Relations . . . . . . . . . . . . 13

2.2 Ochi Six Parameter Spectrum: Most Probable Values . . . . . . . . . . . 21

2.3 Comparison of Fourier Transform Domain Relationships . . . . . . . . . . . 25

2.4 Comparison of Frequency Resolution for the SWS and FFT Methods . . . . . . . 28

2.5 Simulated Significant Wave Heights for a Specified Sea Severity of $3.0 \mathrm{~m}$. . . . 44

4.1 Series 1 Input Parameters . . . . . . . . . . . . . . . . 63

4.2 Series 2 Input Parameters . . . . . . . . . . . . . . . . 65

4.3 Series 3 Input Parameters . . . . . . . . . . . . . . . . . 67

4.4 Series 4 Input Parameters . . . . . . . . . . . . . . . . . . . 69

4.5 Series 5 Input Parameters . . . . . . . . . . . . . . 71

4.6 Series 6 Input Parameters . . . . . . . . . . . . . . . . . 73

4.7 Series 7 Input Parameters . . . . . . . . . . . . . . 75

B.1 Sea State Correlation Data . . . . . . . . . . . . . . . . . 99 


\section{Chapter 1}

\section{Introduction}

\subsection{Motivation}

As the United States Navy launches the last of the highly capable Seawolf class submarines and begins construction on the even more capable Virginia class submarines, it continues to find itself operationally limited by the technological barriers present with high data rate communications. The capability for high speed data transfer has escalated at an astonishing rate over the last few years; however, the submarine force faces unique challenges in fully utilizing high data rate systems which operate at higher frequencies. High frequency (UHF, SHF, or EHF band) radio signals are unable to penetrate below the surface of the water; so, while the submarine is submerged, radio communications systems are limited to low data rate receive-only modes transmitted at very low frequencies (ELF, or VLF band). The current mode of operation calls for the submarine to routinely come to periscope depth to raise a radio mast above the surface of the water, send and receive the necessary message traffic, then submerge to continue its mission. This required visit to periscope depth not only causes the submarine to suspend execution of its mission, but also reduces the overall stealth of the mission. Submarine operations are typically driven by the need for stealth, and stealth is best preserved by remaining fully submerged. Clearly, the communications goal of the submarine force is to be able to utilize the high frequency and high data rate communication circuits while retaining the stealth of operation at speed and depth. 


\subsection{Background}

This goal of high data rate communication at speed and depth is the motivation of a U.S. Navy Advanced Technology Demonstration (ATD). Initially funded jointly by the Defense Advanced Research Projects Agency (DARPA) and the Office of Naval Research (ONR) in FY 96-97 as a feasibility study, the Buoyant Cable Array Antenna (BCAA) research became a DARPA and ONR Demonstration in FY 98-99. The BCAA prototype satisfactorily demonstrated receiveonly capability with a field test in the Summer of 1999 . The program was promoted to the level of Navy ATD in FY 00 and renamed the Multi-element Buoyant Cable Antenna (MBCA). The MBCA is a 3 year joint program which is a combined Navy ATD-funded and DARPA-funded effort. This ATD, one of only 6 ATD's funded by the Navy for fiscal year 2000, reflects the high priority the Navy places on submarine connectivity. The goals of the MBCA program include:

- demonstrate 24 kbps 2-way communication at UHF over the Fleet Satellite (FLTSAT) communications system while the submarine is at a speed of 6 knots and at a depth of 300 feet

- demonstrate 1.5 Mbps data rates over UHF Line-of-Site

- demonstrate L-band voice communications.

The participants in the program include the Naval Undersea Warfare Center, Johns Hopkins University Applied Physics Laboratory, Sippican Inc., and MIT's Lincoln Laboratory. Lincoln Laboratory is a federally funded laboratory administered by the Massachusetts Institute of Technology. Lincoln Laboratory, whose specialties include radar and electrical engineering, was chosen to overcome the technological challenge of designing a floating linear array radio frequency communication antenna module and the associated inboard electronics. However, to gain insight into the behavior of the antenna module in a random seaway, Lincoln Laboratory added Professor Jerome Milgram of the MIT Ocean Engineering department to the antenna development effort. Professor Milgram joined a working group that was established to estimate statistics on

- the average fraction of antenna elements exposed over an extended period of time 
- the temporal variation of the exposure statistics, including duration of reception dropout times

- the spatial distribution of those exposed elements.

This thesis work was undertaken to support the statistics working group by improving upon the first hydrodynamic model which was developed by Professor Milgram and Gary Ulrich. [9][10] The improved model retains all of the capabilities of the original model and improves upon the original model by upgrading the wave field from a unidirectional wave field with the choice of only two tow directions, 0 or 180 degrees, to a multidirectional wave field with the choice of any tow direction from 0 to 180 degrees. This provides all angles due to port and starboard symmetry.

\subsection{Antenna Model}

The method used to determine the antenna behavior and the exposure statistics was to develop long term simulations from which exposure statistics could be obtained vice getting the exposure statistics from the theory of a non-linear process. The model, named PROTEUS, performs the simulation by constructing a wave field and then modeling the antenna in the wave field and analyzing the resultant behavior. The development of the wave field model is described in Chapter 2 and the development of the antenna model is described in Chapter 3 . The results of the improved model are presented in Chapter 4 and a detailed user's guide is included as Appendix A. 


\section{Chapter 2}

\section{Wave Field Model Development}

\subsection{Linear, Random, Deep Water Wave Theory}

To simplify the model and to reduce computation time, the simulated wave field was assumed to be described by linear, random, deep-water wave theory. The linear theory aspect of the waves implies that the velocity potential and fluid velocity are constant from the mean free-surface to the free-surface level. The linear theory assumptions represent a first order approximation in satisfying the free surface kinematic and dynamic boundary conditions. A complete derivation and discussion of linear wave theory can be found in Faltinsen and Newman. [4][6] "Deep water" is defined to exist when $\lambda<2 d$, where $\lambda$ is the wavelength and $d$ is the water depth. The random aspect of the waves implies that

- the instantaneous wave elevation from the mean free-surface is gaussian distributed with zero mean and variance

- the waves are an ergodic process, which means that the wave statistics obtained from a single location are the same as the wave statistics obtained from an ensemble of locations

- the waves are a stationary process, which means that the wave statistics are invariant with respect to time [7][8]

Because the last condition is so restrictive, we relax it to allow the statistics to vary slowly with time, otherwise known as a weakly-stationary process. A summary of the deep water linear theory wave relations is provided in Table 2.1. 


\begin{tabular}{ll}
\hline Quantity & Formula \\
\hline \hline Wave Number & $k=\frac{2 \pi}{\lambda}$ \\
Circular Frequency & $\omega=\frac{2 \pi}{T}$ \\
Dispersion Relation & $k=\frac{\omega^{2}}{g}$ \\
Velocity Potential & $\phi=\frac{a g}{\omega} e^{k z} \sin \left(k x^{\prime}-\omega t\right)$ \\
Surface Displacement & $h=a \cos \left(k x^{\prime}-\omega t\right)$ \\
Radian Frequency & $\omega=\sqrt{g k}$ \\
Phase Velocity & $c=\sqrt{g / k}$ \\
Group Velocity & $c_{g}=\frac{1}{2} \sqrt{g / k}$ \\
Horizontal Fluid Velocity & $u=a \omega e^{k z} \cos \left(k x^{\prime}-\omega t\right)$ \\
Vertical Fluid Velocity & $w=a \omega e^{k z} \sin \left(k x^{\prime}-\omega t\right)$ \\
Wave Energy Density & $E=\frac{1}{2} \rho g a^{2}$ \\
& \\
Note: $x^{\prime}$ refers to a stationary x coordinate system.
\end{tabular}

Table 2.1: Linear Theory Deep Water Wave Relations

Assuming a lower wave frequency bound of $0.3 \mathrm{rad} / \mathrm{sec}$, which is reasonable for the vast majority of energy density spectra one would encounter, this translates into a minimum water depth of 342 meters. This implies that for depths shallower than about 350 meters, the deep water theory begins to break down, and a finite depth treatment of the waves must be considered. A complete derivation and discussion of finite depth wave theory can be found in Faltinsen and Newman. [4][6]

Upon inspection of the linear theory formulas in Table 2.1, the waves are found to be sinusoidal. However, an observation of the sea surface shows that the waves display a quite complicated and random structure. This observed behavior can be numerically approximated by adding together a series of sinusoids with each discrete frequency component having the appropriate amplitude and a random phase. As the number of discrete frequency components increases, the numerical approximation approaches the continuous distribution of the actual sea surface. This discrete method of describing a two dimensional random seaway is presented in Equation 2.1

$$
h\left(x^{\prime}, t\right)=\sum_{i=1}^{N} a_{i}(\omega) \cos \left(k_{i}(\omega) x^{\prime}-\omega_{i} t+\phi_{i}\right)
$$

where 


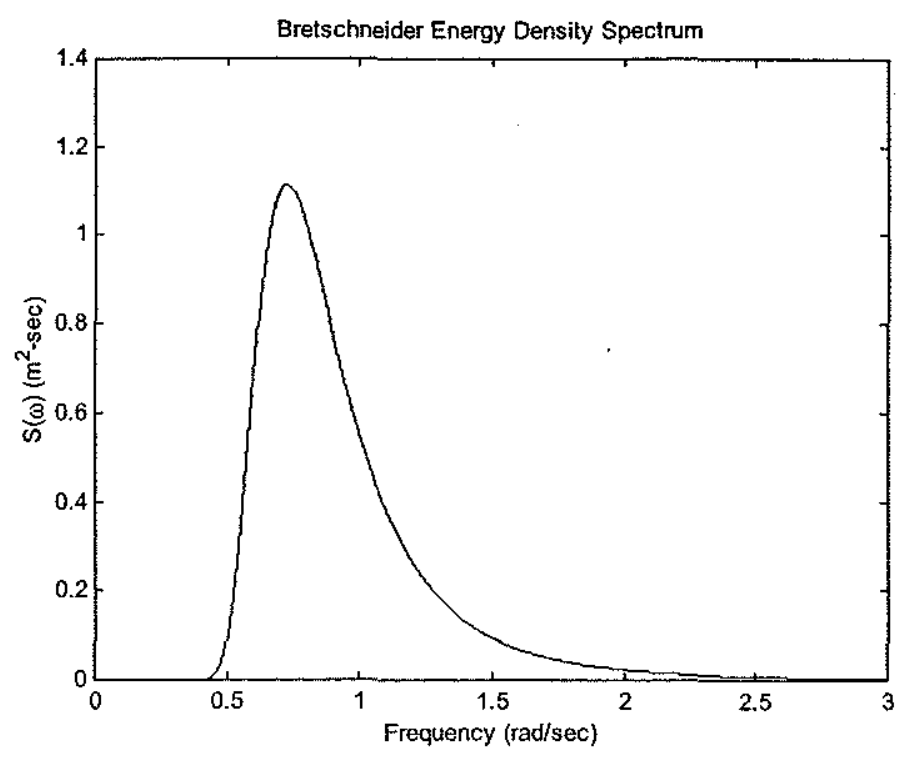

Figure 2-1: Typical Wave Energy Density Spectrum

$h\left(x^{\prime}, t\right)$ is the wave elevation, referenced to the mean free-surface

$a_{i}(\omega)$ is the appropriate wave amplitude of each discrete frequency component

$k_{i}(\omega)$ is the wave number of each discrete frequency component

$\omega_{i}$ is the set of discrete circular frequencies contained in the modeled energy density spectrum

$\phi_{i}$ is the random phase assigned to each discrete frequency component

and the $e^{k z}$ term has been dropped, since evaluation occurs at the sea surface $(z=0)$.

Using the equations found in Table 2.1, one can determine most of the terms on the right hand side of Equation 2.1: $k_{i}$ is determined from the dispersion relation, and $\phi_{i}$ is a random number with a uniform distribution on 0 to $2 \pi$. The determination of the appropriate wave amplitude, however, requires some discussion and a deeper understanding of wave theory.

To determine the wave amplitude corresponding to a given wave frequency, one begins by defining the spectral energy density function, $S(\omega)$, which represents the energy of the waves having frequency $\omega$. A typical energy density spectrum is shown in Figure 2-1. The units of $S(\omega)$ are defined to be in $m^{2}-s e c$, so that when $S(\omega)$ is integrated with respect to $\omega$, the result is the average energy of the random waves with respect to time. 


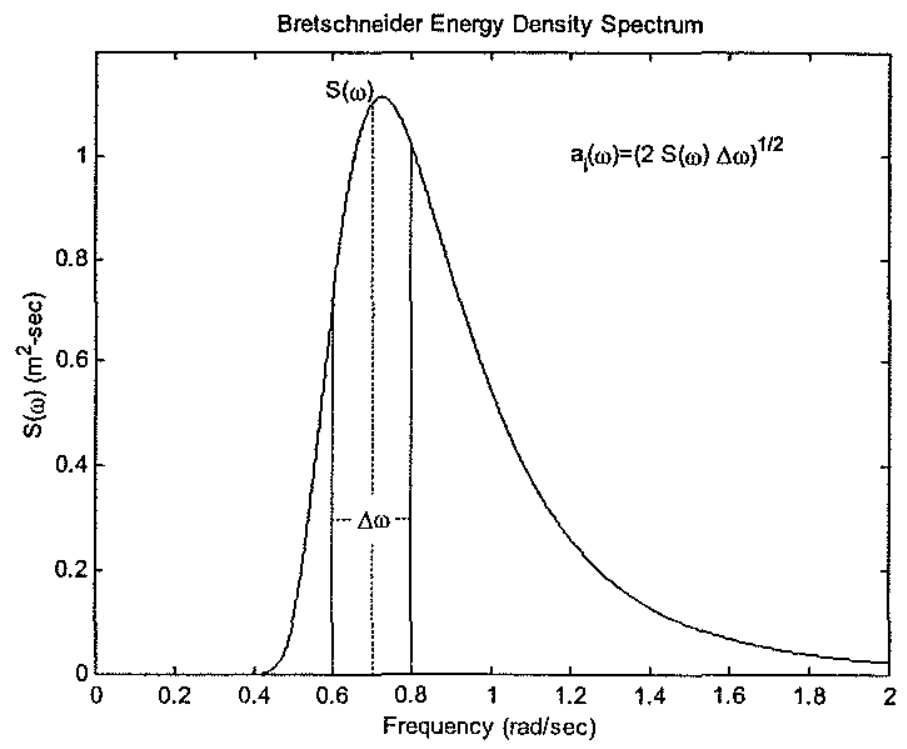

Figure 2-2: Determination of Component Amplitudes $a_{i}(\omega)$

The spectrum shown in Figure 2-1 is said to be a single-sided spectrum, which refers to the fact that the frequencies are constrained to be positive. For a double-sided spectrum, both the positive and the negative frequencies are allowed, and the spectrum is defined such that $S(\omega)=S(-\omega)$. In addition, the double-sided spectrum is half the magnitude of the single-sided spectrum; since, for constant sea severity, the area under the spectral energy density curve must be constant. The amplitudes, $a_{i}(\omega)$, of the discrete frequency components in Equation 2.1 are obtained from Equation 2.2

$$
\frac{1}{2} a_{i}(\omega)^{2}=S(\omega) \Delta \omega
$$

where

$a_{i}(\omega)$ is the amplitude of the discrete band, $\Delta \omega$, of waves with center frequency $\omega$.

Equation 2.2 is simply a statement that the square of the wave amplitude is a measure of the average energy of the waves, because both sides of the equation represent energy in units of $m^{2}$. The method of obtaining the amplitudes $a_{i}(\omega)$ is shown in Figure 2:2. 
Until now, $S(\omega)$ has been presented in terms of wave energy present at a given wave frequency. It can be shown from the Wiener-Khintchine theorem that $S(\omega)$ is related to the wave amplitude record at a fixed location in the ocean. [7] We define a wave record at a given location as $x^{\prime}(t)$. Then the autocorrelation of $x^{\prime}(t)$ is given by

$$
R(\tau)=\lim _{T \rightarrow \infty} \frac{1}{2 T} \int_{-T}^{T} x^{\prime}(t) x^{\prime}(t+\tau) d t
$$

and by the Wiener-Khintchine theorem, the spectral energy density function, $S(\omega)$, and the autocorrelation function, $R(\tau)$, form a Fourier transform pair.

$$
\begin{aligned}
& S(\omega)=\frac{1}{\pi} \int_{-\infty}^{\infty} R(\tau) e^{-i \omega \tau} d \tau \\
& R(\tau)=\frac{1}{2} \int_{-\infty}^{\infty} S(\omega) e^{-i \omega \tau} d \omega
\end{aligned}
$$

This method proves to be invaluable as it has the ability to convert observed wave records into spectral energy density functions.

\subsection{Energy Density Spectra Contained in the Model}

Numerous energy density spectral forms have been proposed by researchers attempting to characterize a particular sea wave environment. [4][5][7][8] The spectral forms may characterize a particular geographic wave environment or they may characterize a particular storm environment, but in all cases, they share a very similar form. The model incorporates the spectral formulation as a subroutine; therefore, it is completely arbitrary from the model viewpoint how the spectrum is defined. In order to provide some variability in the allowable spectral forms and to allow for troubleshooting and debugging of the model, four wave spectral energy forms were included in the model. Two of the spectral forms are actual derived models that are well accepted in the ocean design field. Though the two spectral models may appear very different at first glance, most spectral formulations share the basic form of Equation 2.6 [9]

$$
S(\omega)=\frac{A}{\omega^{5}} H_{s}^{2} e^{-B / \omega^{4}}
$$


where

$A, B$ are constants to be determined

$H_{s}$ is the significant wave height, which is defined to be the average wave height of the one third highest waves. Sea severity is most commonly given in terms of significant wave height among researchers; however, significant data exists which gives sea severity in terms of a value called sea state. Appendix B provides a correlation amongst significant wave height, modal wave frequency, wind speed, and sea state. The two actual derived spectral formulations chosen for the model are the Bretschneider two parameter spectrum and the Ochi six parameter spectrum, given in Equations 2.7 and 2.8, respectively. [7]

$$
\begin{gathered}
S(\omega)=\frac{1.25}{4} \frac{\omega_{m}^{4}}{\omega^{5}} H_{s}^{2} e^{-1.25\left(\omega_{m} / \omega\right)^{4}} \\
S(\omega)=\frac{1}{4} \sum_{j=1}^{2} \frac{\left(\frac{4 \lambda_{j}+1}{4} \omega_{m j}^{4}\right)^{\lambda_{j}}}{\Gamma\left(\lambda_{j}\right)} \frac{H_{s j}^{2}}{\omega^{4 \lambda_{j}+1}} e^{-\left(\frac{4 \lambda_{j}+1}{4}\right)\left(\omega_{m j} / \omega\right)^{4}}
\end{gathered}
$$

The other two models are the square wave spectrum and the single sine wave spectrum, given in Equations 2.9 and 2.10, respectively. These simple geometric form models were used for troubleshooting and debugging of the model and to verify the results of the model.

$$
\begin{gathered}
S(b)=\left\{\begin{array}{ccc}
0.0 & \text { for } & b<0.01 \\
5.0 & \text { for } & 0.01 \leq b \leq 1.01 \\
0.0 & \text { for } & b>1.01
\end{array}\right. \\
S(b)=5 \delta\left(b-b_{s}\right)
\end{gathered}
$$

where 


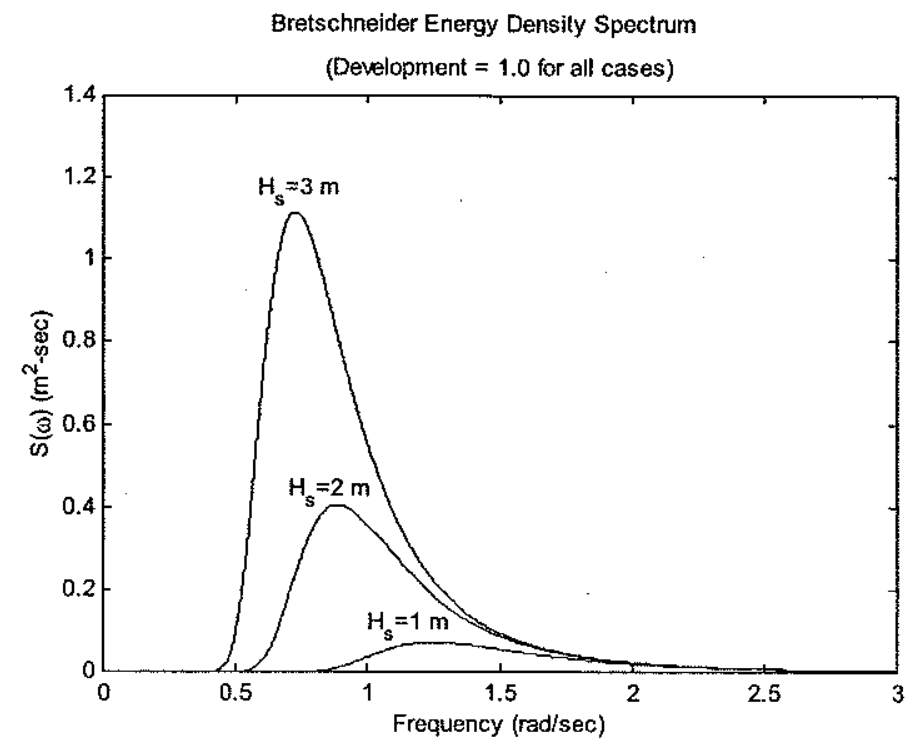

Figure 2-3: Bretschneider Spectrum for Varying Sea Severity

$$
b=\frac{k}{2 \pi}
$$

and $b_{s}$ is the spatial frequency of the sine wave.

\subsubsection{Bretschneider Two Parameter Spectrum}

The two parameters of the Bretschneider spectrum, Equation 2.7, are significant wave height, $H_{s}$, and modal frequency, $\omega_{m}$. Modal frequency is the frequency at which $S(\omega)$ reaches its maximum value, and significant wave height is as previously defined. For the special case of a fully developed sea, a relationship exists between $\omega_{m}$ and $H_{s}$. A fully developed sea, also referred to as a fully arisen sea, is one in which the wind imparts energy to the waves at a rate equal to that at which viscous damping dissipates their energy and wave-wave interactions transmit energy from one frequency to another. Designating this modal frequency as $\omega_{m_{F A}}$, we 


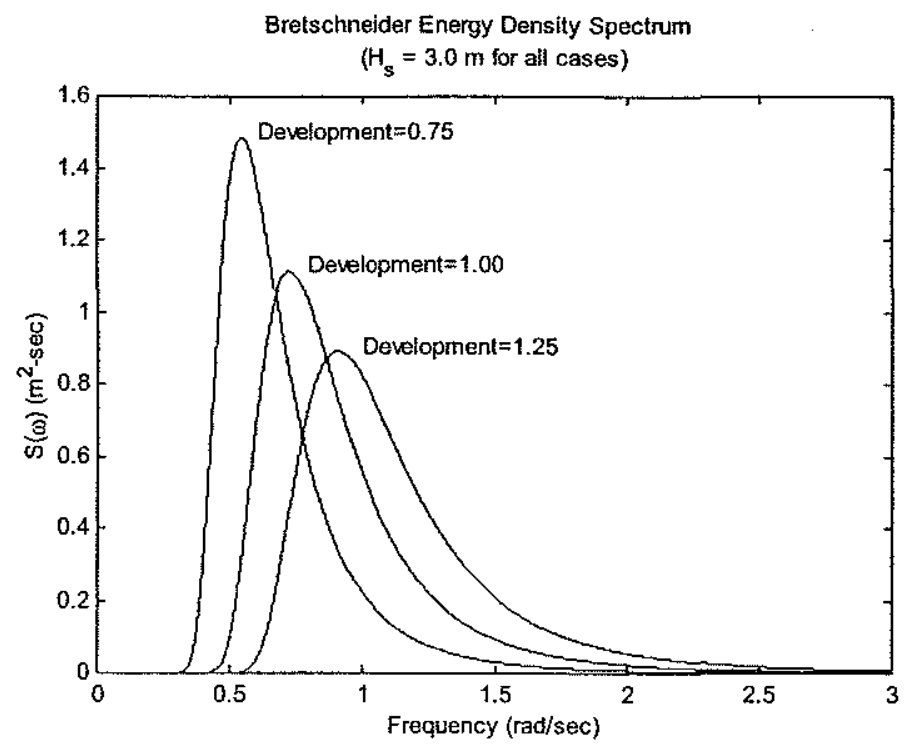

Figure 2-4: Bretschneider Spectrum for Varying Degrees of Development

have

$$
\omega_{m_{F A}}=0.4 \sqrt{\frac{g}{H_{s}}}
$$

which establishes the energy equilibrium characteristic of a fully arisen sea. The two requirements for a fully developed sea are

- sufficient distance over which the wind is blowing over the water, or fetch

- sufficient length of time the wind has been blowing, or duration.

If the fetch or duration are insufficient, the modal frequency will be greater than that given by Equation 2.12; if the winds are subsiding, the modal frequency will be less than that given by Equation 2.12. This is best understood by considering the mechanism by which waves are generated. The wind causes ripples, or capillary waves, on the sea surface, which is a short wavelength, high frequency phenomenon. Thus as a storm is building, wave energy input occurs at high frequency, and the energy gradually is transferred through wave-wave interactions to lower frequencies. Likewise, as a storm is subsiding, the high frequency waves attenuate most 
rapidly due to viscous effects, and the result is a skewing of the spectral energy toward lower frequencies. By introducing a term we will call the development factor, Dev, we can quantify the degree of sea arousal as given in Equation 2.13. [9]

$$
\omega_{m}=D e v \cdot \omega_{m_{F A}}
$$

Using this convention, values of Dev $<1.0$ correspond to a decaying sea, and values of $D e v>1.0$ correspond to a building sea. Figures 2-3 and 2-4 show representative plots of $S(\omega)$ as significant wave height and development, respectively, are varied. Note that in Figure 2-4, that while changing the development shifts the location of peak spectral energy density, it keeps the area under the curve, and thus the total energy, constant.

\subsubsection{Ochi Six Parameter Spectrum}

The Ochi six parameter spectrum is a generalization of the Bretschneider two parameter spectrum in two respects:

- two spectral peaks are present, each with their own modal frequency and significant wave height, and their contributions add together

- a shape factor, $\lambda$, can be applied to each spectral peak to skew the curve right or left, independent of the significant wave height or modal frequency. [9]

In the form given in Equation 2.8, each parameter is an independent variable. However, by analyzing over 800 wave spectra from the North Atlantic, Ochi obtained a relationship for the six parameters in terms of the measured significant wave height, $H_{s}$. [7] The result of this analysis is a family of spectra having a $95 \%$ confidence; however, the results given in Table 2.2 represent a single "most probable" spectrum [7]. The model implements this single most probable form for obtaining the six parameters of the Ochi spectrum. A sample plot of the Ochi most probable spectrum, showing its characteristic bimodal shape, is shown in Figure 2-5. 


\begin{tabular}{ll}
\hline Parameter & Value \\
\hline \hline$H_{s 1}$ & $0.84 H_{s}$ \\
$H_{s 2}$ & $0.54 H_{s}$ \\
$\omega_{m 1}$ & $0.70 e^{-0.046 H_{s}}$ \\
$\omega_{m 2}$ & $1.15 e^{-0.039 H_{s}}$ \\
$\lambda_{1}$ & 3.00 \\
$\lambda_{2}$ & $1.54 e^{-0.062 H_{s}}$ \\
\hline
\end{tabular}

Table 2.2: Ochi Six Parameter Spectrum: Most Probable Values

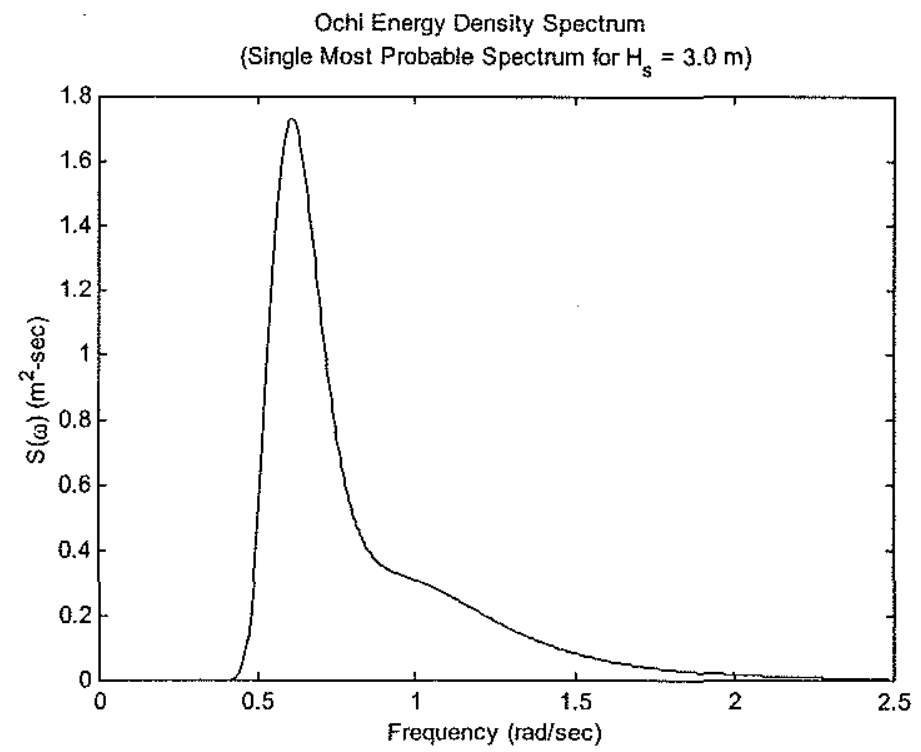

Figure 2-5: Ochi Six Parameter Single Most Probable Spectrum 


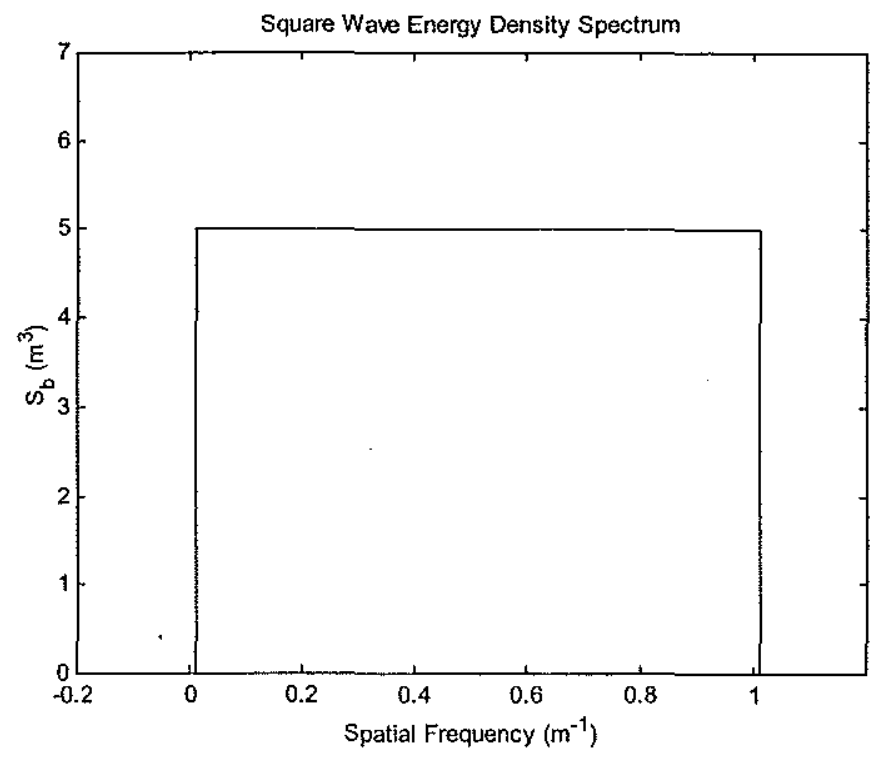

Figure 2-6: Square Wave Spectrum

\subsubsection{Square Wave Spectrum}

The square wave spectrum was added to the model to provide for verification of the resultant multidirectional wave field spectral analysis. By providing a more easily recognizable input spectrum, the goal was to generate a more easily recognizable output spectrum. A plot of the square wave spectrum is shown in Figure 2-6.

\subsubsection{Single Sine Wave Spectrum}

The single sine wave spectrum was added to the model to provide for an efficient recognizable input spectrum which could be used for troubleshooting the frequency related components of the multidirectional wave field. By providing a more easily recognizable input spectrum, the goal was to generate a more easily recognizable output spectrum. A plot of the single sine wave spectrum is shown in Figure 2-7. 


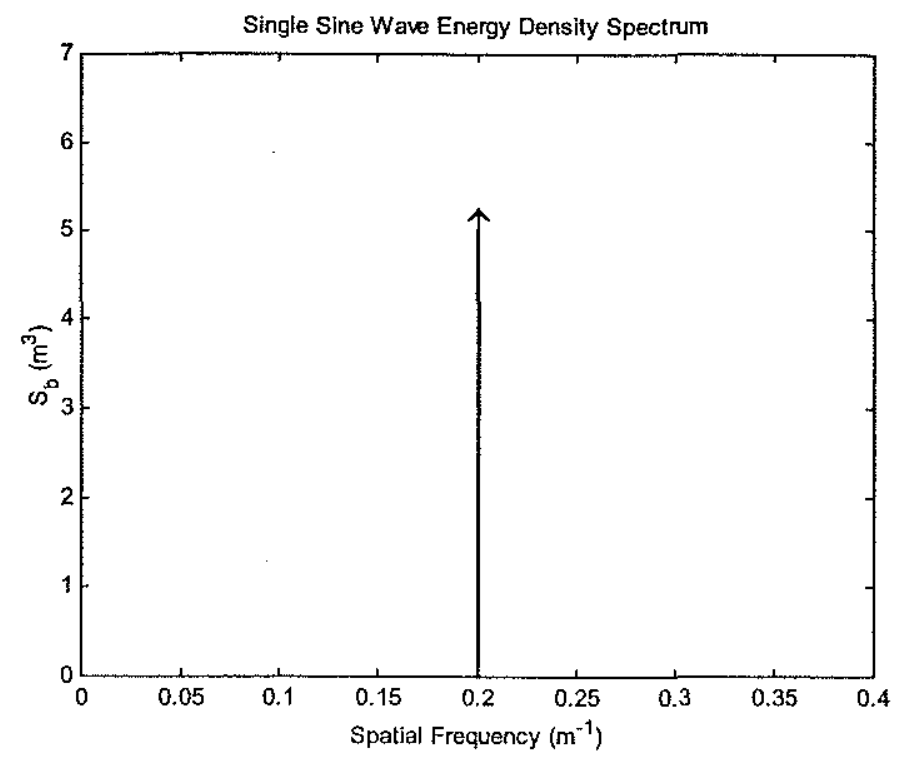

Figure 2-7: Single Sine Wave Spectrum

\subsection{Unidirectional Modeling Methods}

As mentioned earlier, the wave field in the previous model consisted of a unidirectional random seaway with the choice of only two tow directions. The wave field in the current model makes use of the unidirectional method to construct the multidirectional wave field, so it is necessary to completely understand the unidirectional method developed by Ulrich before moving on to the development of the multidirectional method. The discussion and derivation of the unidirectional method that follows comes from Ulrich. [9]

The sea domain variables of interest for the unidirectional wave field are

- sea elevation, $h$

- fluid horizontal velocity, $u$

- fluid vertical velocity, $w$.

Two methods were employed in arriving at the sea domain properties of the unidirectional wave field, each with certain advantages and disadvantages. The first method will be called the Sine Wave Superposition (SWS) method, and the second method will be called the Fast 
Fourier Transform (FFT) method. The source code for both of these computer programs can be found in reference [10].

\subsubsection{Sine Wave Superposition (SWS) Method}

As previously mentioned in Equation 2.1, the sea elevation can be obtained by a superposition of sine waves having a random phase and an amplitude prescribed by Equation 2.2. In like manner, the fluid velocities can be calculated by the superposition method. The formulas for $h, u$ and $w$ in an earth-fixed reference frame are provided in Equations. $2.14-2.16$.

$$
\begin{aligned}
& h\left(x^{\prime}, t\right)=\sum_{i=1}^{N} a_{i}(\omega) \cos \left(k_{i}(\omega) x^{\prime}-\omega_{i} t+\phi_{i}\right) \\
& u\left(x^{\prime}, t\right)=\sum_{i=1}^{N} a_{i}(\omega) \omega \cos \left(k_{i}(\omega) x^{\prime}-\omega_{i} t+\phi_{i}\right) \\
& w\left(x^{\prime}, t\right)=\sum_{i=1}^{N} a_{i}(\omega) \omega \sin \left(k_{i}(\omega) x^{\prime}-\omega_{i} t+\phi_{i}\right)
\end{aligned}
$$

By invoking a coordinate transformation and the dispersion relation from Table 2.1, one arrives at the forms appropriate to the coordinate system moving with the antenna. These are presented in Equations $2.17-2.19$.

$$
\begin{aligned}
& h(x, t)=\sum_{i=1}^{N} a_{i}(\omega) \cos \left[k_{i}(\omega) x-\left(\omega_{i}-\frac{\omega_{i}^{2} U}{g}\right) t+\phi_{i}\right] \\
& u(x, t)=\sum_{i=1}^{N} a_{i}(\omega) \omega \cos \left[k_{i}(\omega) x-\left(\omega_{i}-\frac{\omega_{i}^{2} U}{g}\right) t+\phi_{i}\right] \\
& w(x, t)=\sum_{i=1}^{N} a_{i}(\omega) \omega \sin \left[k_{i}(\omega) x-\left(\omega_{i}-\frac{\omega_{i}^{2} U}{g}\right) t+\phi_{i}\right]
\end{aligned}
$$

The spectral energy density function $S(\omega)$ used in this formulation was a single sided spectrum. The advantages of the SWS method are

- simplicity in coding fluid property subroutines

- good resolution for $S(\omega)$ at lower frequencies, where most of the wave energy resides 


\begin{tabular}{ll}
\hline $\begin{array}{l}\text { Time-Frequency } \\
\text { Domain }\end{array}$ & $\begin{array}{l}\text { Space-Spatial Fre- } \\
\text { quency Domain }\end{array}$ \\
\hline \hline$f=1 / T$ & $b=1 / L$ \\
$\omega=2 \pi f$ & $k=2 \pi b$ \\
$k=(2 \pi f)^{2} / g$ & $\omega=\sqrt{2 \pi g b}$ \\
\hline
\end{tabular}

Table 2.3: Comparison of Fourier Transform Domain Relationships

- frequency meshing $(\Delta \omega)$ is unrelated to the spatial meshing $(\Delta x)$, allowing both antenna element length and wave energy frequency resolution to be arbitrarily chosen.

\subsubsection{Fast Fourier Transform (FFT) Method}

This method takes advantage of the fact that Equations 2.14-2.16 are a Fourier series, and thus the functions $h, u$, and $w$ are the inverse Fourier transform of some expression. Since the calculations to determine the sea domain properties occur at a fixed point in time, the transform variables are not $\omega$ and $t$, rather they become $b$ and $x$. Table 2.3 summarizes the analogous relationships between the time-frequency domains and the space-spatial frequency domains. Because the FFT algorithm used for the model assumed the presence of both positive and negative frequency components, it was necessary to use a two sided spectrum for this method. In order to retain the same amount of energy (i.e. area under the spectral energy density curve) as the transition from one-sided to two-sided spectra is made, it is necessary that the formula for the component amplitudes be adjusted from that given in Equation 2.2. In addition, it is convenient to incorporate the random phase, $\phi_{i}$, into a complex component amplitude, $c_{i}$. With these changes in mind, the formulas providing the sea domain properties are given in Equations $2.20-2.24$.

$$
\begin{aligned}
& h(x)=\operatorname{Re}\left\{\sum_{i=1}^{N} c_{i}\left(b_{i}\right) \exp \left[i\left(2 \pi b_{i} x+\left(2 \pi b_{i} U-\sqrt{2 \pi g b_{i}}\right) t\right)\right]\right\} \\
& u(x)=\operatorname{Re}\left\{\sum_{i=1}^{N} c_{i}\left(b_{i}\right) \sqrt{2 \pi g b_{i}} \exp \left[i\left(2 \pi b_{i} x+\left(2 \pi b_{i} U-\sqrt{2 \pi g b_{i}}\right) t\right)\right]\right\} \\
& w(x)=\operatorname{Re}\left\{\sum_{i=1}^{N}-i c_{i}\left(b_{i}\right) \sqrt{2 \pi g b_{i}} \exp \left[i\left(2 \pi b_{i} x+\left(2 \pi b_{i} U-\sqrt{2 \pi g b_{i}}\right) t\right)\right]\right\}
\end{aligned}
$$




$$
\begin{aligned}
& \operatorname{Re}\left\{c_{i}\left(b_{i}\right)\right\}=\sqrt{\frac{1}{2} S\left(b_{i}\right) \Delta b} \cos \left(\phi_{i}\right) \\
& \operatorname{Im}\left\{c_{i}\left(b_{i}\right)\right\}=\sqrt{\frac{1}{2} S\left(b_{i}\right) \Delta b} \sin \left(\phi_{i}\right)
\end{aligned}
$$

Then the Fourier transform pairs become

$$
\begin{aligned}
H(b) & \Leftrightarrow h(x) \\
\sqrt{2 \pi g b_{i}} H(b) & \Leftrightarrow u(x) \\
-i \sqrt{2 \pi g b_{i}} H(b) & \Leftrightarrow w(x)
\end{aligned}
$$

where

$$
H(b)=\sum_{i=1}^{N} c_{i}(b) \exp \left[i t\left(2 \pi b_{i} U-\sqrt{2 \pi g b_{i}}\right)\right]
$$

and

$$
H(b) \Leftrightarrow h(x)
$$

is defined to mean

$$
\begin{aligned}
& H(b)=\sum_{i=1}^{N} h(x) \exp (-i 2 \pi b x) \\
& h(x)=\frac{1}{2 \pi} \sum_{i=1}^{N} H(b) \exp (i 2 \pi b x)
\end{aligned}
$$

By setting the complex component amplitudes, $c_{i}$, to obey the relation

$$
c_{i}\left(b_{i}\right)=\overline{c_{i}\left(-b_{i}\right)}
$$

that is, the positive frequency components are the complex conjugates of the negative frequency components, then the imaginary parts of Equations 2.20-2.22 are zero, and the $\operatorname{Re}\{\}$ can be 
dropped from the equations. Finally, the wave field is constructed by performing an inverse FFT on the one-dimensional complex arrays, represented by the left side of Equations 2.25 2.27, which contain the Fourier coefficients describing $h, u$, and $w$.

When converting between the spectral energy density function as a function of $\omega$ to another variable, such as $b$, the basic relations of Equations 2.33 and 2.34 are useful.

$$
\begin{aligned}
S(\omega) d \omega & =S(b) d b \\
S(b) & =S(\omega) \frac{d \omega}{d b}
\end{aligned}
$$

An important feature of the FFT method is the linkage of the spatial frequency domain meshing $(\Delta b)$, and the sea spatial meshing $(\Delta x)$. The key to the FFT method is to make the antenna spatial meshing, $\Delta s$, match the sea domain spatial meshing, $\Delta x$. This is accomplished by using the relations given in Equations $2.35-2.38$, and ensuring that the sea $\Delta x$ equals the desired antenna $\Delta s$.

$$
\begin{aligned}
\Delta b & =\frac{1}{L_{\text {sea }}}=b_{\min } \\
b_{\max } & =\frac{N_{\text {sea }} \Delta b}{2}=\frac{N_{\text {sea }}}{2 L_{\text {sea }}}=\frac{1}{2 \Delta x} \\
\omega_{\min } & =\sqrt{2 \pi g b_{\min }} \\
\omega_{\max } & =\sqrt{2 \pi g b_{\max }}
\end{aligned}
$$

The primary advantage of the FFT method is computational speed. The FFT method executes approximately 10 times faster than the SWS version. A further increase in execution speed is accomplished by not computing the FFT every time step, $\Delta t$. Rather, the sea data $(h, u, w)$ is computed at $n \Delta t$ intervals, and linearly interpolated in between. The integer $n$ is determined by the computer model using the highest sea energy frequency component, $\omega_{\max }$. This ensures that the linearly interpolated sea evolves in a smooth and continuous fashion.

Its disadvantage is that it has poorer low frequency resolution of the sea wave energy than its SWS counterpart. This is demonstrated by a typical run case in Table 2.4 where the lowest 10 frequencies defined are listed for both methods. As one can readily calculate from integrating Equation 2.7 with respect to $\omega$, for a sea severity of $2.0 \mathrm{~m}$ significant wave height, $67.7 \%$ of the 


\begin{tabular}{lll}
\hline Component & $\begin{array}{l}\text { Frequency, } \omega_{i} \\
\text { SWS Method }\end{array}$ & FFT Method \\
\hline \hline 1 & 0.347 & 0.347 \\
2 & 0.381 & 0.491 \\
3 & 0.414 & 0.601 \\
4 & 0.448 & 0.694 \\
5 & 0.482 & 0.776 \\
6 & 0.515 & 0.850 \\
7 & 0.549 & 0.918 \\
8 & 0.583 & 0.981 \\
9 & 0.617 & 1.041 \\
10 & 0.650 & 1.097 \\
\multicolumn{3}{l}{} \\
Notes: & \multicolumn{2}{l}{} \\
1. FFT case: $\omega \in[0.347,22.2]$ with 4096 frequency \\
components \\
2. SWS case: $\omega \in[0.347,24.0]$ with 700 frequency \\
components
\end{tabular}

Table 2.4: Comparison of Frequency Resolution for the SWS and FFT Methods

sea wave energy lies below a frequency of $1.097 \mathrm{rad} / \mathrm{sec}$. Thus the FFT method suffers in that it places most of the frequency components at high frequencies, where little of the wave energy resides. This undesirable result is a consequence of choosing a $b$ space meshing with constant $\Delta b$, which is necessary to compute FFT's in the $b$ and $x$ domains. A constant $\Delta b$ meshing produces a non-constant $\Delta \omega$ meshing with $\Delta \omega_{i}=\sqrt{2 \pi g b_{\min }}(\sqrt{i}-\sqrt{i-1})$.

\subsection{Multidirectional Modeling Methods}

The current model improves upon the previous model by upgrading the two-dimensional unidirectional wave field discussed in Section 2.3 to a three-dimensional multidirectional wave field with the choice of any arbitrary tow angle in the wave field.

The sea domain variables of interest for the multidirectional wave field are

- sea elevation, $h$

- horizontal fluid velocity (x-direction), $u$

- horizontal fluid velocity (y-direction), $v$ 
- vertical fluid velocity, $w$.

Two methods were considered in arriving at the sea domain properties of the multidirectional wave field, each with certain advantages and disadvantages. The first method will be called the Complete Wave Field (CWF) method, and the second will be called the Partial Wave Field (PWF) method.

\subsubsection{Complete Wave Field (CWF) Method}

The Complete Wave Field (CWF) method involves the construction of a three-dimensional wave field using a spectral energy model that is completely described in both the $\mathrm{x}$ and $\mathrm{y}$ directions in the spatial frequency domain. This is accomplished in a manner similar to that discussed in Section 2.3.2, except that the equations now will be a function of both $\mathrm{x}$ and $\mathrm{y}$ and will separate the horizontal velocity into $\mathrm{x}$ and $\mathrm{y}$ components.

As previously mentioned in Equation 2.1, the sea elevation can be obtained by a superposition of sine waves having a random phase and an amplitude prescribed by Equation 2.2. In like manner, the fluid velocities can be calculated by the superposition method. The formulas for $h, u, v$, and $w$ in an earth-fixed reference frame are provided in Equations $2.39-2.42$.

$$
\begin{aligned}
& h\left(x^{\prime}, y^{\prime}, t\right)= \sum_{j=1}^{N} \sum_{k=1}^{N} a_{j k}\left(\omega_{x_{j}}, \omega_{y_{k}}\right) \cos \left(k_{x_{j}}\left(\omega_{x_{j}}\right) x^{\prime}+k_{y_{k}}\left(\omega_{y_{k}}\right) y^{\prime}-\omega_{j k} t+\phi_{j k}\right) \\
& u\left(x^{\prime}, y^{\prime}, t\right)= \sum_{j=1}^{N} \sum_{k=1}^{N} a_{j k}\left(\omega_{x_{j}}, \omega_{y_{k}}\right) \omega_{j k} \cos \left(\theta_{j k}\right) \\
& \times \cos \left(k_{x_{j}}\left(\omega_{x_{j}}\right) x^{\prime}+k_{y_{k}}\left(\omega_{y_{k}}\right) y^{\prime}-\omega_{j k} t+\phi_{j k}\right) \\
& v\left(x^{\prime}, y^{\prime}, t\right)= \\
& \sum_{j=1}^{N} \sum_{k=1}^{N} a_{j k}\left(\omega_{x_{j}}, \omega_{y_{k}}\right) \omega_{j k} \sin \left(\theta_{j k}\right) \\
& \times \cos \left(k_{x_{j}}\left(\omega_{x_{j}}\right) x^{\prime}+k_{y_{k}}\left(\omega_{y_{k}}\right) y^{\prime}-\omega_{j k} t+\phi_{j k}\right)
\end{aligned}
$$




$$
w\left(x^{\prime}, y^{\prime}, t\right)=\sum_{j=1}^{N} \sum_{k=1}^{N} a_{j k}\left(\omega_{x_{j}}, \omega_{y_{k}}\right) \omega_{j k} \sin \left(k_{x_{j}}\left(\omega_{x_{j}}\right) x^{\prime}+k_{y_{k}}\left(\omega_{y_{k}}\right) y^{\prime}-\omega_{j k} t+\phi_{j k}\right)
$$

where

$$
\begin{gathered}
\theta_{j k}=\tan ^{-1}\left(\frac{k_{y_{k}}}{k_{x_{j}}}\right) \\
\omega_{j k}=\sqrt{\omega_{x_{j}}^{2}+\omega_{y_{k}}^{2}}
\end{gathered}
$$

By invoking a coordinate transformation and the dispersion relation from Table 2.1, one arrives at the forms appropriate to the coordinate system moving with the antenna. These are presented in Equations 2.45 - 2.48.

$$
\begin{aligned}
h(x, y, t)= & \sum_{j=1}^{N} \sum_{k=1}^{N} a_{j k}\left(\omega_{x_{j}}, \omega_{y_{k}}\right) \\
& \times \cos \left[k_{x_{j}}\left(\omega_{x_{j}}\right) x+k_{y_{k}}\left(\omega_{y_{k}}\right) y-\left(\omega_{j k}-\frac{\omega_{j k}^{2} U \cos \left(\theta_{j k}\right)}{g}\right) t+\phi_{j k}\right] \\
u(x, y, t)= & \sum_{j=1}^{N} \sum_{k=1}^{N} a_{j k}\left(\omega_{x_{j}}, \omega_{y_{k}}\right) \omega_{j k} \cos \left(\theta_{j k}\right) \\
& \times \cos \left[k_{x_{j}}\left(\omega_{x_{j}}\right) x+k_{y_{k}}\left(\omega_{y_{k}}\right) y-\left(\omega_{j k}-\frac{\omega_{j k}^{2} U \cos \left(\theta_{j k}\right)}{g}\right) t+\phi_{j k}\right] \\
v(x, y, t)= & \sum_{j=1}^{N} \sum_{k=1}^{N} a_{j k}\left(\omega_{x_{j}}, \omega_{y_{k}}\right) \omega_{j k} \sin \left(\theta_{j k}\right) \\
& \times \cos \left[k_{x_{j}}\left(\omega_{x_{j}}\right) x+k_{y_{k}}\left(\omega_{y_{k}}\right) y-\left(\omega_{j k}-\frac{\omega_{j k}^{2} U \cos \left(\theta_{j k}\right)}{g}\right) t+\phi_{j k}\right]
\end{aligned}
$$




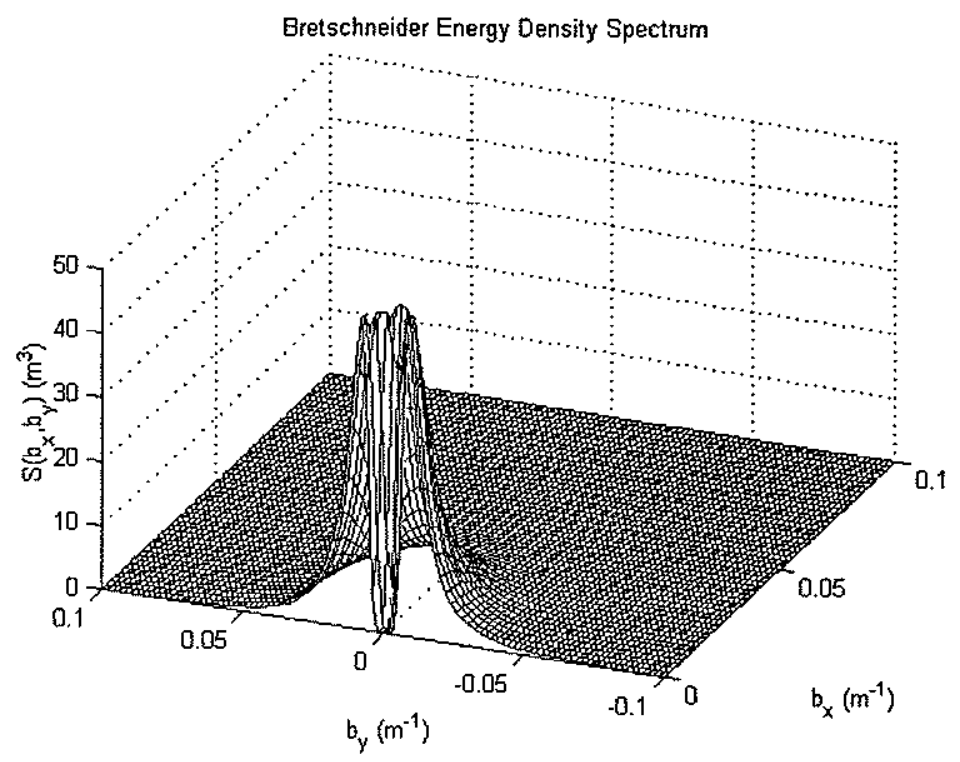

Figure 2-8: Complete Wave Field 3-D Energy Spectrum (quadrants 1 and 4 shown)

$$
\begin{aligned}
w(x, y, t)= & \sum_{j=1}^{N} \sum_{k=1}^{N} a_{j k}\left(\omega_{x_{j}}, \omega_{y_{k}}\right) \omega_{j k} \\
& \quad \times \sin \left[k_{x_{j}}\left(\omega_{x_{j}}\right) x+k_{y_{k}}\left(\omega_{y_{k}}\right) y-\left(\omega_{j k}-\frac{\omega_{j k}^{2} U \cos \left(\theta_{j k}\right)}{g}\right) t+\phi_{j k}\right]
\end{aligned}
$$

We again take advantage of the fact that Equations $2.45-2.48$ are a Fourier series, and thus the functions $h, u, v$, and $w$ are the inverse Fourier transform of some expression. Since the calculations to determine the sea domain properties occur at a fixed point in time, the transform variables are not $\omega$ and $t$, rather they become $b$ and $x$. Because the two-dimensional FFT algorithm used for the model assumed the presence of frequency components in all four quadrants surrounding the origin in the spatial frequency domain, it was necessary to construct an energy density spectrum in three dimensions. An example of a three-dimensional energy density spectrum, in spatial frequency coordinates, which is used by the sea domain properties equations is shown in Figure 2-8. In order to retain the same amount of energy (i.e. area under the spectral energy density curve), it is necessary that the formula for the component amplitudes be adjusted from that given in Equation 2.2. In addition, it is convenient to incorporate the 
random phase, $\phi_{j k}$, into a complex component amplitude, $c_{j k}$. With these changes in mind, the formulas providing the sea domain properties are given in Equations $2.49-2.54$.

$$
\begin{aligned}
& h(x, y)=\sum_{j=1}^{N} \sum_{k=1}^{N} \operatorname{Re}\left\{c_{j k}\left(b_{x_{j}}, b_{y_{k}}\right)\right. \\
& \left.\times \exp \left[i\left(2 \pi b_{x_{j}} x+2 \pi b_{y_{k}} y+\left(2 \pi b_{j k} U \cos \left(\theta_{j k}\right)-\sqrt{2 \pi g b_{j k}}\right) t\right)\right]\right\} \\
& u(x, y)=\sum_{j=1}^{N} \sum_{k=1}^{N} \operatorname{Re}\left\{c_{j k}\left(b_{x_{j}}, b_{y_{k}}\right) \sqrt{2 \pi g b_{j k}} \cos \left(\theta_{j k}\right)\right. \\
& \left.\times \exp \left[i\left(2 \pi b_{x_{j}} x+2 \pi b_{y_{k}} y+\left(2 \pi b_{j k} U \cos \left(\theta_{j k}\right)-\sqrt{2 \pi g b_{j k}}\right) t\right)\right]\right\} \\
& v(x, y)=\sum_{j=1}^{N} \sum_{k=1}^{N} \operatorname{Re}\left\{c_{j k}\left(b_{x_{j}}, b_{y_{k}}\right) \sqrt{2 \pi g b_{j k}} \sin \left(\theta_{j k}\right)\right. \\
& \left.\times \exp \left[i\left(2 \pi b_{x_{j}} x+2 \pi b_{y_{k}} y+\left(2 \pi b_{j k} U \cos \left(\theta_{j k}\right)-\sqrt{2 \pi g b_{j k}}\right) t\right)\right]\right\} \\
& w(x, y)=\sum_{j=1}^{N} \sum_{k=1}^{N} \operatorname{Re}\left\{-i c_{j k}\left(b_{x_{j}}, b_{y_{k}}\right) \sqrt{2 \pi g b_{j k}}\right. \\
& \left.\times \exp \left[i\left(2 \pi b_{x_{j}} x+2 \pi b_{y_{k}} y+\left(2 \pi b_{j k} U \cos \left(\theta_{j k}\right)-\sqrt{2 \pi g b_{j k}}\right) t\right)\right]\right\} \\
& \operatorname{Re}\left\{c_{j k}\left(b_{x_{j}}, b_{y_{k}}\right)\right\}=\sqrt{\frac{1}{2 b_{j k}} S\left(b_{x_{j}}, b_{y_{k}}\right)} \Delta b \cos \left(\phi_{j k}\right) \\
& \operatorname{Im}\left\{c_{j k}\left(b_{x_{j}}, b_{y_{k}}\right)\right\}=\sqrt{\frac{1}{2 b_{j k}} S\left(b_{x_{j}}, b_{y_{k}}\right)} \Delta b \sin \left(\phi_{j k}\right)
\end{aligned}
$$

where

$$
b_{j k}=\sqrt{b_{x_{j}}^{2}+b_{y_{k}}^{2}}
$$


Then the Fourier transform pairs become

$$
\begin{aligned}
H\left(b_{x}, b_{y}\right) & \Leftrightarrow h(x, y) \\
\sqrt{2 \pi g b_{j k}} \cos \left(\theta_{j k}\right) H\left(b_{x}, b_{y}\right) & \Leftrightarrow u(x, y) \\
\sqrt{2 \pi g b_{j k}} \sin \left(\theta_{j k}\right) H\left(b_{x}, b_{y}\right) & \Leftrightarrow v(x, y) \\
-i \sqrt{2 \pi g b_{j k}} H\left(b_{x}, b_{y}\right) & \Leftrightarrow w(x, y)
\end{aligned}
$$

where

$$
H\left(b_{x}, b_{y}\right)=\sum_{j=1}^{N} \sum_{k=1}^{N} c_{j k}\left(b_{x_{j}}, b_{y_{k}}\right) \exp \left[i t\left(2 \pi b_{j k} U \cos \left(\theta_{j k}\right)-\sqrt{2 \pi g b_{j k}}\right)\right]
$$

and

$$
H\left(b_{x}, b_{y}\right) \Leftrightarrow h(x, y)
$$

is defined to mean

$$
\begin{aligned}
H\left(b_{x}, b_{y}\right) & =\sum_{j=1}^{N} \sum_{k=1}^{N} h\left(x_{j}, y_{k}\right) \exp \left(-i 2 \pi b_{x_{j}} x-i 2 \pi b_{y_{k}} y\right) \\
h(x, y) & =\frac{1}{2 \pi} \sum_{j=1}^{N} \sum_{k=1}^{N} H\left(b_{x_{j}}, b_{y_{k}}\right) \exp \left(i 2 \pi b_{x_{j}} x+i 2 \pi b_{y_{k}} y\right)
\end{aligned}
$$

By setting the complex component amplitudes, $c_{j k}$, to obey the relations

$$
\begin{aligned}
c_{j k}\left(-b_{x_{j}},-b_{y_{k}}\right) & =\overline{c_{i}\left(b_{x_{j}}, b_{y_{k}}\right)} \\
c_{j k}\left(-b_{x_{j}}, b_{y_{k}}\right) & =\overline{c_{i}\left(b_{x_{j}},-b_{y_{k}}\right)}
\end{aligned}
$$

that is, the frequency components of quadrants 2 and 3 are the complex conjugates of the frequency components of quadrants 4 and 1, respectively, then the imaginary parts of Equations $2.49-2.52$ are zero, and the $\operatorname{Re}\{\}$ can be dropped from the equations.

Finally, the wave field is constructed by performing an inverse FFT on the two-dimensional complex arrays, represented by the left side of Equations $2.56-2.59$, which contain the Fourier 


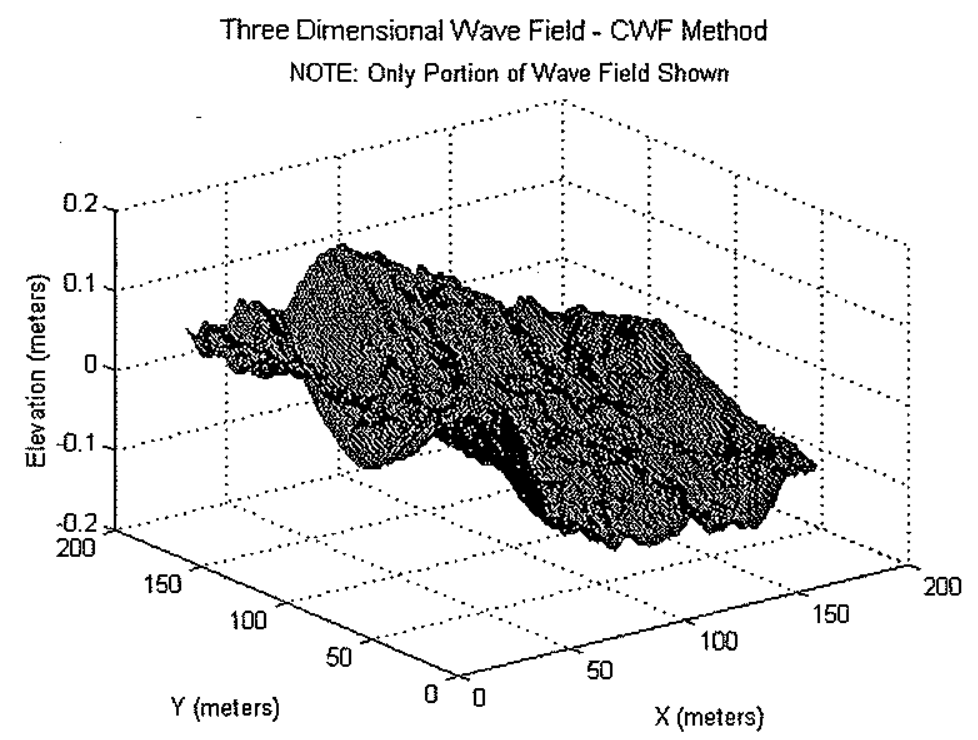

Figure 2-9: Wave Field Generated by CWF Method

coefficients describing $h, u, v$, and $w$. A example of the wave field generated by the CWF method is shown in Figure 2-9. This computational step is not trivial and is the reason that this method was abandoned early in the project. The memory requirements and computation times necessary to complete the inverse FFT of these large arrays was not practical for desktop computer modeling.

Wave field directionality has not been discussed up to this point. That topic has been reserved for Section 2.4.2, the Partial Wave Field method, which is the method that is implemented in the model.

\subsubsection{Partial Wave Field (PWF) Method}

The Partial Wave Field (PWF) method involves the construction of a three-dimensional wave field using a spectral energy model that is only partially described in both the $\mathrm{x}$ and $\mathrm{y}$ directions in the spatial frequency domain. In comparison to the CWF method, this partial description significantly reduces the amount of computation needed to build the modeled wave field. It reduces computation time by forming the multidirectional wave field from the proper combination of several unidirectional waves as shown in Figure 2-10. In addition, the PWF method 


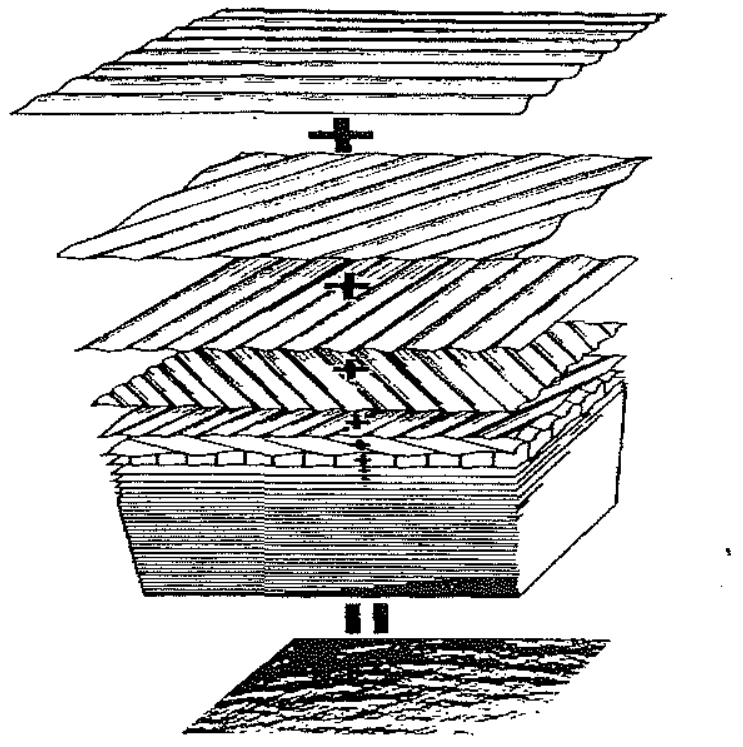

Figure 2-10: PWF Unidirectional Wave Summation

takes advantage of the fact that the only wave field information necessary to compute exposure statistics on the antenna is the information from the wave field that is directly in contact with the antenna.

The first step of the PWF method is to select, from the available choices, the wave energy density spectrum to be modeled. The energy density spectrum dictates the behavior and magnitude of the modeled seas and its selection can significantly affect the exposure statistics. Guidance on proper selection of the energy density spectrum can be found in Section A.2.1 and in numerous texts and papers. [4][5][7][8]

The next step of the PWF method is to construct the three dimensional energy density spectrum with $N N$ discrete unidirectional spectra that are each propagating in a different direction. The model allows for the user to select any odd number of discrete unidirectional waves. The requirement for an odd number is driven by the assumption that one direction lies on the principal axis and the remaining directions are split evenly on either side of the principal axis. A larger number of directions will result in a more realistic three dimensional sea spectrum but at the expense of computation time. Referring again to Figure 2-10, one can see that as the number of unidirectional waves increases, the modeled seas more closely approach a realistic 


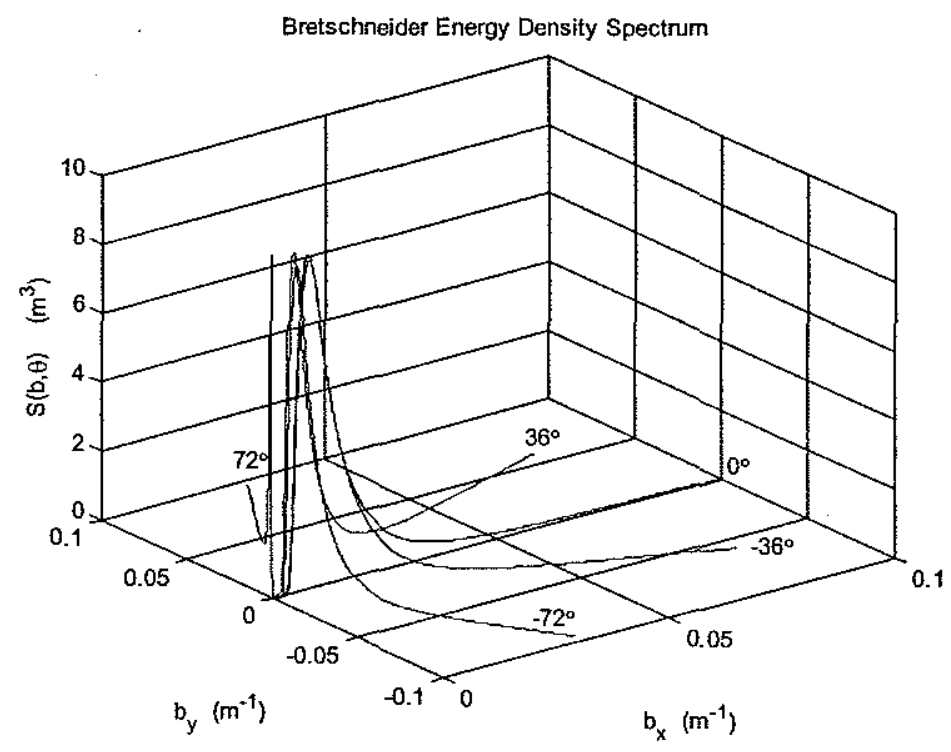

Figure 2-11: Partial Wave Field 3-D Energy Spectrum - No Directional Scaling (quadrants 1 and 4 shown)

three dimensional spectrum. Likewise, as the number of unidirectional waves decreases, the modeled seas move further away from a realistic three dimensional spectrum. The model constructs the three dimensional energy density spectrum by assigning a direction to each of the discrete unidirectional spectra, as shown in Figure 2-11. The example shown in Figure 2-11 is for five propagation directions. For a given number of propagation directions, five in this example, the model assumes equal angles between each direction and then solves for the correct angle to assign to each direction. It should be noted that, for purposes of illustration, the magnitude of each of the unidirectional spectra is equal and that Figure 2-11 is similar to Figure 2-8 except that Figure 2-11 is only partially modeling the three dimensional energy density spectrum.

It has already been pointed out the magnitude of each of the unidirectional spectra in Figure 2-11 is equal. This implies that the wave field has no directional spreading characteristic; that is, energy is being propagated equally in all directions. In reality, wind-generated waves tend to propagate in the same general direction as the wind. Typically, the energy is spread over several directions, but the majority is in the direction of the wind. This spreading of energy 
can be characterized by what is known as a directional spreading function. The directional spreading function assigns a fraction of the total wave energy to each particular direction, with that fraction being determined by the shape of the spreading model selected. To generate a directional spectrum, one simply multiplies the energy density spectrum by the directional spreading function

$$
S(\omega, \theta)=S(\omega) D(\theta, \omega)
$$

where $D(\theta, \omega)$ represents the directional spreading as a function of both direction, $\theta$, and frequency, $\omega$. Not unlike wave spectra, numerous directional spreading functions have been proposed by researchers attempting to characterize a particular sea wave environment. [4][5][6][7][8] Although the spreading functions implemented in the model are simple models that are a function of direction only, spreading models exist that represent the spreading as a function of both direction and frequency. In these frequency dependent spreading functions, the low frequency components are assigned a very narrow spreading while the high frequency components are assigned a wider spreading. [5] The spreading functions used in the model are the cosine square formula and the cosine fourth formula, given in Equations 2.67 and 2.68, respectively.

$$
\begin{array}{ll}
D(\theta)=\frac{2}{\pi} \cos ^{2}(\theta), & -\frac{\pi}{2} \leq \theta \leq \frac{\pi}{2} \\
D(\theta)=\frac{8}{3 \pi} \cos ^{4}(\theta), & -\frac{\pi}{2} \leq \theta \leq \frac{\pi}{2}
\end{array}
$$

A plot of the cosine square formula, shown in Figure 2-12, shows the behavior of the directional spreading function as the propagation direction changes from 0 degrees, the principal axis of propagation, to \pm 90 degrees. As shown in Figure 2-12, the largest fraction of energy is along the principal axis while at \pm 90 degrees the fraction tapers to zero. To account for the total energy in the spreading spectrum, the actual fraction assigned to each unidirectional spectrum is the integral of the directional spreading function centered on each direction with integration limits being equal distances on either side of the propagation direction. Again, using the five propagation direction example, the propagation directions and their respective integration limits are shown in Figure 2-13.

Applying the cosine square spreading function to the three dimensional energy density spec- 


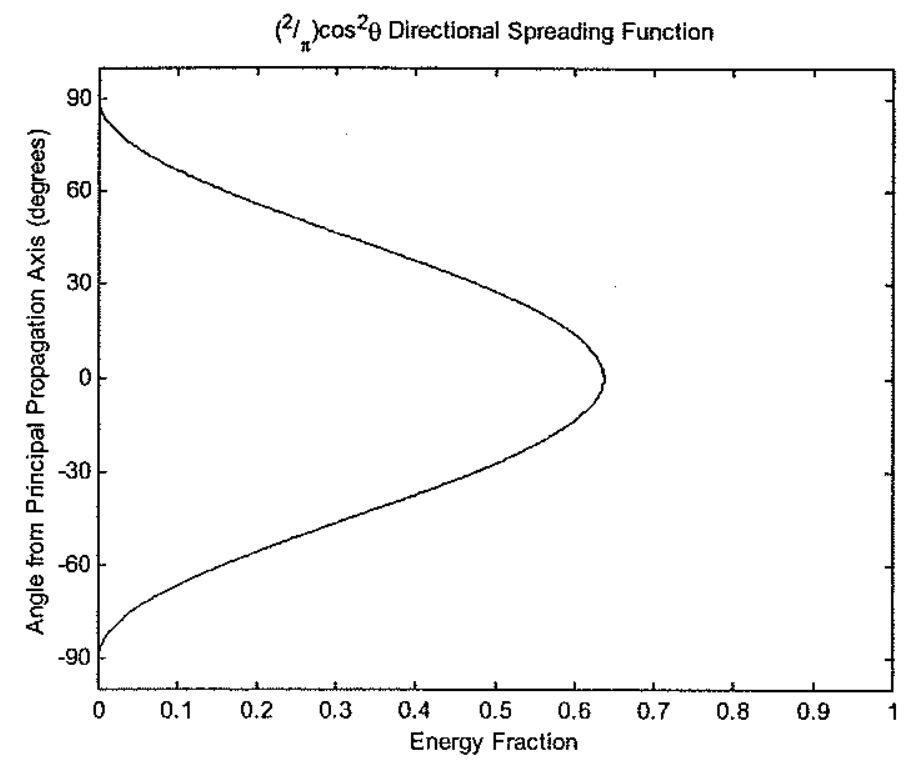

Figure 2-12: Cosine Square Directional Spreading Function

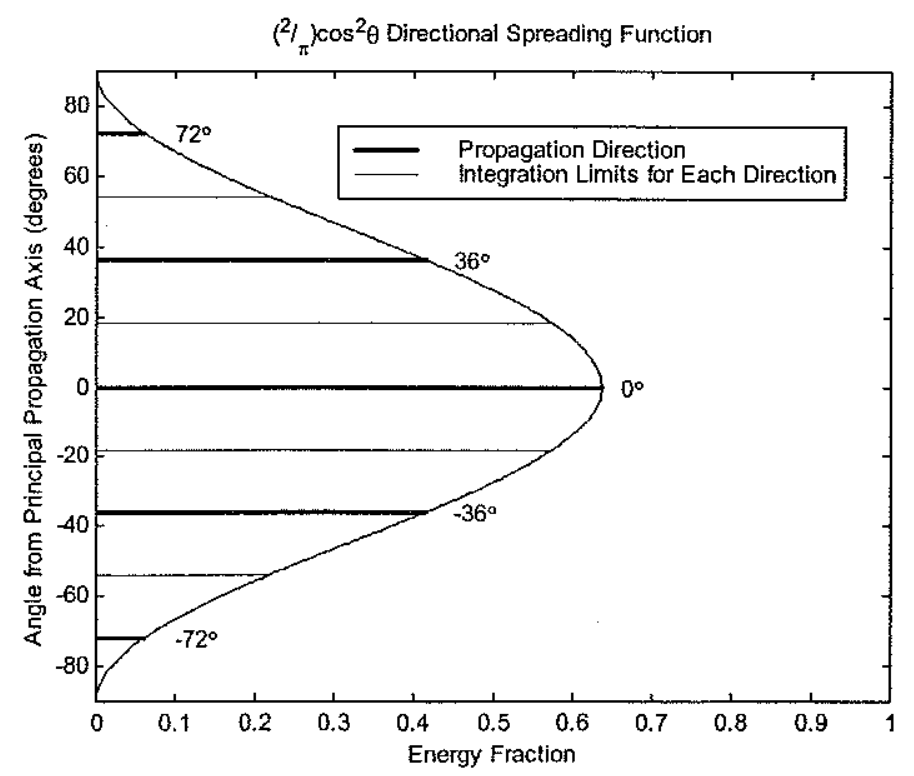

Figure 2-13: Cosine Square Directional Spreading Function for the Five Directions Example Problem 


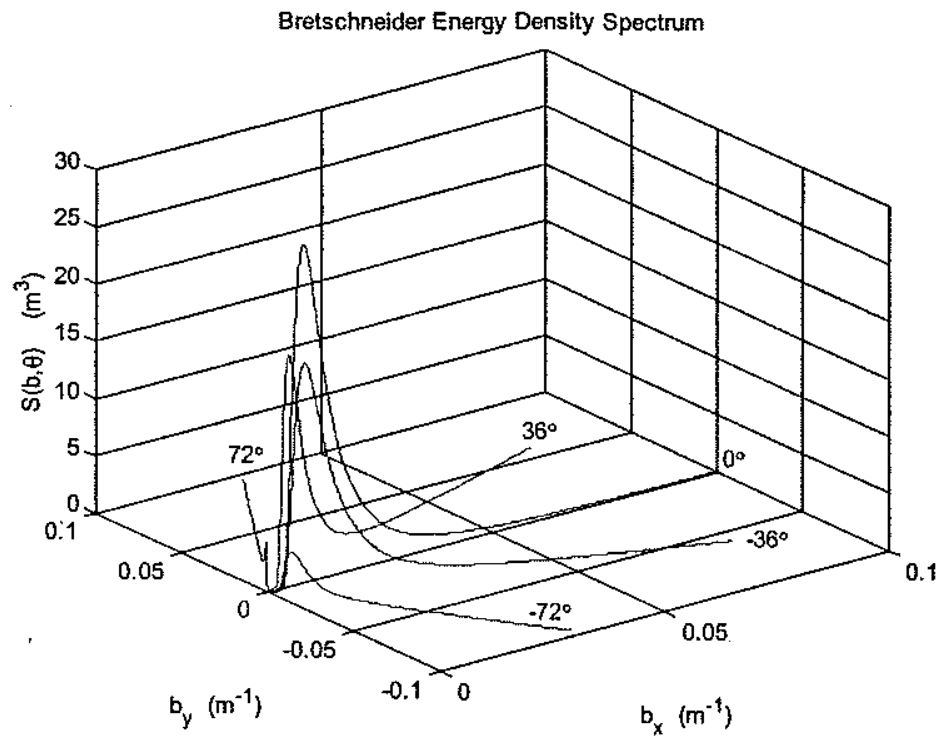

Figure 2-14: Partial Wave Field 3-D Energy Spectrum - With Directional Scaling (quadrants 1 and 4 shown)

trum in Figure 2-11, you obtain a three dimensional energy density spectrum with directional characteristics, shown in Figure 2-14. Now, comparing Figure 2-14 with Figure 2-11, you can see that the magnitudes of the unidirectional spectra in Figure 2-14 are not equal, but are scaled according to the directional spreading function. In addition, as prescribed by the spreading function, the principal axis of wave propagation, 0 degrees, contains the largest fraction of energy.

The next step in the PWF method is to apply the FFT method, described in Section 2.3.2, to each of the scaled unidirectional wave spectra to solve for the spatial sea domain properties of each unidirectional wave. Now the individual waves must be properly summed to determine the three dimensional wave properties along the antenna. The sea domain properties of the PWF method are somewhat different than those found by the CWF method. In the CWF method, the goal was to solve for $h, u, v$, and $w$ in the entire wave field. With the PWF method, the only information needed is the information along the antenna. To simplify the numerical modeling, the velocities of interest were found relative to the anitenna. So rather than solving for the $\mathrm{x}$ and $\mathrm{y}$ velocities in the entire field, the model solves for the velocities 
that act longitudinally and transversely on the antenna, as well as the vertical velocity. The summation is accomplished by Equations $2.69-2.72$.

$$
\begin{gathered}
h(x)=\sum_{i=1}^{N N} h_{i}(\widetilde{x}) \\
u_{\text {longitudinal }}(x)=\sum_{i=1}^{N N} u_{i}(\widetilde{x}) \cos (\psi) \\
u_{\text {transverse }}(x)=\sum_{i=1}^{N N} u_{i}(\widetilde{x}) \sin (\psi) \\
w(x)=\sum_{i=1}^{N N} w_{i}(\widetilde{x})
\end{gathered}
$$

where

$$
\widetilde{x}=\left\{\frac{N_{\text {sea }}}{2}-0.5\right\} \Delta s-\left\{\frac{N}{2}-\left[\frac{x}{L}(N-1)+1\right]+0.5\right\} \Delta s \cos (\psi)
$$

and

$N N$ is the number of propagation directions being summed

$h_{i}(\widetilde{x})$ is the wave elevation of the individual unidirectional wave, at the location $\widetilde{x}$, in the unidirectional wave field that is acting on the antenna location $x$, as shown in Figure 2-15

$u_{i}(\widetilde{x})$ is the horizontal velocity of the individual unidirectional wave, at the location $\widetilde{x}$, in the unidirectional wave field that is acting on the antenna location $x$, as shown in Figure 2-15

$\psi$ is the angle between the individual unidirectional wave and the antenna, as shown in Figure 2-15

$w_{i}(\widetilde{x})$ is the vertical velocity of the individual unidirectional wave, at the location $\widetilde{x}$, in the unidirectional wave field that is acting on the antenna location $x$, as shown in Figure 2-15

$N_{\text {sea }}$ is the number of spatial divisions in the unidirectional wave field

$\Delta s$ is the spatial increment on the antenna as well as the spatial frequency increment of the 


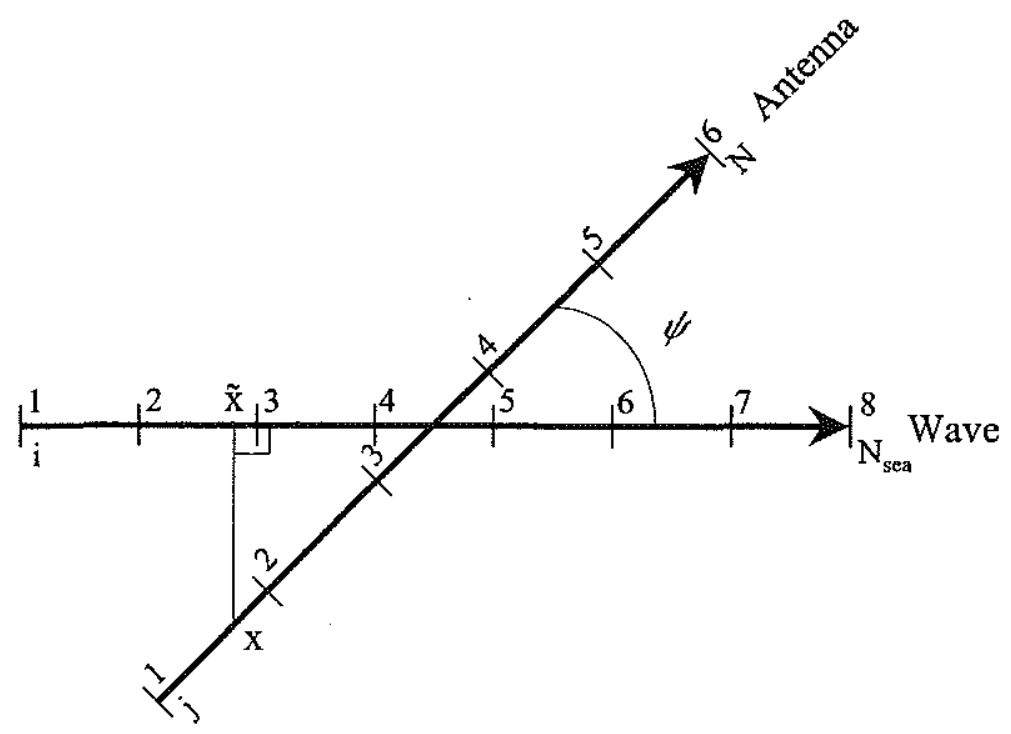

Figure 2-15: Geometry of the Wave Summation

unidirectional wave spectrum

$N$ is the number of spatial divisions on the antenna

$L$ is the modeled length of the antenna

In Equations $2.69-2.73$, it is assumed that the width of the unidirectional wave is infinite and that the surface properties along that infinite width do not vary. This assumption allows you to perform the summation shown in Figure 2-15.

The origin of equation 2.73 is not obvious, but it represents the trigonometric and geometric relation between the individual wave and the antenna. Referring to Figure 2-15, the numbers represent the array storage locations of the particular variables with the wave properties numbered $i=1$ to $N_{s e a}$ and the antenna properties numbered $j=1$ to $N$. The spacing between points, $\Delta s$, is the same for both the antenna and the wave with the length of the wave field being $L_{\text {sea }}$ and the length of the antenna being $L$. In addition, it is assumed that the antenna and the wave cross at their midpoints. The position, $\widetilde{x}$, can be found from

$$
\widetilde{x}=\left(\widetilde{x}_{\text {crossing point }}-\widetilde{x}_{\text {origin }}\right)-\left(x_{\text {crossing point }}-x\right) \cos (\psi)^{a}
$$


where

$\widetilde{x}_{\text {crossing point }}=$ (number of whole and partial mesh spaces on wave field between crossing point and origin $) \times(\Delta s)$

$$
\begin{aligned}
& =\left(\frac{N_{\text {sea }}}{2}-0.5\right) \Delta s \\
\widetilde{x}_{\text {origin }} & =0.0
\end{aligned}
$$

$x_{\text {crossing point }}=$ (number of whole and partial mesh spaces on antenna between crossing point and origin $) \times(\Delta s)$

$$
\begin{aligned}
= & \left(\frac{N}{2}-0.5\right) \Delta s \\
x= & \text { (number of whole and partial mesh spaces on antenna between } \\
& x \text { and origin) } \times(\Delta s) \\
= & (j-1) \Delta s
\end{aligned}
$$

combining equations $2.74-2.79$ you obtain

$$
\widetilde{x}=\left(\frac{N_{\text {sea }}}{2}-0.5\right) \Delta s-\left(\frac{N}{2}-j+0.5\right) \Delta s \cos (\psi)
$$

where $j$ represents the array storage location nearest to $x$, given by

$$
j=\frac{x}{L}(N-1)+1
$$

when Equation 2.81 is combined with Equation 2.80 , you obtain

$$
\widetilde{x}=\left\{\frac{N_{\text {sea }}}{2}-0.5\right\} \Delta s-\left\{\frac{N}{2}-\left[\frac{x}{L}(N-1)+1\right]+0.5\right\} \Delta s \cos (\psi)
$$

which is identical to Equation 2.73.

The PWF modeling framework consists of the following steps

1. select the wave energy density spectrum to be modeled

2. construct the three dimensional energy density spectrum with $N N$ discrete unidirectional spectra that are each propagating in a different direction 
3. select the directional spreading function

4. apply the directional spreading function to the discrete components of the three dimensional energy density spectrum

5. perform an inverse FFT on the one-dimensional complex arrays, represented by the left side of Equations $2.25-2.27$, which contain the Fourier coefficients describing $h, u$, and $w$ of each discrete unidirectional spectrum

6. properly sum the sea domain properties of each discrete unidirectional wave along the length of the antenna to generate a three dimensional wave field on the antenna

7. time advance the Fourier coefficients describing $h, u$, and $w$ using Equation 2.28

8. repeat steps 5-7 for duration of simulation.

\subsection{Verification of Model Seas}

The random multidirectional seaway was constructed by the PWF method as described in Section 2.4.2. By running the modeling code and sampling the wave field for several parameters, one can build a wave record that can be analyzed to determine if the resultant wave field correctly models the desired wave field. The modeled seas verification focused on the following four areas

- significant wave height, $H_{s}$

- encounter frequency, $\omega_{e}$

- spectral analysis of a spatial wave record

- spectral analysis of a temporal wave record.

\subsubsection{Significant Wave Height}

By sampling the wave record for sea surface elevation, one can determine the significant wave height of the sample, as defined in Section 2.2. [7] This can then be compared to the significant 


\begin{tabular}{ccccccc}
\hline & \multicolumn{3}{c}{ Spatial } & \multicolumn{3}{c}{ Temporal } \\
Sample & Mean $\left(h_{i}\right)$ & $\widehat{\sigma}$ & $\widehat{H}_{s}$ & Mean $\left(h_{i}\right)$ & $\widehat{\sigma}$ & $\widehat{H}_{s}$ \\
& $(\mathrm{~m})$ & $(\mathrm{m})$ & $(\mathrm{m})$ & $(\mathrm{m})$ & $(\mathrm{m})$ & $(\mathrm{m})$ \\
\hline 1 & -0.0546 & 0.7177 & 2.8779 & 0.0102 & 0.7784 & 3.1215 \\
2 & -0.0120 & 0.7455 & 2.9893 & 0.0032 & 0.6950 & 2.7869 \\
3 & 0.0024 & 0.8389 & 3.3638 & -0.0009 & 0.7893 & 3.1652 \\
4 & 0.0160 & 0.8890 & 3.5647 & 0.0085 & 0.7582 & 3.0405 \\
5 & 0.0287 & 0.6336 & 2.5406 & 0.0231 & 0.7017 & 2.8137 \\
6 & -0.0119 & 0.8843 & 3.5459 & -0.0045 & 0.7159 & 2.8707 \\
7 & 0.0066 & 0.7410 & 2.9713 & 0.0057 & 0.7528 & 3.0186 \\
8 & -0.0844 & 0.7636 & 3.0621 & -0.0171 & 0.7140 & 2.8632 \\
9 & -0.0362 & 0.5490 & 2.2016 & -0.0185 & 0.7412 & 2.9723 \\
10 & -0.0391 & 0.8569 & 3.4360 & 0.0092 & 0.7373 & 2.9567 \\
11 & 0.0632 & 0.7047 & 2.8258 & -0.0182 & 0.7215 & 2.8934 \\
12 & 0.0050 & 0.6758 & 2.7100 & -0.0061 & 0.7636 & 3.0619 \\
\hline Averages: & -0.0097 & 0.7500 & $\mathbf{3 . 0 0 7 5}$ & -0.0005 & 0.7391 & 2.9637 \\
\hline
\end{tabular}

Table 2.5: Simulated Significant Wave Heights for a Specified Sea Severity of $3.0 \mathrm{~m}$

wave height which was specified in the input file. The measured significant wave height can be found by Equations 2.83 and 2.84,

$$
\begin{aligned}
\widehat{\sigma}^{2} & =\frac{1}{n-1} \sum_{i=1}^{n} x_{i}^{2} \\
\widehat{H}_{s} & =4.01 \sqrt{\widehat{\sigma}^{2}}
\end{aligned}
$$

where

$x_{i}$ is the sampled wave amplitude, measured from the mean free-surface

$\widehat{\sigma}$ is the standard deviation of the sampled wave amplitudes

$\widehat{H}_{s}$ is the measured significant wave height of the wave record.

(The " - " symbol denotes that the quantity is computed from a sampled wave record, and therefore may deviate somewhat from the theoretical value.)

A sea severity of $3.0 \mathrm{~m}$ significant wave height was simulated by the PWF method. Twelve wave amplitude records were analyzed by sampling the seas spatially at $0.0625 \mathrm{~m}$ intervals and temporally at $0.021 \mathrm{sec}$ intervals. The results are summarized in Table 2.5. An excellent agreement to the specified significant wave height is achieved with an average deviation in $\widehat{H}_{s}$ of $0.25 \%$ and $1.20 \%$ for the spatial and temporal analysis, respectively. 


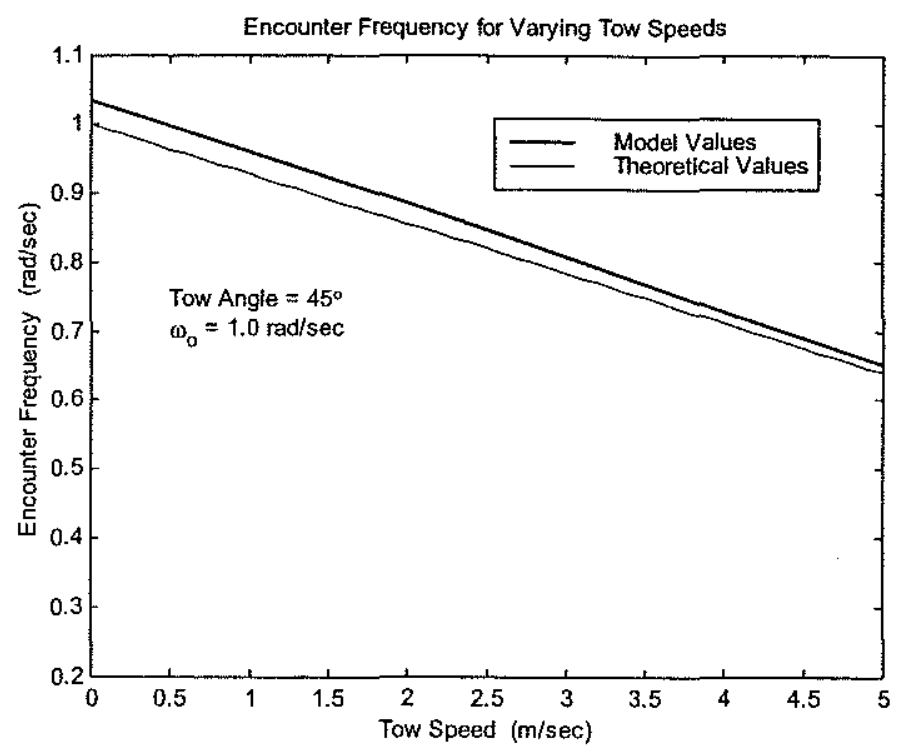

Figure 2-16: Encounter Frequency for Varying Tow Speeds

\subsubsection{Encounter Frequency}

Determination of the encounter frequency presents a formidable task for a random multidirectional seaway. To simplify the task, the analysis of encounter frequency was done using the single sine wave spectrum as the input spectrum. The method used to verify the encounter frequency invoked the superposition principle: the sea waves generated by the PWF method are ultimately a superposition of individual sine waves, thus the verification of encounter frequency was restricted to seas represented by a single sine wave. The goal of this analysis is to verify that the antenna sees the correct "encounter frequency," i.e. an effective change of the wave frequency as the antenna is towed in a seaway. The encounter frequency, $\omega_{e}$, is given by

$$
\omega_{e}=\omega_{0}-\frac{\omega_{0}^{2} U \cos (\theta)}{g}
$$

where

$\omega_{0}$ is the wave frequency in a stationary coordinate system

$U$ is the antenna tow speed

$\theta$ is the tow angle with respect to the principal axis of wave propagation 


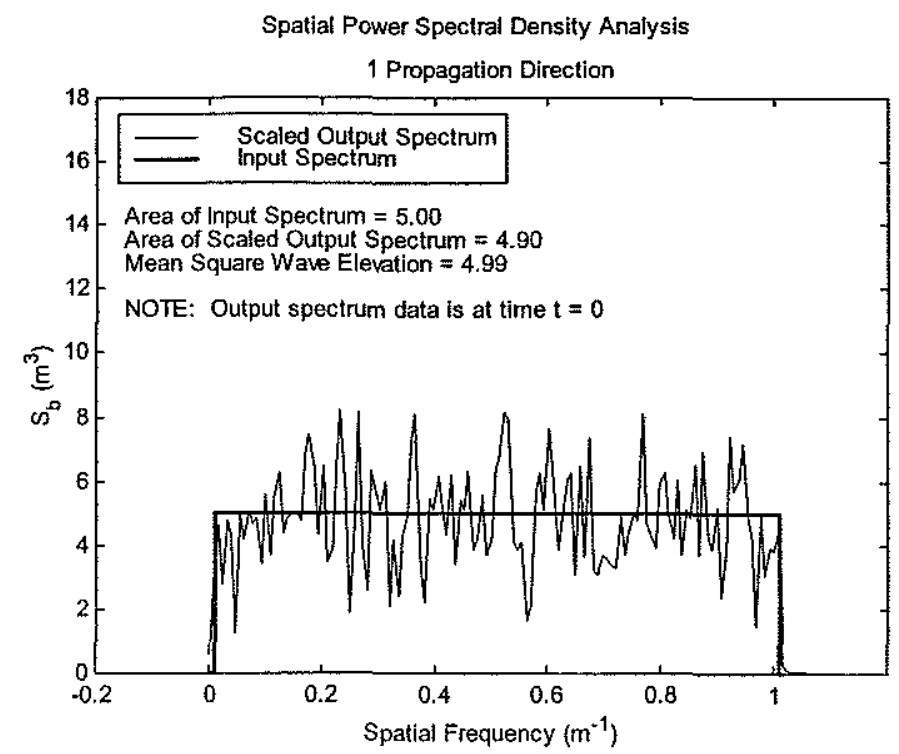

Figure 2-17: Spectral Analysis of Spatial Wave Data - One Propagation Direction

$g$ is the acceleration due to gravity.

Thus for towing into head seas $\left(\cos \left(180^{\circ}\right)\right.$ is negative), the encounter frequency goes up, and for towing with following seas $\left(\cos \left(0^{\circ}\right)\right.$ is positive), the encounter frequency goes down. The encounter frequency was measured for a single sine wave of frequency $\omega_{0}=1.0 \mathrm{rad} / \mathrm{sec}$ at tow speeds from $0.0 \mathrm{~m} / \mathrm{sec}$ to $5.0 \mathrm{~m} / \mathrm{sec}$ at a tow angle of $45^{\circ}$. The resulting plot of $\omega_{e}$ vs. $U$ is shown in Figure 2-16. The plot of $\omega_{e}$ vs. $U$ is expected to be linear with a slope of $-\frac{\omega_{0}^{2} \cos (\theta)}{g}$ (see Equation 2.85), which for $\omega_{0}=1.0 \mathrm{rad} / \mathrm{sec}$ and $\theta=45^{\circ}$ has a value of $-0.0721 \mathrm{~m}^{-1}$. The measured slope was $-0.0767 \mathrm{~m}^{-1}$, which shows the proper dependence of encounter frequency on speed has been captured by the model.

\subsubsection{Spatial Spectral Analysis}

By sampling the wave record spatially for sea surface elevation, one can determine the spatial power spectral density curve of the modeled wave field. By comparing the resultant spectral density curve to the desired spectral density curve, a verification of the model's wave field generation method can be conducted. Figure 2-17 shows the spectral analysis of spatial wave data for a wave field constructed with only one propagation direction. The resultant energy 


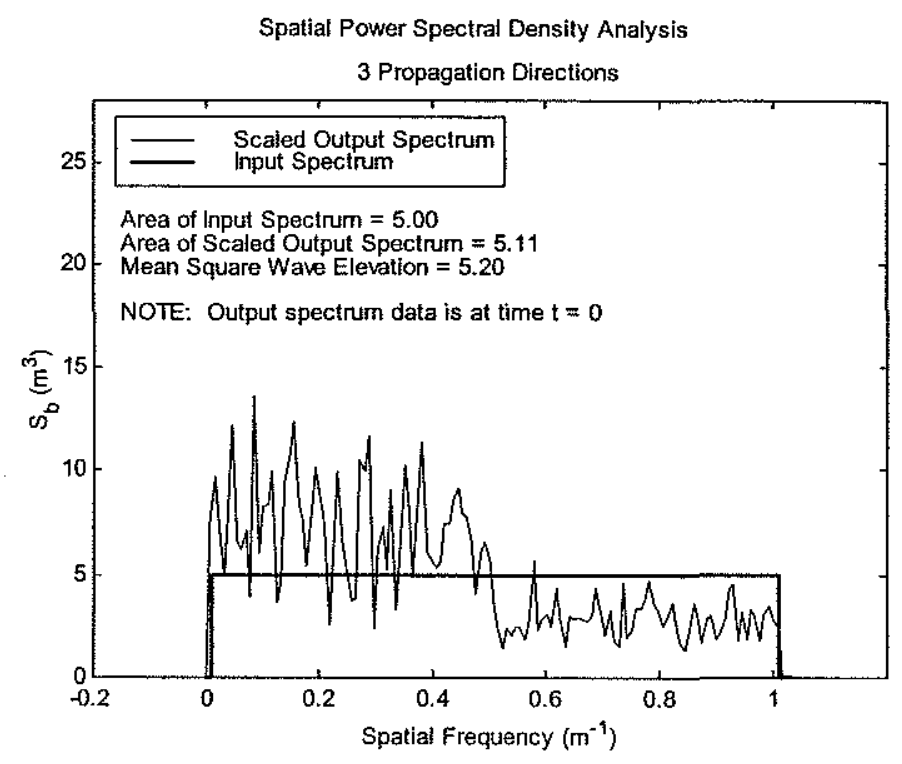

Figure 2-18: Spectral Analysis of Spatial Wave Data - Three Propagation Directions

density spectrum closely resembles the desired input spectrum and there is good agreement amongst the values representing the area under the input spectrum, the area under the output spectrum, and the mean square wave elevation of the modeled wave field.

An interesting phenomena occurs when the number of propagation directions is increased to three. As shown in Figure 2-18, there is an apparent increase in energy density at the lower spatial frequencies while the opposite occurs at the higher spatial frequencies. There is still good agreement amongst the values representing the area under the input spectrum, the area under the output spectrum, and the mean square wave elevation of the modeled wave field, but there is an obvious shift in energy to lower frequencies. This shift in energy to lower frequencies is attributed to the wave summation method listed in Equation 2.73 and shown in Figure 2-15. As the individual wave is added, it is being "stretched" in length due to the angle between the antenna and the wave itself. For the extreme case where this angle is $90^{\circ}$, only one value of wave elevation from the individual wave would be applied to the entire length of the antenna. In this case, a single point value is stretched along the entire length of the antenna. In the opposite extreme case where the angle is $0^{\circ}$, there would be a point to point match in the individual wave and the antenna with no stretching, as shown in Figure 2-17. The same 


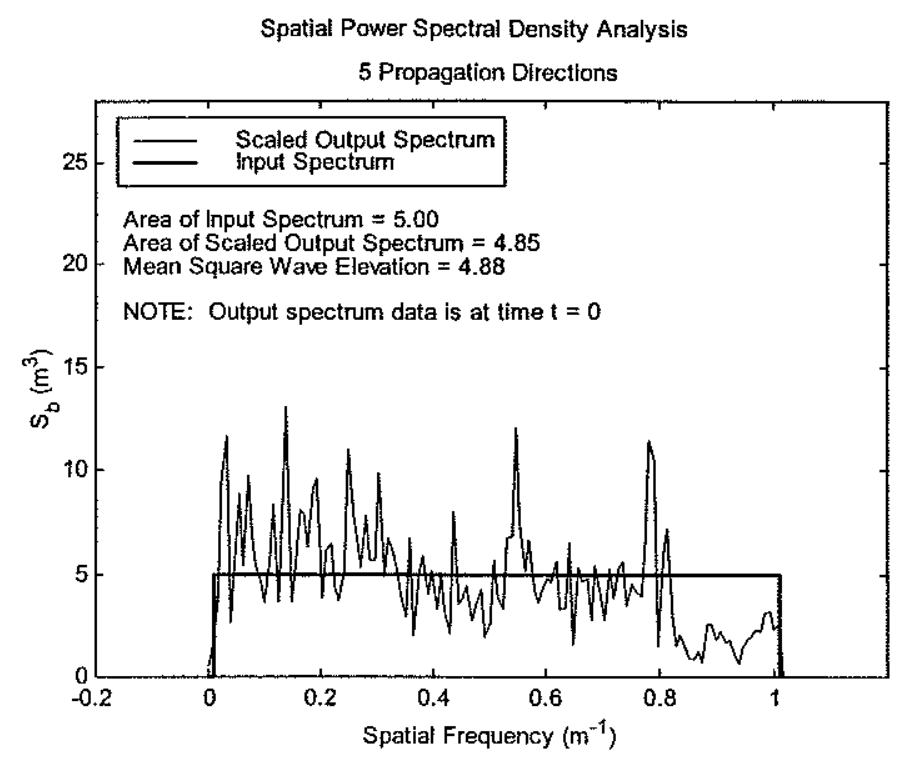

Figure 2-19: Spectral Analysis of Spatial Wave Data - Five Propagation Directions

concept applies to angles in between the extreme cases as a small portion of the wave field is stretched to fit the antenna.

The stretching causes a decrease in spatial frequency as the apparent wavelength of the individual wave is increased, as shown in Figure 2-20. Since the individual spatial frequency decreases, the total energy of the wave field along the antenna shifts to lower frequencies. It should also be pointed out that the shifts in energy magnitude occur at values that correspond to the cosine of the angle between the individual waves and the antenna. In the case of three propagation directions, shown in Figure 2-18, the waves are propagating at $-60^{\circ}, 0^{\circ}$, and $+60^{\circ}$. The shift in energy magnitude occurs at $0.5 \mathrm{~m}^{-1}$, which is the same value as the width of the input spectrum multiplied by $\cos \left(60^{\circ}\right)$. In the case of five propagation directions, shown in Figure $2-19$, the waves are propagating at $-72^{\circ},-36^{\circ}, 0^{\circ},+36^{\circ}$, and $+72^{\circ}$. The shifts in energy magnitude occur at $0.3 \mathrm{~m}^{-1}$ and $0.8 \mathrm{~m}^{-1}$, which are the same values as the width of the input spectrum multiplied by $\cos \left(36^{\circ}\right)$ and $\cos \left(72^{\circ}\right)$, respectively. 


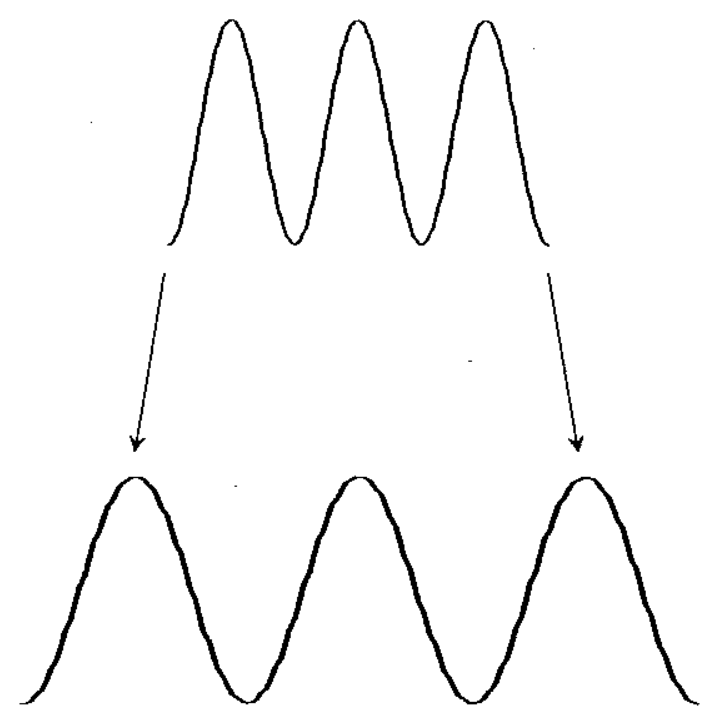

Figure 2-20: Spatial Frequency Stretching

\subsubsection{Temporal Spectral Analysis}

By sampling the wave record temporally for sea surface elevation, one can determine the temporal power spectral density curve of the modeled wave field. By comparing the resultant spectral density curve to the desired spectral density curve, a verification of the model's wave field generation method can be conducted. Figure 2-21 shows the temporal spectral analysis of a model generated wave field. The resultant energy density spectrum closely resembles the desired input spectrum and there is good agreement amongst the values representing the area under the input spectrum, the area under the output spectrum, and the mean square wave elevation of the modeled wave field. 


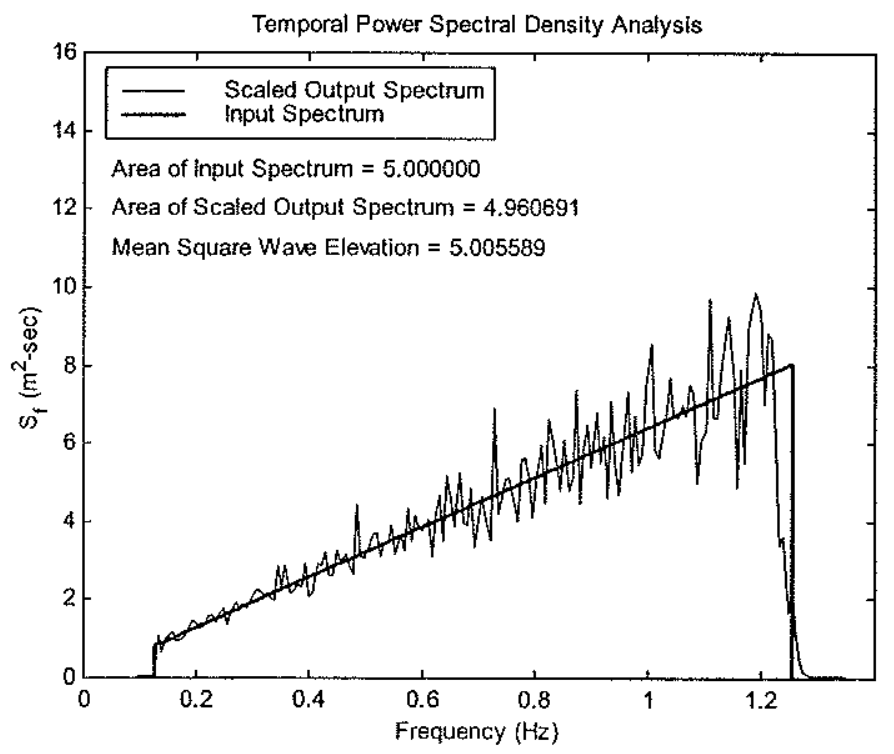

Figure 2-21: Spectral Analysis of Temporal Wave Data 


\section{Chapter 3}

\section{Antenna Model Development}

\subsection{Coordinate System Description}

The coordinate system adopted for the model is a three-dimensional system whose origin translates in the antenna tow direction at the antenna tow speed $U$, which is defined to be positive. The origin of the antenna is defined to be the trailing point of the antenna. The waves travel in the positive $\mathrm{x}$ direction, all at varying angles, but always from left to right in the positive $\mathrm{x}$ direction. Figure 3-1 depicts the coordinate system in the $x-z$ plane as well as the basic antenna configuration. The antenna tow angle, $\theta$, is the angle measured from the antenna to the principal axis of wave propagation. It is defined to be $0^{\circ}$ when pointing in the positive $\mathrm{x}$ direction and $180^{\circ}$ when pointing in the negative $\mathrm{x}$ direction. The model allows the user to select any tow angle between and including $0^{\circ}$ and $180^{\circ}$. Due to port and starboard symmetry of the anntena, the behavior of the antenna for the angles $180^{\circ}$ to $360^{\circ}$ is identical to the behavior of the antenna for the angles $0^{\circ}$ to $180^{\circ}$. Figure 3-2 depicts the coordinate system in the $x-y$ plane as well as the antenna tow direction.

The equations listed in Table 2.1 apply to a stationary coordinate system. The transformation to a moving coordinate system employs the following relationship

$$
x^{\prime}=x+U t
$$

where 


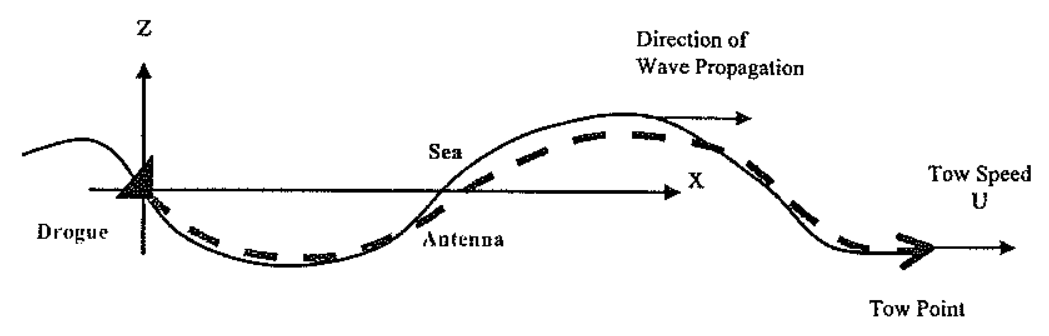

Figure 3-1: Coordinate System: X-Z Plane

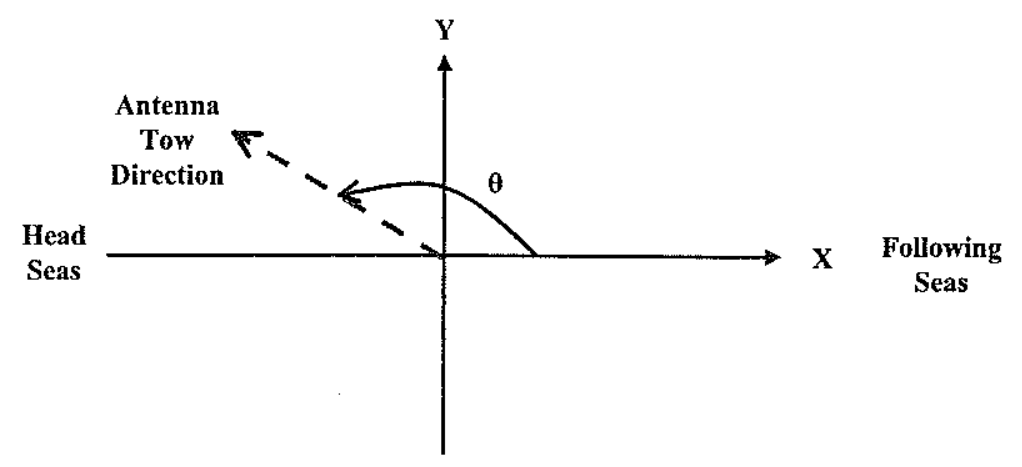

Figure 3-2: Coordinate System: X-Y Plane 
$x^{\prime}$ is the earth-fixed, stationary, coordinate system

$x$ is the moving coordinate system whose origin translates with velocity $U$, as shown in Figures 3-1 and 3-2.

\subsection{Governing Vertical Equation of Motion}

The vertical forces acting on the antenna are given in the right hand side of Equation 3.2

$$
m \frac{d v}{d t}=T(x, t) \frac{\partial^{2} q}{\partial x^{2}}+C_{D}(w-v)|w-v|+b(x, t)+C_{M A} \rho_{w a t e r} \pi r^{2} \frac{d w}{d t}+E I \frac{\partial^{4} q}{\partial x^{4}}
$$

where

$m$ is the mass per unit length of the antenna, assumed uniform

$v$ is antenna vertical velocity, which is equal to $\frac{d q}{d t}$

$T(x, t)$ is the tension along the length of the antenna, arising from skin frictional drag between the submerged portion of the antenna and the water

$q$ is the $\mathrm{z}$ coordinate of the antenna central axis, as shown in Figure 3-3

$C_{D}$ is the normal drag coefficient of a cylindrical antenna section as it moves in the $\mathrm{z}$ direction through the water

$w$ is the fluid vertical velocity

$b(x, t)$ is the net buoyancy force acting on the antenna, which is the difference between the downward force due to its weight and the upward force due to the displaced water.

$C_{M A}$ is the coefficient of added mass for a cylindrical section in a flow field normal to its long axis

$\rho_{\text {water }}$ is the density of water (for seawater of $15^{\circ} \mathrm{C}, \rho_{\text {water }}=1025.9 \mathrm{~kg} / \mathrm{m}^{3}$ )

$r$ is the antenna radius

$E I$ is the product of the antenna cross sectional modulus of elasticity and area moment of inertia, thus a measure of lateral stiffness.

The first term on the right hand side of Equation 3.2 represents a restoring force characteristic of a string displaced from its equilibrium condition. The tension in the antenna is calculated from the time varying wetted surface area along the length of the antenna. A tangential drag coefficient of $C_{T}=0.0035$ was used based upon Reynold's numbers which ranged 


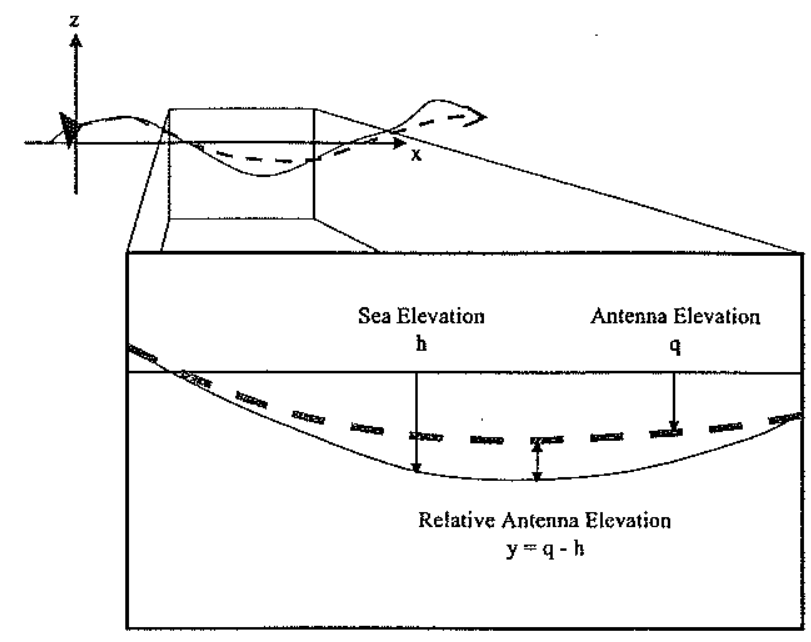

Figure 3-3: Antenna and Sea Surface Elevation Variables

from 32,940 to 592,900 for speed/diameter combinations of 3 knots $/ 1$ inch to 9 knots $/ 6$ inches, respectively. [1] The second term represents the normal drag force a cylindrical element would experience as it is moved through the fluid with its long axis perpendicular to the direction of motion. A value of $C_{D}=1.0$ was used, which assumes an infinite fluid domain. The fourth term represents the added mass effect experienced by a cylindrical object as the fluid around it is accelerating normal to the long axis of the cylinder. A value of $C_{M A}=1.0$ was used, which is consistent with standard fluid dynamic texts assuming an infinite fluid domain. [6] The final term represents the restoring force due to the stiffness of the material, and its distribution with respect to the neutral axis. Initial design options have the lateral stiffness ranging from 300 to $1400 P a-m^{2}$.

It is recognized that some of these forces have been approximated, namely in that the values quoted above are for $C_{D}$ and $C_{M A}$ in an infinite fluid domain. The antenna operates in close proximity to the free surface, and occasionally portions rise completely above the surface of the water. A rigorous treatment of the behavior of $C_{D}$ and $C_{M A}$ near the free surface was not attempted. Rather, a straightforward linear approximation was employed which varied the coefficients $C_{D}$ and $C_{M A}$ from their infinite fluid domain values when the antenna axis was at 

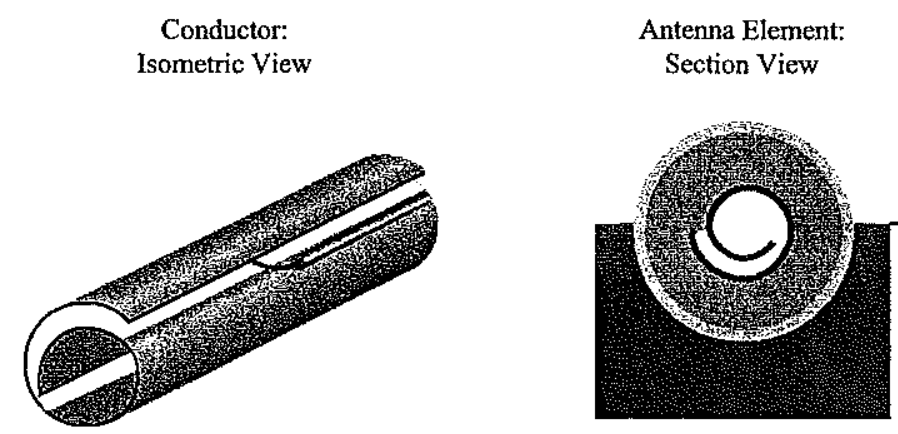

Figure 3-4: Antenna Element Description

a depth of $r$, just submerged, to a value of zero when the antenna axis was at a height of $r$, just breaking out of the water. This dependence of $C_{D}$ and $C_{M A}$ on relative antenna depth, $y$, is shown in Equations 3.3 and 3.4, which only apply to the partially submerged case.

$$
\begin{aligned}
C_{D}(y) & =C_{D_{\text {Infinite }}}\left(-\frac{y}{2 r}+\frac{1}{2}\right) \\
C_{M A}(y) & =C_{M A_{\text {Infinite }}}\left(-\frac{y}{2 r}+\frac{1}{2}\right)
\end{aligned}
$$

\subsection{Antenna Modeling Methods}

\subsubsection{Discretization}

The antenna, of length $L$, is subdivided first into lengths of the physical radio antenna elements. These antenna elements, designed at Lincoln Laboratory, are called "jelly roll" antennas because they are a conducting sheet rolled up about $1 \frac{1}{2}$ times. A graphical description of the antenna element is shown in Figure 3-4. The element length is designated $L_{\text {elem }}$ * This first level of subdivision is not only logical, but necessary for the exposure statistics calculated by the model. 


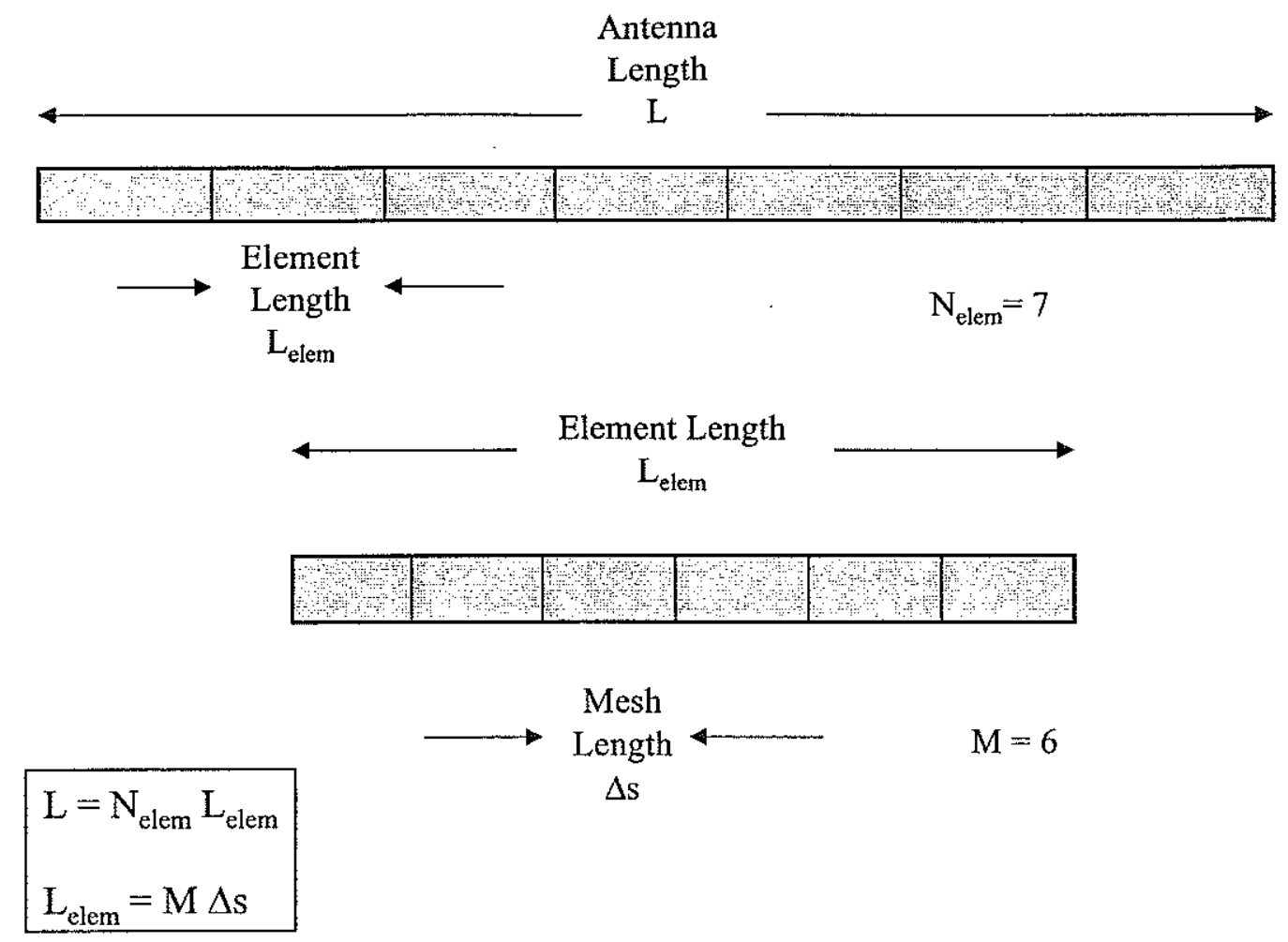

Figure 3-5: Antenna and Antenna Element Description

As it turns out, this type of antenna element performs well as long as it has some small amount, on the order of $1 \mathrm{~mm}$, of its outer housing diameter exposed along its entire length. But if the element becomes covered by even a small amount of water at any point along its length, antenna performance drops dramatically. Thus it is necessary to monitor numerous points within each element to determine whether a given element is exposed or not. This leads to the final level of antenna subdivision: each element is meshed into some integer number $M$ of sections with length $\Delta s$. The subdivision process is shown in Figure 3-5.

An interesting feature of the models which calculate the sea elevation and fluid velocities by Fast Fourier Transforms (FFTs) is that the frequency meshing $(\Delta \omega)$ of the spectral energy density function $S(\omega)$, is now related to the spatial meshing $(\Delta x)$ of the ${ }_{i}$ sea, and thus the spatial meshing of the antenna $(\Delta s)$. This means that there is an extra constraint to consider 
in selecting the finest antenna mesh size $\Delta s$; or conversely, given a mesh size $\Delta s$, constraints are imposed on the $\Delta \omega$ meshing of the spectral energy density function $S(\omega)$. Some freedom is regained if one decouples the length of the antenna and the length of the sea simulated. This decoupling is implemented in the model, so that for a typical run the antenna may be $25 \mathrm{~m}$ long with a mesh size of $0.0625 \mathrm{~m}$, while the length of sea modeled is $512 \mathrm{~m}$ long with a mesh size of $0.0625 \mathrm{~m}$. The key point is that the mesh length between the antenna $(\Delta s)$ and the sea $(\Delta x)$ must match.

\subsubsection{Integration Method}

The time marching integration method implemented is an explicit forward Euler scheme. The following discussion of of the explicit forward Euler scheme comes from Ulrich. [9] Such schemes are characterized by using information at time $t$ to calculate functions at time $t+\Delta t$. A simple example of how the forward Euler method is used is given below. Consider the following sample numerical problem.

Solve

$$
\begin{aligned}
\frac{d u}{d t} & =u^{2} \\
u(0) & =1
\end{aligned}
$$

The time derivative is approximated using tho Euler method as

$$
\left.\frac{d u}{d t}\right|_{t=t^{n}}=\frac{U^{n+1}-U^{n}}{t^{n+1}-t^{n}}
$$

which for constant time step $\Delta t$ becomes

$$
\left.\frac{d u}{d t}\right|_{t=t^{n}}=\frac{U^{n+1}-U^{n}}{\Delta t}
$$

having adopted the notation of Celia and Gray [2], where

$$
U^{n} \equiv u\left(t^{n}\right)
$$


and $t^{n}$ is the $n^{t h}$ time step. Then the solution proceeds step by step, beginning with the initial value of $u$ given in Equation 3.6

$$
\left.\frac{d u}{d t}\right|_{t=0}=u(0)^{2}=1
$$

then solving Equation 3.8 for $U^{n+1}$ we obtain

$$
U^{1}=U^{0}+\left.\Delta t \frac{d u}{d t}\right|_{t=0}
$$

With the value of $u$ now known at time $t\left(U^{1}\right)$, we can calculate

$$
\left.\frac{d u}{d t}\right|_{t=1}=\left(U^{1}\right)^{2}
$$

and

$$
U^{2}=U^{1}+\left.\Delta t \frac{d u}{d t}\right|_{t=t^{1}}
$$

In like manner, we move forward in time to calculate any desired $U^{n}$. Numerical experimentation was used to determine the appropriate time step size which produced acceptable results.

The spatial derivatives $\left(\frac{d^{n} q}{d x^{n}}\right)$ used in the model are classified as centered finite-divideddifference formulas. [3] Thus the antenna algorithm can be classified as a forward time, centered space (FTCS) method.

\subsubsection{Boundary Conditions}

The boundary conditions imposed on the antenna endpoints are given in Equations 3.14 and 3.15 as

$$
\begin{aligned}
& q(0)=h(0) \\
& q(L)=h(L)
\end{aligned}
$$


That is, the antenna follows the sea surface at its endpoints. This is envisioned to be accomplished at the trailing end by a drogue having positive buoyancy, and at the tow point by one of several options. These options include

- a dynamic lifting body

- a surface following buoy

- a leader of positive buoyancy

- some combination of the above features.

\subsubsection{Initial Conditions}

The antenna simulation begins at $t=0$ with

$$
\begin{aligned}
q(x) & =h(x) \\
\frac{d q(x)}{d t} & =\frac{d h(x)}{d t}
\end{aligned}
$$

that is, the antenna is placed at the sea surface, and given an initial vertical velocity matching that of the sea surface. 


\section{Chapter 4}

\section{Modeling Results}

\subsection{Exposure Statistics}

\subsubsection{Definition of Exposure}

The primary goal of the modeling effort was to predict exposure statistics as various design and operational parameters were varied; therefore, it is necessary to define exposure before proceeding on to the exposure statistics results. The overall exposure of the antenna is driven by the exposure of individual antenna elements, so the determination of an exposed, or receiving, antenna element must be addressed. The experimentally observed performance characteristics of the "jelly roll" antenna elements led to the following definition of exposure. It was found that for a level element in calm water, the antenna gain is stable right up to the point at which the outer skin becomes completely submerged. It was also found that if any portion of the outer skin was completely covered by water then the gain of the element suffered drastically. Thus an exposed element was defined to be one which was exposed by some small threshold height, set to four percent of Diameter for all simulations, at every point along the length of the element. In a discrete computer model some approximation is necessary with regard to every point along the length of the element. Thus each antenna element was divided into smaller segments for analysis. Typical designs considered had an element length of $0.5 \mathrm{~m}$ and were further divided, or oversampled, at $M=8$. 


\subsubsection{Statistics Calculated by the Model}

The computer model was programmed to track three key statistics:

- average exposure - the average fraction of antenna elements exposed throughout the entire duration of the simulation

- threshold met - the average fraction of the time that some specified number of antenna elements, or more, were exposed

- average counterdetection length - the average length of the antenna where there is no water touching the antenna.

In addition, a record is made at each sample time of the condition of every antenna element, such that when the simulation is finished, an exposure matrix is available for further postprocessing. Examples of information which could be extracted from the exposure matrix include

- average drop-out time of a specific element

- average spatial length of wave wash-overs

- histograms and standard deviations of the number of exposed elements

- exposure statistics for an array not fully populated (i.e., one could examine only the columns which had antenna elements installed) .

Five output files are generated for each run of the computer model. These output files are described in detail in Appendix A. The input parameters which are specified for each run of the computer model are also described in detail in Appendix A.

In the exposure statistics results given below, each series of simulations is presented in the following manner:

- a listing of the antenna and operational parameters input table used to generate the series

- a plot of the average fraction of elements exposed 
- a brief discussion of the exposure plot.

It is important to realize that these plots represent averages in time and space. This is necessary in order to display the results as functions of key antenna design and operational parameters, which is most useful from a designer's viewpoint. From the output files, it is also possible to analyze how the statistics vary as time progresses, or how the statistics vary along the array from the tow point to the trailing end. Although there are a large number of possible statistics that could be analyzed for an even larger number of design and operational parameter combinations, only one statistic is presented here for seven selected design or operational parameters. 


\begin{tabular}{ll}
\hline Parameter & Description \\
\hline \hline Duration & $60 \mathrm{sec}$ \\
$N_{\text {elem }}$ & 73 \\
$L_{\text {ant }}$ & $36.5 \mathrm{~m}$ \\
$L_{\text {elem }}$ & $0.5 \mathrm{~m}$ \\
Diameter & 3.0 inches \\
EI & $100 \mathrm{~N}-\mathrm{m}^{2}$ \\
$\rho_{\text {ant }}$ & $408 \mathrm{~kg} / \mathrm{m}^{3}$ \\
$M$ & 8 \\
$U$ & $5.0 \mathrm{kts}$ \\
Tow Angle & $0^{\circ}, 45^{\circ}, 90^{\circ}, 135^{\circ}, 180^{\circ}$ \\
$H_{s}$ & $3.0^{\mathrm{m}}$ \\
Development & 1.0 \\
Spectral Model & $\mathrm{Bretschneider}$ \\
Spreading Function & $\cos ^{2}$ \\
$N_{\text {freq }}$ & $2^{13}$ \\
NN & $1,5,9,13,17,21$ \\
\hline
\end{tabular}

Table 4.1: Series 1 Input Parameters

\subsection{Results}

\subsubsection{Series 1: Statistics vs. Number of Propagation Directions}

\section{Input}

The input parameters for Series 1: Statistics versus Number of Propagation Directions, are given in Table 4.1. This is a measure of the fineness of the angular sea description. The number of propagation directions used were $1,5,9,13,17$, and 21 . The tow angles used were $0,45,90,135$, and 180 degrees. 


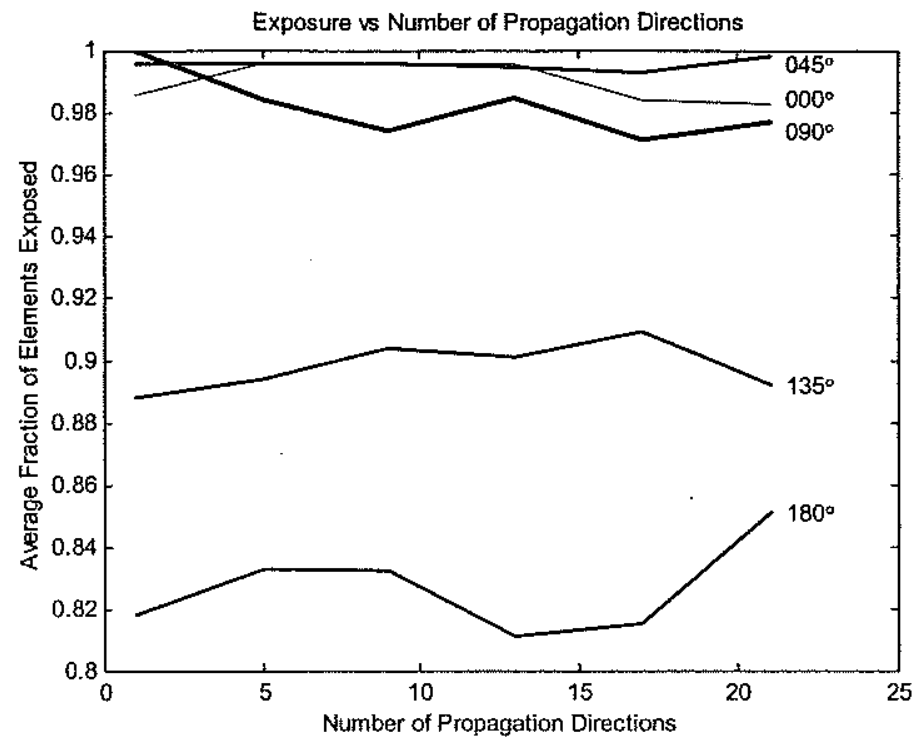

Figure 4-1: Average Exposure vs. Number of Propagation Directions

\section{Results}

Figure 4-1 shows a very weak dependence of the average fraction of elements exposed on the number of propagation directions used by the model to simulate the multidirectional wave field. However, the plot shows a strong dependence of average exposure on tow angle. The insensitivity to the number of propagation directions in the sea description not only demonstrates the validity of the approach used, but also shows that computing time can be minimized by using just a modest number ( 3 to 9 ) of directions in the sea description. 


\begin{tabular}{ll}
\hline Parameter & Description \\
\hline \hline Duration & $60 \mathrm{sec}$ \\
$N_{\text {elem }}$ & 73 \\
$L_{\text {ant }}$ & $36.5 \mathrm{~m}$ \\
$L_{\text {elem }}$ & $0.5 \mathrm{~m}$ \\
Diameter & $3.0 \mathrm{inches}$ \\
EI & $100 \mathrm{~N}-\mathrm{m}^{2}$ \\
$\rho_{\text {ant }}$ & $408 \mathrm{~kg} / \mathrm{m}^{3}$ \\
$M$ & 8 \\
$U$ & $5.0 \mathrm{kts}$ \\
Tow Angle & $0^{\circ}, 30^{\circ}, 60^{\circ}, 90^{\circ}, 120^{\circ}, 150^{\circ}, 180^{\circ}$ \\
$H_{s}$ & $3.0 \mathrm{~m}$ \\
Development & 1.0 \\
Spectral Model & $\mathrm{Bretschneider}$ \\
Spreading Function & $\cos ^{2}$ \\
$N_{\text {freq }}$ & $2^{13}$ \\
NN & 3 \\
\hline
\end{tabular}

Table 4.2: Series 2 Input Parameters

\subsubsection{Series 2: Statistics vs. Tow Angle}

\section{Input}

The input parameters for Series 2: Statistics versus Tow Angle, are given in Table 4.2. The tow angles used were $0,30,60,90,120,150$, and 180 degrees. 


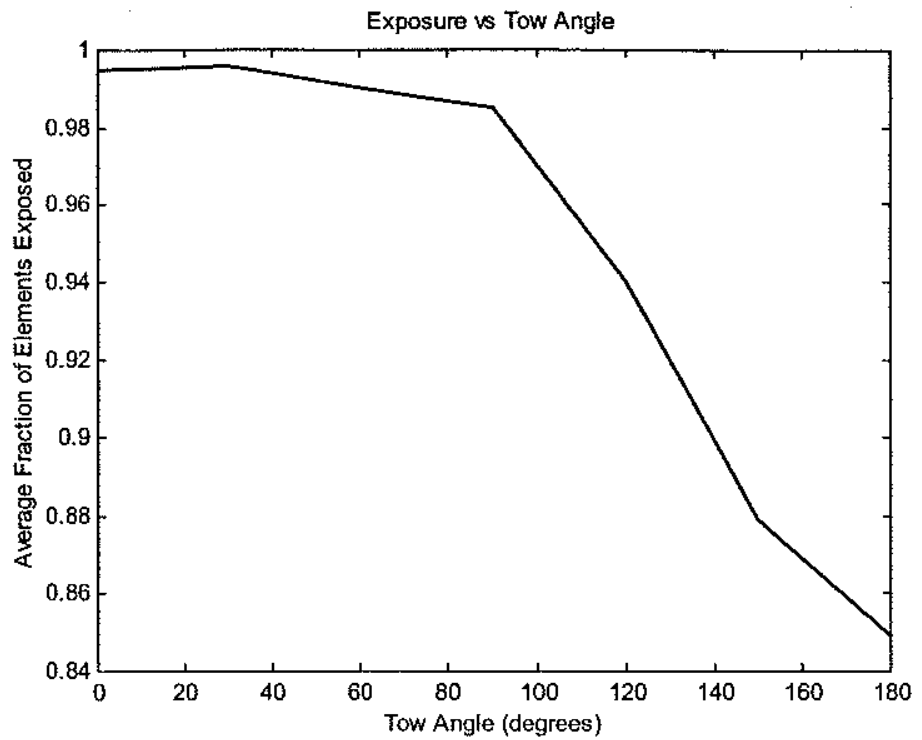

Figure 4-2: Average Exposure vs. Tow Angle

\section{Results}

Figure 4-2 shows a rather weak dependence of the average fraction of elements exposed on the tow angle from $0^{\circ}-90^{\circ}$ (following seas); however, there is a very strong dependence on tow angle from $90^{\circ}-180^{\circ}$ (head seas). 


\begin{tabular}{ll}
\hline Parameter & Description \\
\hline \hline Duration & $60 \mathrm{sec}$ \\
$N_{\text {elem }}$ & 73 \\
$L_{\text {ant }}$ & $36.5 \mathrm{~m}$ \\
$L_{\text {elem }}$ & $0.5 \mathrm{~m}$ \\
Diameter & $3.0 \mathrm{inches}$ \\
EI & $100 \mathrm{~N}-\mathrm{m}^{2}$ \\
$\rho_{\text {ant }}$ & $408 \mathrm{~kg} / \mathrm{m}^{3}$ \\
$M$ & 8 \\
$U$ & $5.0 \mathrm{kts}$ \\
Tow Angle & $0^{\circ}, 45^{\circ}, 90^{\circ}, 135^{\circ}, 180^{\circ}$ \\
$H_{s}$ & $0.5,1.0,1.5,2.0,2.5,3.0 \mathrm{~m}$ \\
Development & 1.0 \\
Spectral Model & $\mathrm{Bretschneider}$ \\
Spreading Function & $\cos ^{2}$ \\
$N_{\text {freq }}$ & $2^{13}$ \\
NN & 3 \\
\hline
\end{tabular}

Table 4.3: Series 3 Input Parameters

\subsubsection{Series 3: Statistics vs. Sea Severity}

\section{Input}

The input parameters for Series 3: Statistics versus Sea Severity, are given in Table 4.3. The significant wave heights used were $0.5,1.0,1.5,2.0,2.5$, and 3.0 meters. The tow angles used were $0,45,90,135$, and 180 degrees. 


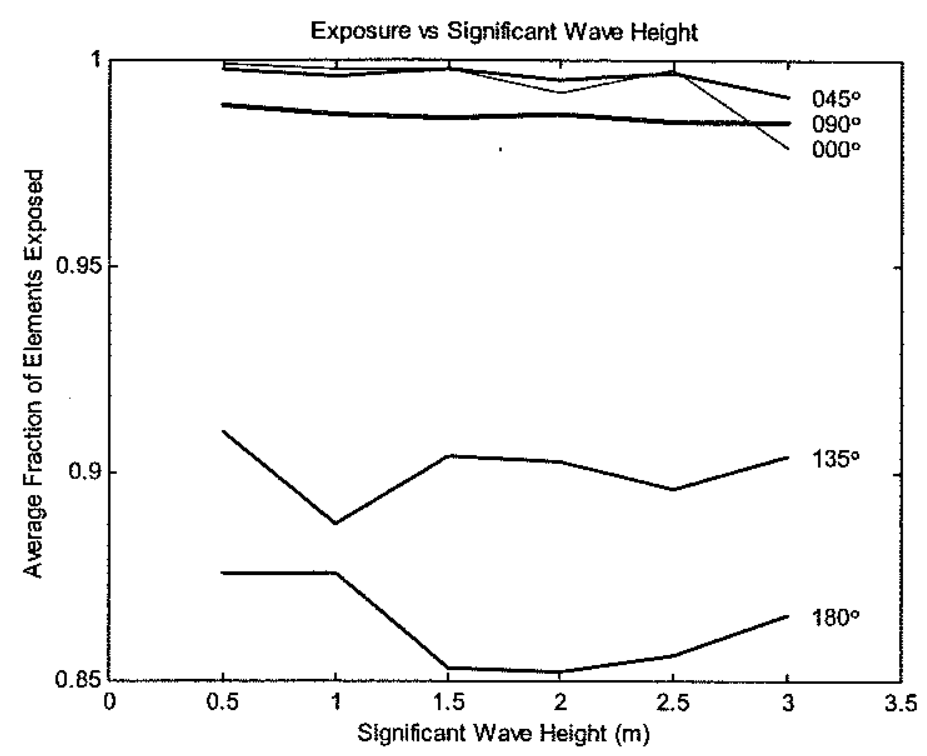

Figure 4-3: Average Exposure vs. Sea Severity

\section{Results}

Figure 4-3 shows a rather weak dependence of the average fraction of elements exposed on the significant wave height at all tow angles. 


\begin{tabular}{ll}
\hline Parameter & Description \\
\hline \hline Duration & $60 \mathrm{sec}$ \\
$N_{\text {elem }}$ & 73 \\
$L_{\text {ant }}$ & $36.5 \mathrm{~m}$ \\
$L_{\text {elem }}$ & $0.5 \mathrm{~m}$ \\
Diameter & 3.0 inches \\
EI & $200 \mathrm{~N}-\mathrm{m}^{2}$ \\
$\rho_{\text {ant }}$ & $408 \mathrm{~kg} / \mathrm{m}^{3}$ \\
$M$ & 8 \\
$U$ & $3,5,7,9 \mathrm{kts}$ \\
Tow Angle & $0^{\circ}, 45^{\circ}, 90^{\circ}, 135^{\circ}, 180^{\circ}$ \\
$H_{s}$ & $3.0 \mathrm{~m}$ \\
Development & 1.0 \\
Spectral Model & $\mathrm{Bretschneider}_{\text {Spreading Function }} \cos ^{2}$ \\
$N_{\text {freq }}$ & $2^{13}$ \\
NN & 3 \\
\hline
\end{tabular}

Table 4.4: Series 4 Input Parameters

\subsubsection{Series 4: Statistics vs. Tow Speed}

\section{Input}

The input parameters for Series 4: Statistics versus Tow Speed, are given in Table 4.4. The tow speeds used were $3,5,7$, and 9 knots. The tow angles used were $0,45,90,135$, and 180 degrees. 


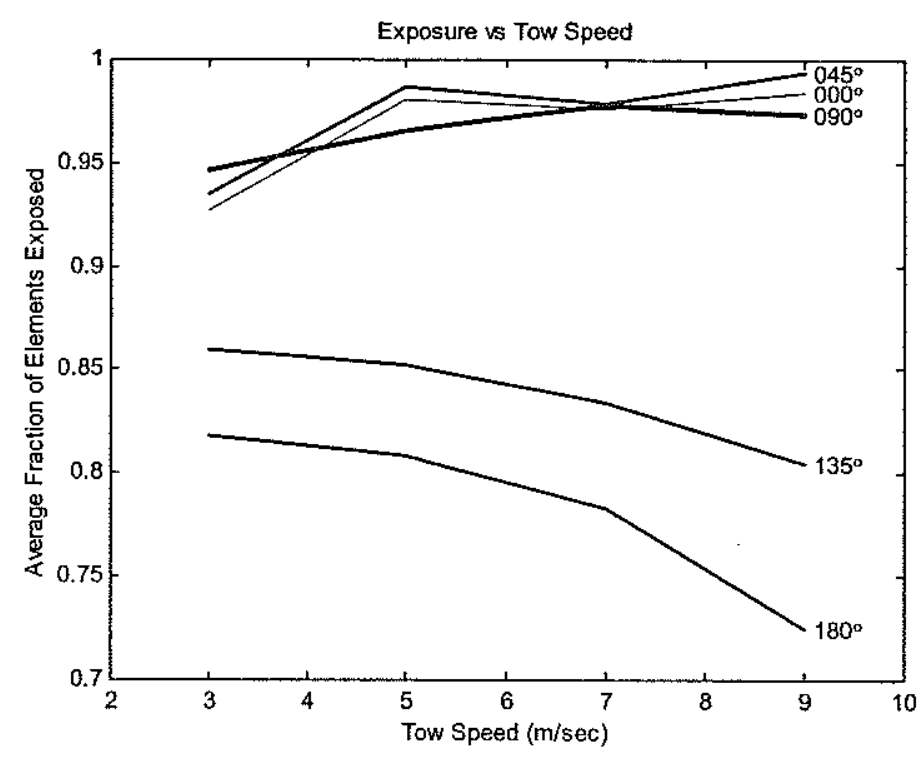

Figure 4-4: Average Exposure vs. Tow Speed

\section{Results}

Figure 4-4 demonstrates the effect that tow angle has on other parameters. In this instance, increasing tow speed for tow angles greater than $90^{\circ}$ (head seas) causes a decrease in exposure fraction. While, for tow angles less than $90^{\circ}$ (following seas), increasing tow speed causes an increase in exposure fraction. 


\begin{tabular}{ll}
\hline Parameter & Description \\
\hline Duration & $60 \mathrm{sec}$ \\
$N_{\text {elem }}$ & $10,30,50,120,200$ \\
$L_{\text {ant }}$ & $5,15,25,60,100 \mathrm{~m}$ \\
$L_{\text {elem }}$ & $0.5 \mathrm{~m}$ \\
Diameter & 3.0 inches \\
EI & $200 \mathrm{~N}-\mathrm{m}^{2}$ \\
$\rho_{\text {ant }}$ & $408 \mathrm{~kg} / \mathrm{m}^{3}$ \\
$M$ & 8 \\
$U$ & $5.0 \mathrm{kts}$ \\
Tow Angle & $0^{\circ}, 45^{\circ}, 90^{\circ}, 135^{\circ}, 180^{\circ}$ \\
$H_{s}$ & $3.0 \mathrm{~m}$ \\
Development & 1.0 \\
Spectral Model & $\mathrm{Bretschneider}$ \\
Spreading Function & $\cos ^{2}$ \\
$N_{\text {freq }}$ & $2^{13}$ \\
NN & 3 \\
\hline
\end{tabular}

Table 4.5: Series 5 Input Parameters

\subsubsection{Series 5: Statistics vs. Antenna Length}

\section{Input}

The input parameters for Series 5: Statistics versus Antenna Length, are given in Table 4.5. The antenna lengths used were $5,15,25,60$, and 100 meters. The tow angles used were 0,45 , 90,135 , and 180 degrees. 


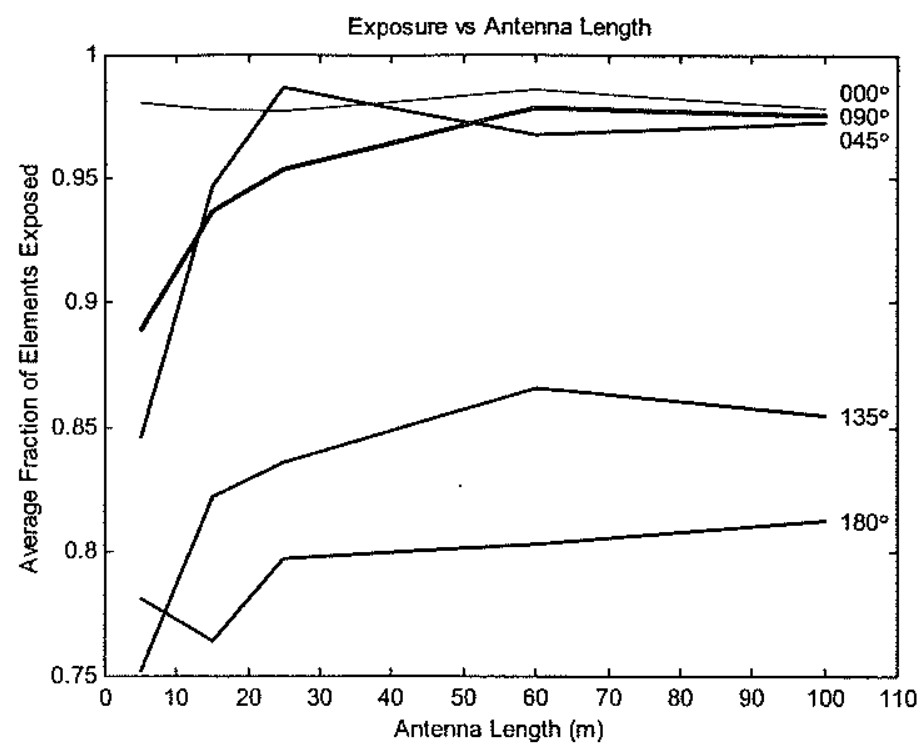

Figure 4-5: Average Exposure vs. Antenna Length

\section{Results}

Figure 4-5 shows a very strong dependence of the average fraction of elements exposed on the antenna length for lengths less than 25 meters. For lengths greater than 25 meters, there is little or no dependence on antenna length. 


\begin{tabular}{ll}
\hline Parameter & Description \\
\hline \hline Duration & $60 \mathrm{sec}$ \\
$N_{\text {elem }}$ & 73 \\
$L_{\text {ant }}$ & $36.5 \mathrm{~m}$ \\
$L_{\text {elem }}$ & $0.5 \mathrm{~m}$ \\
Diameter & $3.0,6.0$ inches \\
EI & $200 \mathrm{~N}-\mathrm{m}^{2}$ \\
$\rho_{\text {ant }}$ & $408 \mathrm{~kg} / \mathrm{m}^{3}$ \\
$M$ & 8 \\
$U$ & $5.0 \mathrm{kts}$ \\
Tow Angle & $0^{\circ}, 45^{\circ}, 90^{\circ}, 135^{\circ}, 180^{\circ}$ \\
$H_{s}$ & $3.0 \mathrm{~m}$ \\
Development & 1.0 \\
Spectral Model & $\mathrm{Bretschneider}$ \\
Spreading Function & $\cos ^{2}$ \\
$N_{\text {freq }}$ & $2^{13}$ \\
NN & 3 \\
\hline
\end{tabular}

Table 4.6: Series 6 Input Parameters

\subsubsection{Series 6: Statistics vs. Antenna Diameter}

\section{Input}

The input parameters for Series 6: Statistics versus Antenna Diameter, are given in Table 4.6. The antenna diameters used were 3 and 6 inches. The tow angles used were $0,45,90,135$, and 180 degrees. 


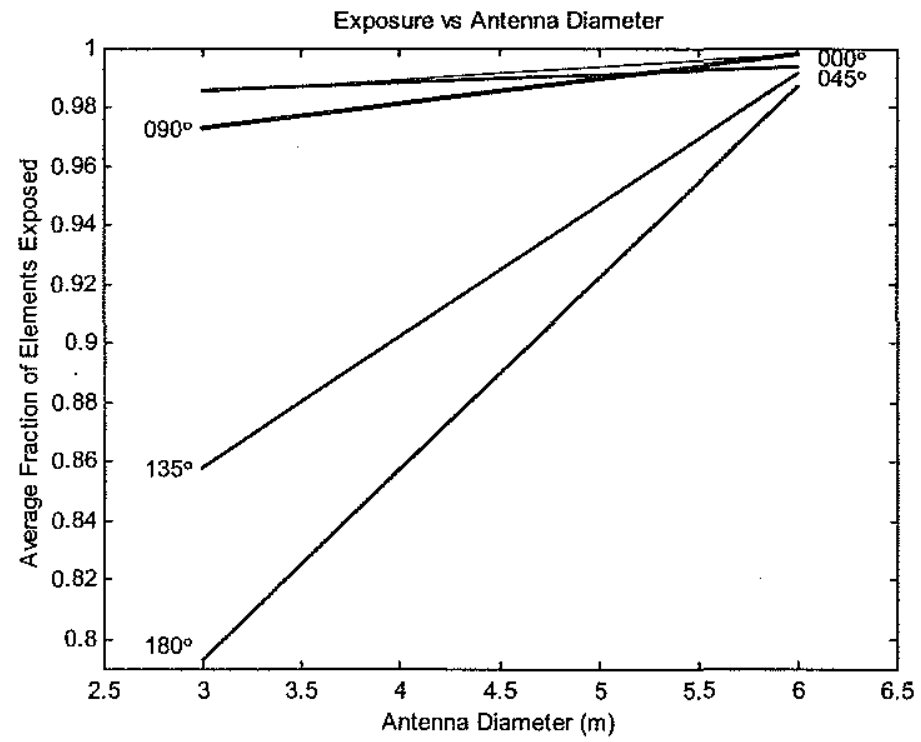

Figure 4-6: Average Exposure vs. Antenna Diameter

\section{Results}

Figure 4-6 also demonstrates the influence that tow angle has on amplifying the effects of design parameter changes. In all cases shown, the larger diameter antenna has an increased exposure fraction, but the change is most significant for head seas cases. 


\begin{tabular}{ll}
\hline Parameter & Description \\
\hline \hline Duration & $60 \mathrm{sec}$ \\
$N_{\text {elem }}$ & 73 \\
$L_{\text {ant }}$ & $36.5 \mathrm{~m}$ \\
$L_{\text {elem }}$ & $0.5 \mathrm{~m}$ \\
Diameter & 3.0 inches \\
EI & $200 \mathrm{~N}-\mathrm{m}^{2}$ \\
$\rho_{\text {ant }}$ & $200,300,400,500,600 \mathrm{~kg} / \mathrm{m}^{3}$ \\
$M$ & 8 \\
$U$ & $5.0 \mathrm{kts}$ \\
Tow Angle & $0^{\circ}, 45^{\circ}, 90^{\circ}, 135^{\circ}, 180^{\circ}$ \\
$H_{s}$ & $3.0 \mathrm{~m}$ \\
Development & 1.0 \\
Spectral Model & $\mathrm{Bretschneider}$ \\
Spreading Function & $\cos ^{2}$ \\
$N_{\text {freq }}$ & $2^{13}$ \\
NN & 3 \\
\hline
\end{tabular}

Table 4.7: Series 7 Input Parameters

\subsubsection{Series 7: Statistics vs. Antenna Density}

\section{Input}

The input parameters for Series 7: Statistics versus Antenna Density, are given in Table 4.7.

The antenna densities used were $200,300,400,500$, and $600 \mathrm{~kg} / \mathrm{m}^{3}$. The tow angles used were $0,45,90,135$, and 180 degrees. 


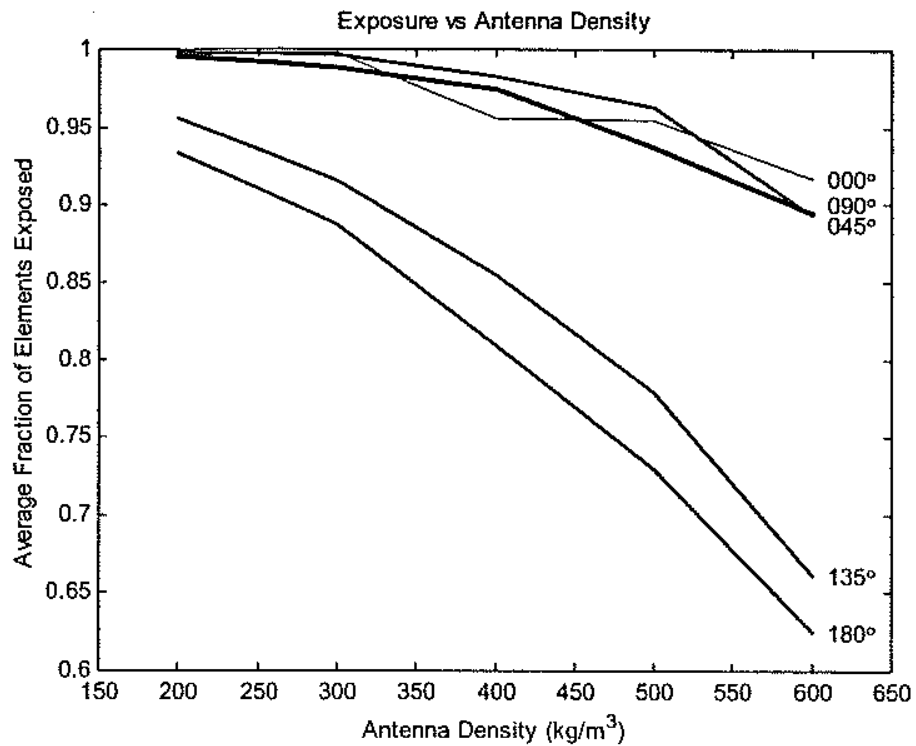

Figure 4-7: Average Exposure vs. Antenna Density

\section{Results}

Figure 4-7 again demonstrates the amplifying effect that tow angle has on design parameter changes. In all cases shown, as you increase the antenna density there is a resulting decrease in exposure fraction; however, this decrease is amplified for tow angles in the head seas direction. 


\section{Chapter 5}

\section{Future Efforts}

The combined Navy and DARPA Multi-element Buoyant Cable Antenna effort was funded in FY 00 for 3 years. The demonstration phase culminated with a successful testing effort in Hawaii in the Summer of 1999. Within FY 00, Lincoln Laboratory is scheduled to complete the development and design of an antenna which can stand up to the rigors of an at sea deployment. FY 01 will see the initial production of the deployable version with the goal of at sea submarine testing during FY 02 .

Work at MIT will continue to support the buoyant cable antenna effort. Another graduate student, under the supervision of Professor Jerome Milgram, will continue and extend the scope of the simulation effort. Areas which will be investigated to improve the capability of the computer model include

- more detailed modeling of the hydrodynamics at the free surface

- higher order integration schemes

- addition of digital signal processors to improve computation speed

- validation against tow tank data

- validation against at-sea test data

- effects of roll for revised antenna elements whose performance depends on roll angle. 


\section{Appendix A}

\section{User's Guide to Proteus}

\section{A.1 Introduction}

\section{A.1.1 Contents of the User's Guide}

The User's Guide is broken down into three sections:

- Introduction

- Input File

- Output Files.

Section A.1 describes the contents of the user's guide and contains the procedures necessary to obtain the results discussed in Section A.3. Section A.2 describes the Proteus input file line by line and includes program features and limitations associated with each parameter. Section A.3 briefly describes the contents of each of the five output files and the recommended methods of viewing or extracting the information.

\section{A.1.2 Analysis Procedure}

\section{Obtaining the Snapshot Output}

1. Ensure that the input file, proteus_input.txt, is located in the same directory as the FORTRAN executable file, proteus.exe. 
2. Modify the input file to reflect the conditions that the user wishes to analyze, using Section A.2 as guidance for features and limitations. Ensure that the matlab_output variable is set to .TRUE.

3. Execute proteus.exe.

4. The snapshot output file (run_id//'_mat') will be located in the same directory as the executable file.

5. Ensure that the output file (run_id//'_mat') will be located in the same directory as the Matlab ${ }^{\circledR}$ function file, proteus mat.

6. At the Matlab ${ }^{\circledR}$ command line type: clear; proteus_mat('run_id//_mat').

\section{Obtaining the Parameters and Results Summary}

1. Ensure that the input file, proteus_input.txt, is located in the same directory as the FORTRAN executable file, proteus.exe.

2. Modify the input file to reflect the conditions that the user wishes to analyze, using Section A.2 as guidance for features and limitations. Ensure that the run_info variable is set to .TRUE.

3. Execute proteus.exe.

4. The parameters and results summary output file (run_id//'_info.txt') will be located in the same directory as the executable file.

5. The output file can be viewed with any standard text editor.

\section{Obtaining the Exposure Data Matrix}

1. Ensure that the input file, proteus input.txt, is located in the same directory as the FORTRAN executable file, proteus.exe.

2. Modify the input file to reflect the conditions that the user wishes to analyze, using Section A.2 as guidance for features and limitations. Ensure that the exp_data variable is set to .TRUE. 
3. Execute proteus.exe.

4. The exposure data matrix output file ( $r u n_{-} i d / /{ }_{-}$edm') will be located in the same directory as the executable file.

5. The output file can be viewed with a user supplied Matlab ${ }^{\circledR}$ file or with a spreadsheet application.

\section{Obtaining Wave Data Temporal Spectrum Analysis}

1. Ensure that the input file, proteus input.txt, is located in the same directory as the FORTRAN executable file, proteus.exe.

2. Modify the input file to reflect the conditions that the user wishes to analyze, using Section A.2 as guidance for features and limitations. Ensure that the wave_temporal variable is set to .TRUE.

3. Execute proteus.exe.

4. The temporal wave data output file ( $r u n_{-} i d / /$ '_- mat2') will be located in the same directory as the executable file.

5. Ensure that the output file (run_id//'_mat2') will be located in the same directory as the executable file, proteus_mat2.exe.

6. Execute proteus_mat2.exe. When the program prompts the user for filename, enter run_id//_mat2.

7. Ensure that the output file (run_id//'_mat2_proc') will be located in the same directory as the Matlab ${ }^{\circledR}$ function file, proteus_mat:.

8. At the Matlab ${ }^{\circledR}$ command line type: clear; proteus_mat2('run_id//_mat2_proc').

\section{Obtaining Wave Data Spatial Spectrum Analysis}

1. Ensure that the input file, proteus_input.txt, is located in the same directory as the FORTRAN executable file, proteus.exe. 
2. Modify the input file to reflect the conditions that the user wishes to analyze, using Section A.2 as guidance for features and limitations. Ensure that the wave_spatial variable is set to .TRUE.

3. Execute proteus.exe.

4. The spatial wave data output file ( $r u n_{-} i d / /$ '_mat3') will be located in the same directory as the executable file.

5. Ensure that the output file (run_id//'_mat3') will be located in the same directory as the executable file, proteus_mat3.exe.

6. Execute proteus_mat3.exe. When the program prompts the user for filename, enter run_id//_mat3.

7. Ensure that the output file (run_id//'_mat3_proc') will be located in the same directory as the Matlab ${ }^{\circledR}$ function file, proteus_mat3.

8. At the Matlab ${ }^{\circledR}$ command line type: clear; proteus_mat3('run_id//_mat3_proc').

\section{A.2 Proteus Input File}

\section{A.2.1 Description of Features and Limitations}

\section{Header}

The input file for Proteus is shown in Section A.2.2. The first 7 lines are for informational purposes and have no effect on the program. However, note that all variables are given in SI units and that there must be 7 lines prior to the first variable defined.

\section{Run Identifier (run_id)}

The run_id is an 8 character variable used to name the 5 output files. The 5 output files are named

- run_id//'_mat' 
- run_id//'_info.txt'

- run_id//'_edm'

- run_id//'_mat2'

- run_id//'_mat3'.

(The // represents FORTRAN90 string concatenation.) Output files are located in the local directory from which the program is run. A sample pattern for run_id is run_id='15May_01'. Characters which Matlab ${ }^{\circledR}$ would interpret as operators $(/,+,-, *$, etc.) should be avoided in run_id if one of the Matlab ${ }^{\circledR}$ output options is selected.

\section{FFT Power of $2(n n f)$}

The FFT algorithm is designed to operate on arrays which have an integer power of 2 elements. $n n f$ sets this power of 2 , making

$$
N \_s e a=2^{n n f}
$$

where

$N \_s e a$ is the number of sea frequency components and spatial locations.

The length of the sea surface modeled in the spatial domain is generally much larger than the antenna length requires; however, the speed advantage of the FFT method more than outweighs the penalty of carrying along the extra sea information.

\section{Number of Antenna Elements (num_of_elem)}

This is the number of physical antenna receiver elements in the array, assuming that

- the array is fully populated

- the elements have zero space between them.

\section{Antenna Element Length (element_length)}

This is the length of each antenna element. 


\section{Antenna Oversample Factor $(M)$}

Each antenna element is broken down into an integer number, $M$, of sections. These sections correspond to the antenna spatial mesh size $\Delta s$. By enforcing the condition that $\Delta s=\Delta x$, the computer model constructs the spatial frequency meshing, $\Delta b$, such that

$$
\begin{aligned}
b_{\max } & =\frac{1}{2 \Delta s} \\
L_{-} \text {sea } & =\Delta s N_{-} \text {sea } \\
\Delta b & =\frac{1}{L_{-} \text {sea }}
\end{aligned}
$$

By invoking the dispersion relation, we convert from the $b$ domain to the $\omega$ domain, using

$$
\omega=\sqrt{2 \pi g b}
$$

This process shows how the spatial meshing relates to the sea energy frequency domain meshing. As one can see, the fact that $N_{-}$sea is constrained to be a power of 2 introduces some limitations on the mesh size $\Delta s$, since it is required that

$$
\text { element_length }=M \Delta s
$$

\section{Tow Speed $(U)$}

The tow speed of the antenna is assumed constant with respect to time. Tow speed must be greater than zero (in order to generate tension). When $\theta$, the angle between an individual propagation direction and the antenna tow angle, is less than 90 degrees (i.e. following seas), sea frequency components above omega_crit,

$$
\text { omega_crit }=\frac{g}{U \cos \theta}
$$

are zeroed out to prevent antenna folding. If omega_crit is less than $1.5 \mathrm{rad} / \mathrm{sec}$, then a warning is issued that excessive frequency components are being zeroed out, and program execution stops. 


\section{Simulation Time (duration)}

The total length of time simulated by the program.

\section{Antenna Time Step Increment $\left(t_{-}\right.$incr $)$}

The time step increment used in solving the antenna's governing equation. A typical value for an antenna with no stiffness is 0.001 seconds. When stiffness is added, the time increment should be reduced to 0.0001 seconds. In all cases, if the program stops executing due to a run-time math error, the time increment should be made smaller to allow proper execution of the program.

\section{Snapshot Output Interval (pic_time)}

This variable allows the user to control the time interval between snapshots, which are recorded to file run_id//'_mat'. These snapshots record both the sea and antenna elevation data along the entire length of the antenna, at a resolution of $\Delta s$.

\section{Time of First Snapshot $\left(t_{-}\right.$first_pic)}

The time to begin recording snapshots is user defined. This is useful to ignore transient behavior at the start of the simulation, or to save only a representative sample at the end of a lengthy simulation.

\section{Statistics Output Interval (stat_time)}

This variable allows the user to control the time interval between exposure data matrix records, which are recorded to file run_id//'_edm'. Selection of an excessively small stat_time results in subsequent records containing essentially the same information, and contributes little to the statistics of the sample.

\section{Snapshot Output Option(matlab output)}

The snapshot output file (run_id//'_mat') is optional, since it does not contain any unique statistical information, and requires a relatively large amount of disk space. The primary 
benefit of examining the snapshot output file is to learn the physical behavior of the antenna in a seaway. In addition, the output is useful to detect when the time step size is too small, which is characterized by rapid unphysical, antenna oscillations. matlab_output=.TRUE. means that snapshot output is enabled, .FALSE. means snapshot output is disabled.

\section{Simulation Parameters and Results Summary Option (run_info)}

The simulation parameters and results summary file (run_id//'_info.txt') is optional, but is highly recommended as it contains all of the pertinent inputs and results of the simulation. run_info=.TRUE. means that summary output is enabled, .FALSE. means summary output is disabled.

\section{Exposure Data Matrix Option (exp_data)}

The exposure data matrix file (run_id//'_edm') is optional; since, it is needed only when examining the exposure of individual elements rather than being concerned with the overall exposure of the antenna. exp_data=.TRUE. means that the exposure data matrix output is enabled, .FALSE. means that the exposure data matrix output is disabled.

\section{Temporal Wave Data Option (wave_temporal)}

The temporal wave data file (run_id//'_mat2') is optional, since it is needed only when analyzing the power spectrum of the modeled seas in time. It contains the wave elevation at a single random point on the antenna in time. wave_temporal=.TRUE. means that the temporal wave data output is enabled, .FALSE. means that the temporal wave data output is disabled.

\section{Spatial Wave Data Option (wave_spatial)}

The spatial wave data file (run_id//'_mat3') is optional, since it is needed only when analyzing the power spectrum of modeled seas along the antenna. It contains the wave elevation at every point on the antenna for the time $=0.0$ seconds. wave_spatial=.TRUE. means that the spatial wave data output is enabled, .FALSE. means that the spatial wave data output is disabled. 


\section{Significant Wave Height (sig_wave_ht)}

This variable sets the sea's significant wave height, a parameter used to describe the sea severity in both the Bretschneider and Ochi spectral models." Appendix B correlates sea state to significant wave height for a fully developed sea.

\section{Development (development)}

This variable allows the user to shift the sea spectral energy modal frequency up, for developing seas, or down, for decaying seas. This parameter applies only to the Bretschneider spectral model.

\section{Spectral Model (spectral_model)}

Four spectral model subroutines are available: the two parameter Bretschneider spectrum, the Ochi "most probable" six parameter spectrum, a square wave spectrum, and a single sine wave spectrum. Option'1' means Bretschneider, '2' means Ochi, '3' means square, and '4' means sinusoidal. The square wave spectrum and the sine wave spectrum were included for debugging and validation of the results. Parameters of the square wave spectrum cannot be changed with the input file, but the sine wave amplitude and frequency are computed from the sig_wave_ht and development variables. The user is directed to review the source code for the sine wave subroutine to determine how these two input variables control the sine wave parameters.

Selection of the spectral model has a measurable impact upon the exposure statistics computed by the code. There is no litmus test that the user can apply in selecting the correct spectral model, but the different models are better suited for some specific conditions. The assumptions used in deriving the Bretschneider spectrum from North Atlantic sea data were deep water, unlimited fetch, uni-directional seas, and no swell. The Ochi spectrum was derived using the same assumptions except that it allows for swell from a distant storm. Based on those assumptions, one can see that the Bretschneider spectrum is more suited for open ocean wind driven seas, and the Ochi spectrum is more suited for open ocean wind and swell driven seas. 


\section{Directional Spreading Function (spreading_func)}

This allows the user to select the directional spreading function to apply to the power spectra to obtain the desired wave field. Two directional spreading functions are available: $\cos ^{2} \theta$ and $\cos ^{4} \theta$. Option ' 1 ' means $\cos ^{2} \theta$ and option '2' means $\cos ^{4} \theta$.

\section{Number of Propagation Directions $(N N)$}

This allows the user to select the number of discrete unidirectional waves which will combine to form the multidirectional sea surface. A larger number of directions will result in more realistic sea spectra but at the expense of computation time. It is recommended that the user experiment with the number of propagation directions until the simulation results converge.

\section{Trapezoidal Integration Convergence Factor (epsilon)}

This refers to the convergence factor of the trapezoidal integration that is needed to calculate the integral of the directional spreading function. A typical value is $1 e^{-3}$.

\section{Antenna Tow Angle (tow_angle)}

Antenna tow angle with respect to the primary axis of propagation. A tow angle of 0 degrees represents following seas, 90 degrees represents beam seas, and 180 degrees represents head seas.

\section{Antenna Diameter (diam_antenna)}

This is the outer diameter of the hose-like structure encasing the antenna. Element performance (gain) is controlled by whether this outer diameter is submerged; the diameter of the inner conducting slot antenna affects the rotational invariance of the element, and the maximum theoretical gain of a fully exposed element.

\section{Water Density (rho_water)}

Water density in which the antenna operates. 


\section{Drogue Size $\left(\right.$ drog_area $_{\text {_ }}$}

The drogue frontal area (in $\mathrm{m}^{2}$ ) determines the tension at the trailing end of the antenna, according to

$$
T=\frac{1}{2} \rho_{\text {water }} U^{2} d r o g \_ \text {area } C D \_n
$$

A typical value is $0.01 \mathrm{~m}^{2}$.

\section{Normal Drag Coefficient $\left(C D_{-} n\right)$}

This represents the drag coefficient for a cylinder in cross flow. A constant value of 1.0 has been assumed.

\section{Tangential Drag Coefficient $\left(C D_{-} t\right)$}

This is the skin friction associated with flow along the long axis of the antenna. A relatively smooth outer surface has been assumed in choosing this value, which has been assumed constant. A typical value is 0.0035 .

\section{Antenna Density (rho_antenna)}

The average antenna density, which is assumed to be constant over the entire array. This is an approximation in that the antenna has slightly higher density at the actual element, and slightly lower density between elements.

\section{Antenna Lateral Stiffness (flex_stiff_coeff)}

This variable represents the resistance of the antenna to bending, and is given by

$$
\text { flex_stiff_coeff }=E I
$$

where

$E$ is the modulus of elasticity

$I$ is the area moment of inertia. 


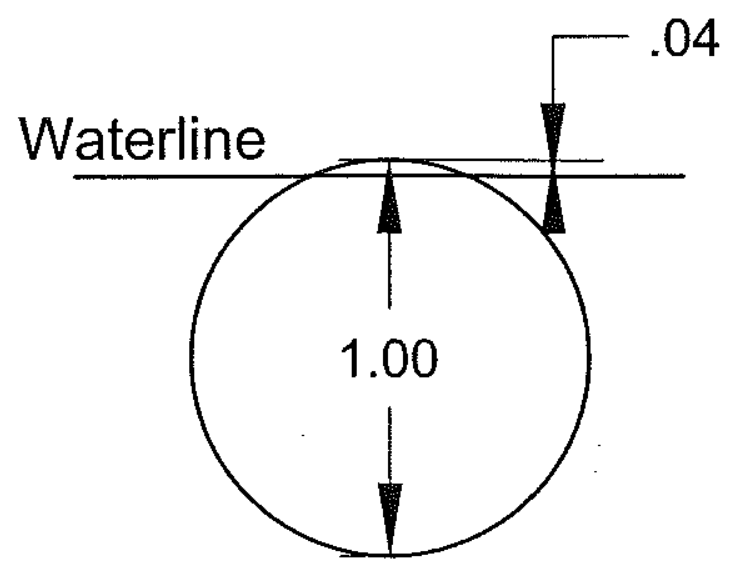

Figure A-1: Sample Threshold Antenna Exposure of 0.04

\section{Added Mass Coefficient $\left(C_{-} m\right)$}

The added mass coefficient for a cylinder in cross flow has been set to 1.0.

\section{Maximum Number of Samples (max_num_of_samples)}

This refers to the maximum number of "snapshots" taken for the Matlab ${ }^{\circledR}$ viewer, or the exposure data matrix. max_num_of_samples is used to dynamically allocate array storage for variables which are sampled periodically in time.

\section{Threshold Antenna Exposure (reqd_ant_exposure)}

As mentioned earlier, the antenna gain remains steady until the element just becomes submerged. In order to capture this performance, and introduce margin for capillary waves not modeled, the parameter reqd_ant_exposure has been defined. The reqd_ant_exposure is the height above the water which must be maintained by the antenna to be considered exposed. The reqd_ant_exposure is given as a fraction of the antenna diameter. See Figure A-1 for a diagram representing an antenna diameter of 1.0 and a reqd_ant_exposure of 0.04 . 


\section{Threshold Number of Elements (num_of_elem_thresh)}

This variable allows the user to specify the lowest number of exposed antenna elements necessary to achieve the desired design antenna array gain. At each stat_time, the number of exposed elements is compared to num_of_elem_thresh; the result (a "1" when the threshold is met, " 0 " otherwise) is found in the summary output file, run_id//'_info.txt', along with any other variables tracked over the course of the simulation. Currently, the variables tracked over time are

- tow point tension (tow_tension ( ))

- number of elements exposed (is_exposed $()$,

- threshold met (thresh_met: "0" or "1", as above)

- counterdetection length (length_totally_exposed( )). 


\section{A.2.2 Sample Input File}

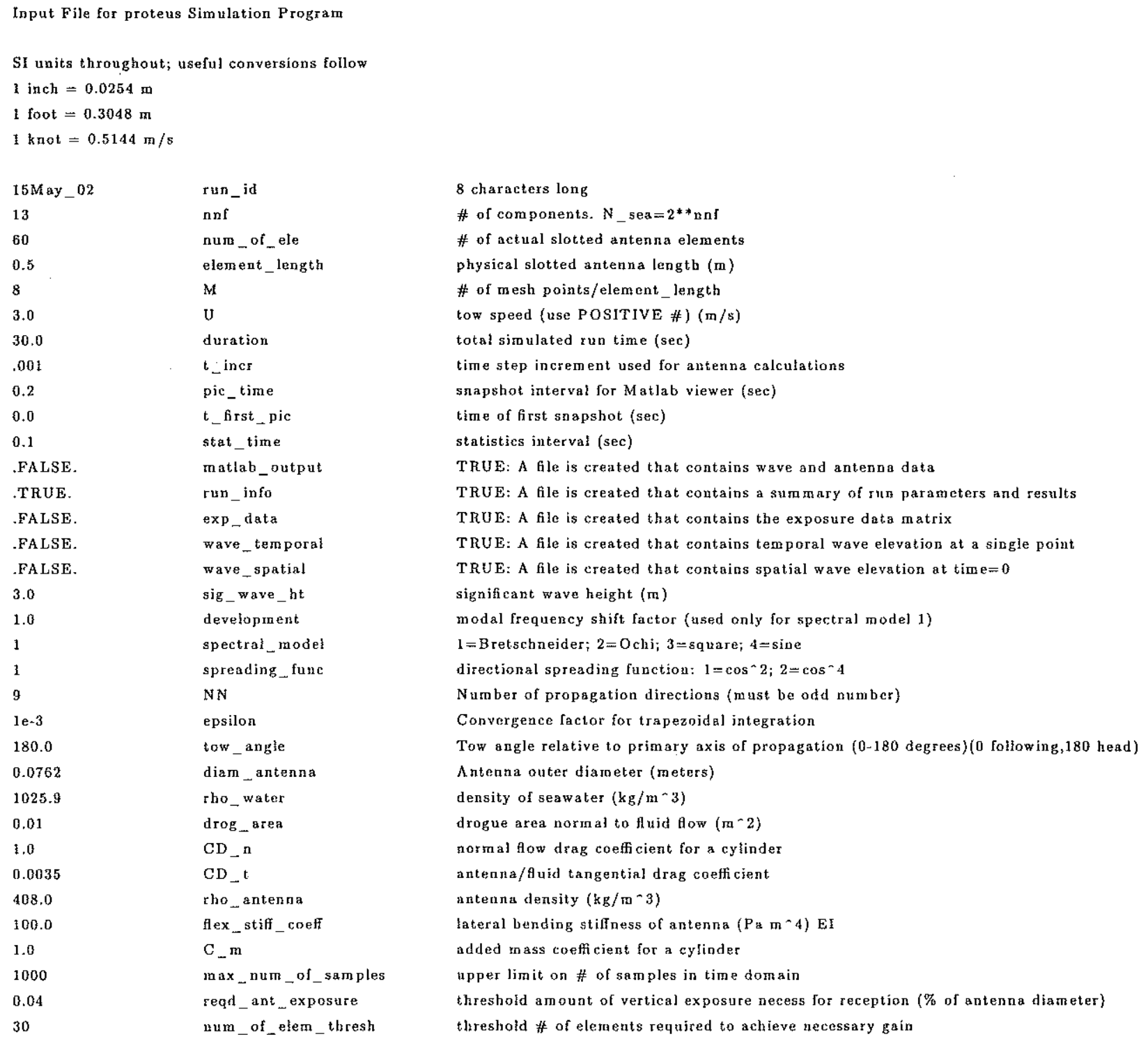

\section{A.3 Output Files}

\section{A.3.1 Snapshot Output File (run_id//'_mat')}

\section{File Description}

This file provides: 


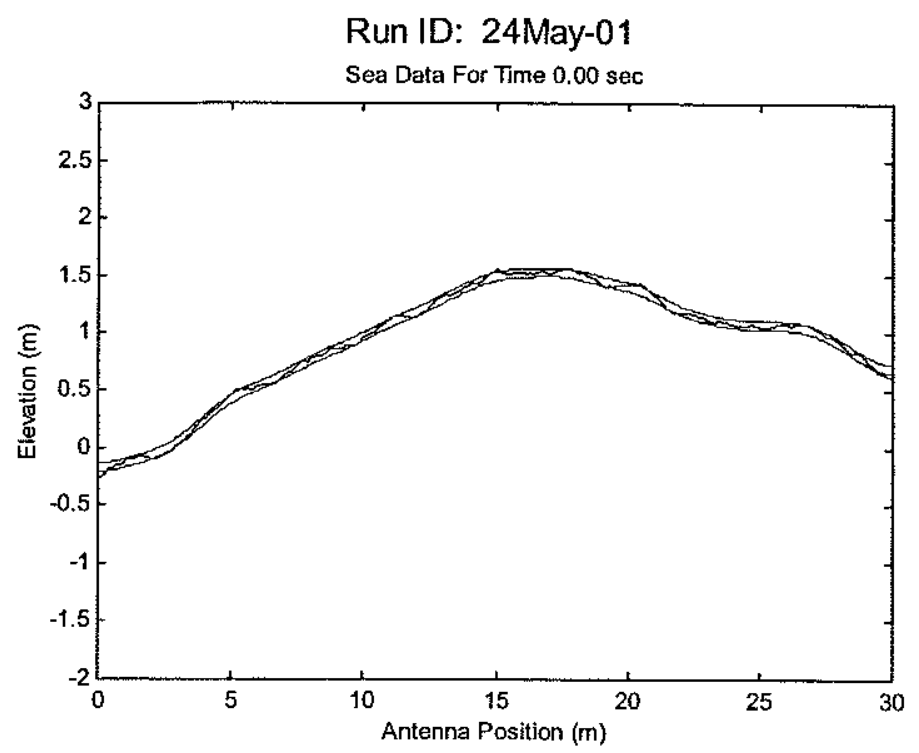

Figure A-2: Sample Output of Snapshot Output File

- a time history of sea elevation along the antenna

- a time history of vertical, longitudinal, and transverse sea velocities along the antenna

- a time history of vertical antenna position

- antenna parameters necessary for the Matlab ${ }^{\circledR}$ function file.

This file is intended to be viewed with the Matlab ${ }^{\circledR}$ function file, proteus_mat.

\section{Sample Output}

A sample output of the Matlab ${ }^{\circledR}$ function file proteus_mat is shown in Figure A-2

\section{A.3.2 Simulation Parameters and Results File (run_id//'_info.txt')}

\section{File Description}

This file provides:

- a complete listing of antenna parameters 
- a complete listing sea spectra parameters

- a summary of exposure and antenna performance statistics.

This file is intended to be viewed with any standard text editor.

\section{Sample Output}

Summary of Parameters and Results

General Information

$---\cdots-\cdots---$

Run ID $=24 \mathrm{May}_{-} 01$

Parameter and Result Summary File $=24 \mathrm{M}_{\text {ay }} 01_{-}$info.txt

Wave and Antena Data File $=24 \mathrm{May}_{-} 01_{-}$mat

Start Date/Time $=05 / 12 / 200007: 22: 31$

End Date/Time $=05 / 12 / 200007: 41: 07$

Ejapsed Computational Time $=1115.56$ seconds

Simulated Analysis Time $=30.00$ seconds

Antenna Parameters

Number of Antensa Elements $=60$

Length of Antenna Element $=0.50$ meters

Total Antenna Length $=30.00$ meters

Antenna Diameter $=0.0762$ meters

Lateral Bending Stiff ness $=50.00 \mathrm{~N}-\mathrm{m}^{\wedge} 2$

Anterna Density $=408.00 \mathrm{~kg} / \mathrm{m}^{-3}$

Drogue Area Normal to Fluid Flow $=0.0100 \mathrm{~m}^{\wedge} 2$

Normal Flow Drag Coeflicient for a Cylinder $=1.000000$

Tangential Drag Coefficient $=0.003500$

Coefficient of Azded Mass for a Cylinder $=1.000000$ 
Number of Mesh Points Per Element $=8$

Tow Speed $=2.50 \mathrm{sm} / \mathrm{sec}$

Tow Angle $=180.0$ degrees

Time Increment for Antenat Related Calculations $=0.00010$ seconds

Sea Spectra Parameters

Spectral Model $=$ Bretschneider

Directional Spreading Function $=\cos ^{\wedge} 2$

Development Factor $=1.00$

Significant Wave Height $=3.00$ meters

Minimum Cutof Frequency $=0.35 \mathrm{radians} / \mathrm{sec}$

Maximum Cutoff Frequency $=22.21 \mathrm{radians} / \mathrm{sec}$

Length of Seas Modeled $=512.0$ meters

Number of Spatial Sea Domain Mesh Points $=2 \backsim 13$

Number of Propagation Directions $=3$

Seawater Density $=1025.90 \mathrm{~kg} / \mathrm{m}^{\wedge} 3$

Time Increment Ior Sea Spectra Related Calculations $=0.02125$ seconds

Number of Antema Time Increments Between Sea Spectra Calculations $=212$

Simulation Results

Maximum Tow Tension $=116.4 \mathrm{~N}$

Minimum Tow Tension $=24.3 \mathrm{~N}$

Stretched Antenna Length $=30.2$ meters

Antenna Strain $=.005$

Statistics Sampling Interval $=0.100$ seconds

Number of Samples Observed for Statistics $=301$

Average Fraction of Antenna Exposed $=0.895$

Vertical Antenna Exposure Necessary to Receive $=4.0$ percent of diameter 
Number of Antenna Elements Required to be Exposed $=30 / 60$

Time That Number of Required Elements are Exposed $=100.0$ percent

Average Detection Length $=1.95$ meters

\section{A.3.3 Exposure Data Matrix File (run_id//'_edm')}

\section{File Description}

This file provides:

- the raw exposure data information for each antenna element at each time sampled

- the spatial position of all elements.

This allows one to analyze statistics across time or space. This file can be viewed with a user supplied Matlab ${ }^{(\mathbb{R})}$ file or with a spreadsheet application.

\section{Sample Output}

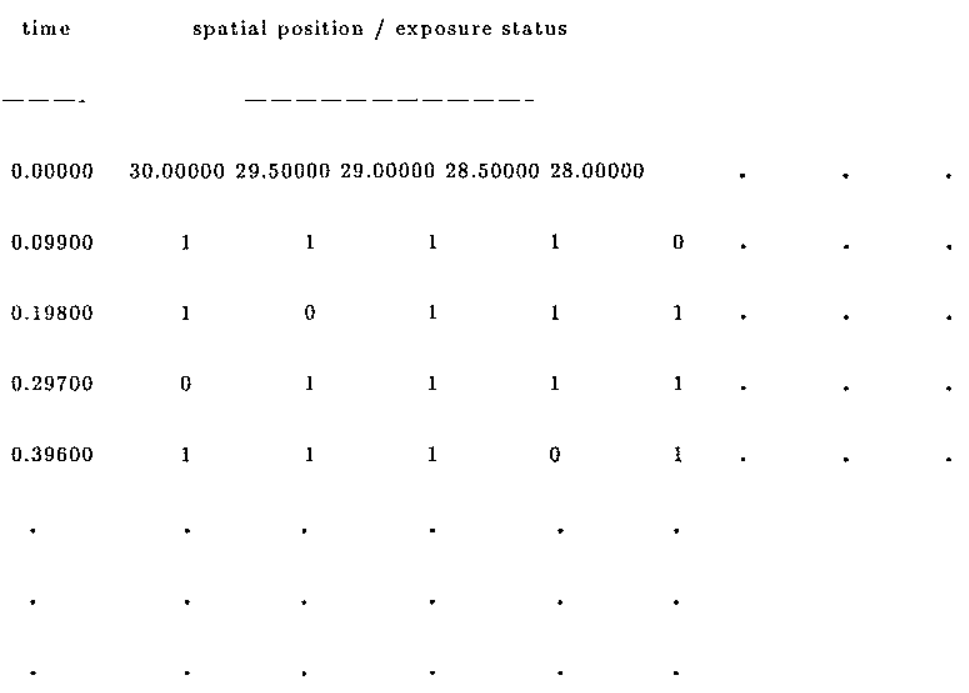

\section{A.3.4 Temporal Wave Data File ( $r u n_{-} i d / /$ '_mat2')}

\section{File Description}

This file provides: 


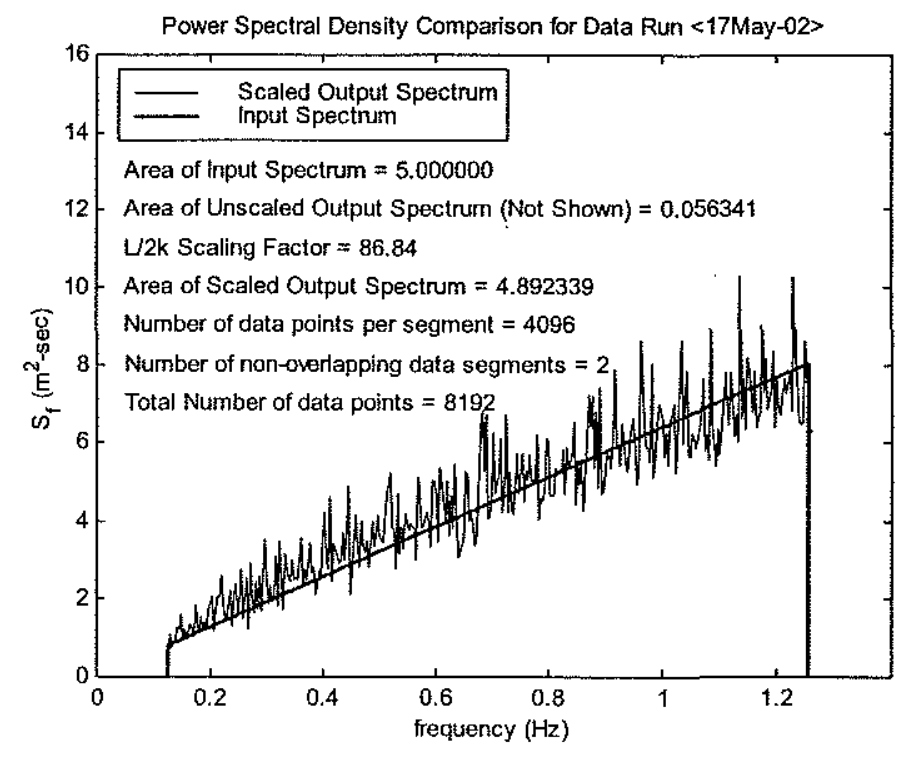

Figure A-3: Sample Output of Temporal Wave Data Analysis

- a time history of the wave elevation at a single random point on the antenna

- antenna parameters necessary for the Matlab ${ }^{\circledR}$ function file.

This allows one to analyze the power spectrum of the modeled seas in time. It is intended to be processed with the FORTRAN executable file, proteus_mat2.exe, then viewed with the Matlab ${ }^{\circledR}$ function file, proteus_mat2.

\section{Sample Output}

A sample output of the Matlab ${ }^{\circledR}$ function file proteus_mat2 is shown in Figure A-3.

\section{A.3.5 Spatial Wave Data File (run_id//'_mat3')}

\section{File Description}

This file provides:

- the wave elevation at every point on the antenna at time $=0.0$ seconds

- antenna parameters necessary for the Matlab ${ }^{\circledR}$ function file. 


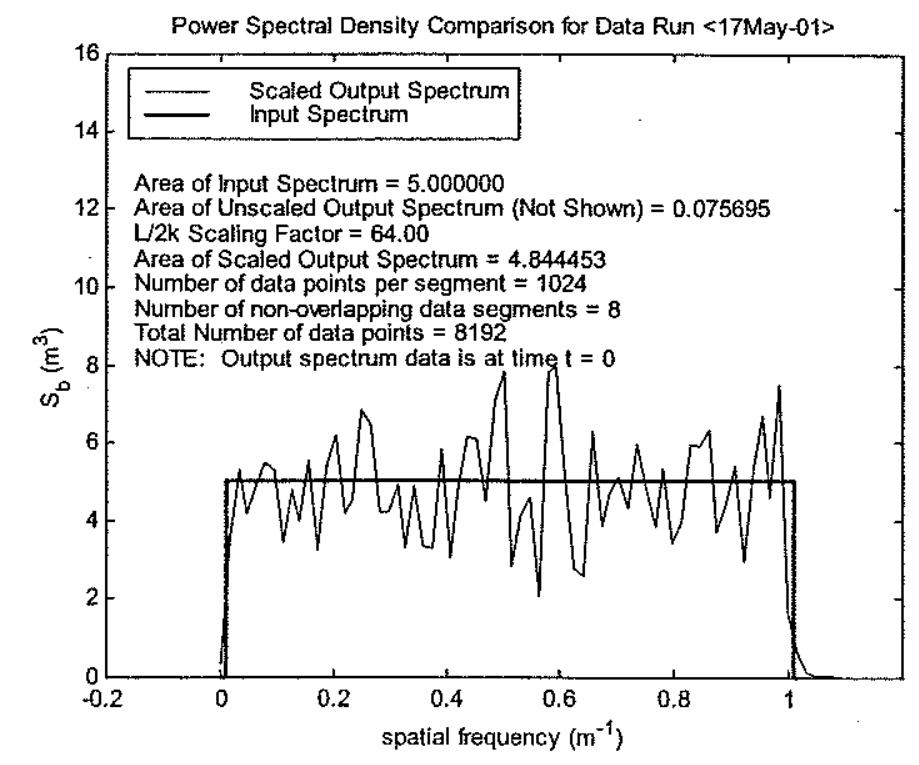

Figure A-4: Sample Output of Spatial Wave Data Analysis

This allows one to analyze the power spectrum of the modeled seas in space. It is intended to be processed with the FORTRAN executable file, proteus_mat3:exe, then viewed with the Matlab $^{\circledR}$ function file, proteus_mat?.

\section{Sample Output}

A sample output of the Matlab ${ }^{\circledR}$ function file proteus_mat3 is shown in Figure A-4. 
Appendix B

Sea State Correlation Data 


\begin{tabular}{|c|c|c|c|}
\hline $\begin{array}{c}\text { Significant } \\
\text { Wave Height } \\
(\mathrm{m}) \\
\end{array}$ & $\begin{array}{c}\text { Modal } \\
\text { Frequency } \\
(\mathrm{rad} / \mathrm{sec})\end{array}$ & $\begin{array}{c}\text { Wind } \\
\text { Speed } \\
\text { (knots) }\end{array}$ & $\begin{array}{c}\text { Sea } \\
\text { State }\end{array}$ \\
\hline 0.1 & 3.96 & 4.2 & \\
\hline 0.2 & 2.80 & 5.9 & \\
\hline 0.3 & 2.29 & 7.3 & 1 \\
\hline 0.4 & 1.98 & 8.4 & \\
\hline 0.5 & 1.77 & 9.4 & \\
\hline 0.6 & 1.62 & 10.3 & \\
\hline 0.7 & 1.50 & 11.1 & \\
\hline 0.8 & 1.40 & 11.9 & 2 \\
\hline 0.9 & 1.32 & 12.6 & \\
\hline 1.0 & 1.25 & 13.3 & \\
\hline 1.1 & 1.20 & 13.9 & \\
\hline 1.2 & 1.14 & 14.6 & \\
\hline 1.3 & 1.10 & 15.2 & 3 \\
\hline 1.4 & 1.06 & 15.7 & \\
\hline 1.5 & 1.02 & 16.3 & \\
\hline 1.6 & 0.99 & 16.8 & \\
\hline 1.7 & 0.96 & 17.3 & \\
\hline 1.8 & 0.93 & 17.8 & \\
\hline 1.9 & 0.91 & 18.3 & \\
\hline 2.0 & 0.89 & 18.8 & 4 \\
\hline 2.2 & 0.85 & 19.7 & \\
\hline 2.4 & 0.81 & 20.6 & \\
\hline 2.6 & 0.78 & 21.4 & \\
\hline 2.8 & 0.75 & 22.2 & \\
\hline 3.0 & 0.72 & 23.0 & 5 \\
\hline 3.2 & 0.70 & 23.8 & \\
\hline 3.4 & 0.68 & 24.5 & \\
\hline 3.6 & 0.66 & 25.2 & 6 \\
\hline 3.8 & 0.64 & 25.9 & \\
\hline 4.0 & 0.63 & 26.6 & \\
\hline 4.5 & 0.59 & 28.2 & \\
\hline 5.0 & 0.56 & 29.7 & \\
\hline 5.5 & 0.53 & 31.2 & \\
\hline 6.0 & 0.51 & 32.6 & \\
\hline 6.5 & 0.49 & 33.9 & 7 \\
\hline 7.0 & 0.47 & 35.2 & \\
\hline 7.5 & 0.46 & 36.4 & \\
\hline 8.0 & 0.44 & 37.6 & \\
\hline 8.5 & 0.43 & 38.8 & \\
\hline 9.0 & 0.42 & 39.9 & \\
\hline
\end{tabular}

Table B.1: Sea State Correlation Data 


\section{Bibliography}

[1] Berteaux, H. O. Buoy Engineering. New York: John Wiley and Sons, Inc., 1976.

[2] Celia, M. A. and W. G. Gray. Numerical Methods for Differential Equations. Englewood Cliffs, NewJersey: Prentice Hall, 1992.

[3] Chapra, Steven C. and Raymond P. Canale. Numerical Methods for Engineers (Second Edition). New York: McGraw-Hill Publishing Company, 1988.

[4] Faltinsen, O. M. Sea Loads on Ships and Offshore Structures. Cambridge Ocean Technology Series - Volume One, United Kingdom: Cambridge University Press, 1990.

[5] Massel, Stanislaw R. Ocean Surface Waves: Their Physics and Prediction. Advanced Series on Ocean Engineering - Volume Eleven, Singapore: World Scientific Publishing, 1996.

[6] Newman, John N. Marine Hydrodynamics. Cambridge, Massachusetts: MIT Press, 1977.

[7] Ochi, Michel K. Ocean Waves. Cambridge Ocean Technology Series - Volume Six, United Kingdom: Cambridge University Press, 1998.

[8] Triantafyllou, M.S. and C. Chryssostomidis. Environment Description, Force Prediction and Statistics for Design Applications in Ocean Engineering. Cambridge, MA: Department of Ocean Engineering, Massachusetts Institute of Technology, 1980.

[9] Ulrich, Gary A., "Computer Model for a Towed Submarine Communication Antenna." Naval Engineer thesis, Massachusetts Institute of Technology, Cambridge, Massachusetts, 1999. 